\title{
Quantum Affine Algebras and Holonomic Difference Equations
}

\author{
I. B. Frenkel ${ }^{1}$ and N. Yu. Reshetikhin ${ }^{2}$ \\ 1 Department of Mathematics, Yale University, New Haven, CT 06520, USA \\ 2 Department of Mathematics, University of California at Berkeley, Berkeley, CA 94720, USA
}

Received September 19, 1991; in revised form November 15, 1991

\begin{abstract}
We derive new holonomic $q$-difference equations for the matrix coefficients of the products of intertwining operators for quantum affine algebra $U_{q}(\hat{\mathfrak{g}})$ representations of level $k$. We study the connection opertors between the solutions with different asymptotics and show that they are given by products of elliptic theta functions. We prove that the connection operators automatically provide elliptic solutions of YangBaxter equations in the "face" formulation for any type of Lie algebra $\mathfrak{g}$ and arbitrary finite-dimensional representations of $U_{q}(\hat{\mathfrak{g}})$. We conjecture that these solutions of the Yang-Baxter equations cover all elliptic solutions known in the contexts of IRF models of statistical mechanics. We also conjecture that in a special limit when $q \rightarrow 1$ these solutions degenerate again into $U_{q^{\prime}}(\hat{\mathfrak{g}})$ solutions with $q^{\prime}=\exp \left(\frac{2 \pi i}{k+g}\right)$. We also study the simplest examples of solutions of our holonomic difference equations associated to $U_{q}(\widehat{\mathfrak{s l}(2)})$ and find their expressions in terms of basic (or $q-$ )-hypergeometric series. In the special case of spin $-\frac{1}{2}$ representations, we demonstrate that the connection matrix yields a famous Baxter solution of the Yang-Baxter equation corresponding to the solid-on-solid model of statistical mechanics.
\end{abstract}

\section{Introduction}

The recent development in mathematics and physics related to conformal field theory [BPZ, FS, S, MS] and quantum groups [Kr, Dr1, J2] is a result of an astonishing interplay between various ideas of both sciences (see [0] for a partial bibliography). Mathematical roots of these theories lie in the representation theory of infinite dimensional Lie algebras and groups, algebraic geometry and Hamiltonian mechanics. The physical intuition arises from quantum field theory in two dimensions, integrable models in statistical mechanics and string theory. For mathematicians conformal field theory is a representation of certain geometric categories of Riemann Surfaces [S] or a regular representation of a "Lie algebra depending on a parameter" (vertex operator algebra) [FLM, MS]. For physicists, it is first of all the theory that characterizes the 
critical behavior of two dimensional physical systems; another fundamental role of conformal field theory is that it describes the classical limit of string theory. Presently, the general picture of conformal field theory is well understood from both mathematical and physical points of view and one can wonder about its further generalizations. Here the different approaches suggest its own program of research (see [1-3] for examples of several directions). In this paper we propose a few steps of an extension of conformal field theory using the ideas of the representation theory [1]). However, as a result of our work, we unavoidably arrive at new connections with the other areas of mathematics and physics [2,3], which were previously essentially unrelated. Thus our work can also be thought of as another contribution to the remarkable synthesis that takes place in mathematics and physics.

One of the most fundamental examples of conformal field theory is the WessZumino-Novikov-Witten model (WZNW) [W1]. It is based on the representation theory of affine Lie algebras (or loop algebras) and the corresponding groups [Koh, PS]. In particular, the genus zero correlation functions of WZNW model are the matrix coefficients of intertwining operators between certain representations of affine Lie algebras $[\mathrm{TK}]$. The monodromy properties of the correlation functions contain the most essential structural information about specific conformal field theory. Thus the algebra of intertwining operators present a special interest for the WZNW model. One way to study this algebra is to show that the matrix coefficients of the intertwining operators satisfy certain holonomic differential equations first derived by Knizhnik and Zamolodchikov [KZ]. Since the simplest nontrivial examples appear to be differential equations for the hypergeometric function and its classical generalizations, the theory of Knizhnik-Zamolodchikov equations can be thought of as a far-reaching extension of the theory of hypergeometric functions. In fact, one can view the Knizhnik-Zamolodchikov equation as a connection on certain flat vector bundles on $\mathbf{P}^{1}$ and more generally on an arbitrary Riemann surface and then proceed to study their structure by the methods of algebraic geometry. The relation of the representation theory and algebraic geometry via the Knizhnik-Zamolodchikov equation has a deep parallel in the quantum field theory in the Wightman program relating the algebraic structure of the Hilbert space of states to the properties of the correlation functions. The theory of Knizhnik-Zamolodchikov equation thus provides a perfect example of the realization of this program.

The most substantial examples of quantum groups are certain $q$-deformations of the linear space of regular functions on a simple Lie group $G$. Its dual algebra $U_{q}(\mathfrak{g})$ is naturally identified with a $q$-deformation of the universal enveloping algebra $U(\mathfrak{g})$ of a simple Lie algebra $\mathfrak{g}$ corresponding to $G$. One can extend the definition of $U_{q}(\mathfrak{g})$ to an arbitrary Kac-Moody algebra, in particular, to the affine Lie algebra $\hat{\mathfrak{g}}$ associated to $\mathfrak{g}$. We will call, for shortness, $U_{q}(\mathfrak{g})$ quantum algebra, and $U_{q}(\hat{\mathfrak{g}})$ quantum affine algebra.

It was gradually realized that the WZNW conformal field theory and the representation theory of quantum groups have a profound link. In particular, the monodromies of the Knizhnik-Zamolodchikov equation are directly related to the intertwining operators for the tensor products of quantum groups [Koh, D2]. Thus the quantum groups can be viewed in some sense as hidden symmetries of conformal field theory [MR]. This amazing relation is still not fully understood and is a subject of an intense study [SV1, SV2, KaL].

Apart from the remarkable but still mysterious relation to the conformal field theory, the theory of quantum groups and quantum algebras can be developed to a great extent parallel to the theory of simple Lie groups and Lie algebras. Practically 
any aspect of the theory of simple and affine Lie algebras admit an appropriate $q$ deformation. To begin with, besides a $q$-analogue of the universal enveloping algebra, there exists a corresponding deformation of the highest weight representations [L, RO1]. However, the correct quantum analogues are not always straightforward and one should expect to encounter radically new phenomena. An important program for future research is a $q$-deformation of the entire structure associated to conformal field theroy. Only a few isolated results in this direction are presently known [FJ, BL]. One of the key questions towards realization of this program is to find an analogue of the Knizhnik-Zamolodchikov equation, study its solutions and identify its hidden symmetries. We address these problems in the present paper.

Our main result is a derivation of an analogue of the Knizhnik-Zamolodchikov equation for quantum affine algebras. The new equation appears to be a certain linear $q$-difference equation satisfying holonomy conditions. As in the corresponding case of conformal field theory, this equation is deduced for the matrix coefficients of a product of intertwining operators. Our derivation uses the multiplicative approach to quantum affine algebras [RS], which we develop further in the earlier sections. Our next result concerns the properties of connection matrices for the solutions with different asymptotics. These matrices, which play the role of monodromies in the conformal field theory case, are not constant in the quantum case but they depend on a spectral parameter. Using a classical result of Birkhoff [Bi] from the theory of $q$-difference equations, we show that matrix coefficients of connection matrices can be expressed in terms of ratios of elliptic theta functions, or in other words, sections of a line bundle on an elliptic curve. We show that connection matrices satisfy a version of the Yang-Baxter equations known as star-triangle relations in accord with the terminology of the book [B2].

For quantum $\widehat{\mathfrak{s l}(2)}$ we find an explicit expression of solutions of our $q$-difference equations in terms of basic (or $q$-)hypergeometric functions introduced in the last century $[\mathrm{H} 1, \mathrm{H} 2]$, and we compute explicitly the connection matrix and identified it with the Baxter solution of the star-triangle relation for the solid-on-solid model [B1].

Our results have two immediate implications, one in mathematics and another in physics. We mentioned before the relation between monodromies of the KnizhnikZamolodchikov equation and quantum algebras $U_{q}(\mathfrak{g})$ [Koh, D2, SV1, SV2]. This relation alone involves a substantial number of different mathematical structures. In this paper we conjecture that a similar relation exists between the trigonometric limit (see below) of connection matrices and finite-dimensional representations of quantum affine algebras $U_{q^{\prime}}(\hat{\mathfrak{g}})$ with $q^{\prime}=\exp \left(\frac{2 \pi i}{k+g}\right)$, which now plays the role of hidden symmetries. Since this correspondence reduces to the previous one when the spectral parameter tends to infinity, one can expect a new level of mathematical structures. The elliptic case is even more interesting. The algebras that describe the hidden symmetries of our $q$-difference equation have not been defined yet. They must be further deformations of $U_{q^{\prime}}(\hat{\mathfrak{g}})$, which yield elliptic solutions of Yang-Baxter equations as intertwining operators. Since the solutions of $q$-difference equations are given by generalized basic hypergeometric functions, one can expect that the representation theory will allow to understand the conceptual meaning behind numerous remarkable identities in this chapter of mathematics [S1, GR], and will suggest far-reaching generalizations.

The physical implication of our results concerns integrable models in statistical physics. There exist extensive generalizations of the original Baxter solution of the 
star-triangle relation for other types of Lie algebras and various finite-dimensional representations [DJMO, DJKMO, JMO]. We conjecture that all these solutions come from the connection matrices of our $q$-difference equations. The star-triangle relation is only the very first fundamental aspect of integrable models in statistical mechanics. We expect that many other physical concepts will also find their place and explanation in the representation theory of quantum affine algebras.

There is another remarkable relation with physics. When the central element in $\hat{\mathfrak{g}}$ acts by zero our $q$-difference equation coincides with one of Smirnov's equations for form factors in integrable two-dimensional models derived from basic principles of quantum field theory and the factorizability of the $S$-matrix a few years ago [Sm1]. This relation connects in a conceptual way deformations of universal enveloping algebras of affine Lie algebras and massive integrable deformations of conformal field theory [Z]. These models might be another class of examples where Wightman's program can be explicitly realized. We also believe that interpretation of form factors as deformed Knizhnik-Zamolodchikov equations will help to understand infinite dynamical symmetries of integrable models [Be, $\mathrm{LSm}$ ] and may lead to interesting new aspects of representation theory of infinite dimensional algebras.

We will delay further discussion of the future problems and perspectives to the conclusion and will turn to the more technical description of our results.

In Sect. 2 we recall the derivation of the Knizhnik-Zamolodchikov equations in the form convenient for our generalizations. To any irreducible finite dimensional representation $V_{\lambda}$ of a simple Lie algebra $\mathfrak{g}$ indexed by a highest weight $\lambda$ one can associate two types of representations of the corresponding affine Lie algebra $\hat{\mathfrak{g}}$. One type is again the highest weight representation $V_{\lambda, k}$ of level $k$ (equal to the value of the central element $c$ ). This representation has a $\mathbf{Z}$-graded structure $V_{\lambda, k}=\bigoplus_{n \in \mathbf{Z}_{+}} V_{\lambda, k}[-n]$ compatible with the one of $\hat{\mathfrak{g}}$ and its top subspace $V_{\lambda, k}[0]$ is naturally isomorphic to $V_{\lambda}$. The second type is just a finite dimensional evaluation representation $V_{\lambda}(z)$, $z \in \mathbf{C} \backslash 0$, isomorphic to $V_{\lambda}$ as a vector space for which $k=0$. The operators of the central interest to the conformal field theory are the intertwining operators $\Phi(z)$, between $V_{\lambda, k}$ and $V_{\nu, k} \otimes V_{\mu}(z)$. To formulate the properties of $\Phi(z)$ it is convenient to introduce a generating function $J(z)$ for $\hat{\mathfrak{g}}$ acting naturally on $V_{\nu, k} \otimes V_{\mu}$. Let $\left\{J_{a}\right\}$ be an orthonormal basis of $\mathfrak{g}$ with respect to an invariant form normalized by the condition that the square norm of the highest root is 2 , and let $\left\{J_{a}[n] ; c\right\}, n \in \mathbf{Z}$, be the corresponding basis of $\hat{\mathfrak{g}}$. Then we define $J(z)=\sum_{a, n} J_{a}[n] \otimes J_{a} z^{-n-1}$ and the commutation relations of $\hat{\mathfrak{g}}$ can be written solely in terms of this generating function.

We deduce from the intertwining property of $\Phi(z)$ that under the natural normalization it satisfies an operator of the linear differential equation

$$
(k+g) \frac{d}{d z} \Phi(z)=: J(z) \Phi(z):
$$

where $g$ is the dual Coxeter number and :: is a normal ordering of operators defined by means of the decomposition of $J(z)$ into the sum of "analytic" and "antianalytic" parts (2.12).

The operator linear differential equation (1.1) immediately implies that the matrix coefficients of the product of $\Phi$ 's

$$
\Psi=\left\langle v_{0}, \Phi_{1}\left(z_{1}\right) \ldots \Phi_{N}\left(z_{N}\right) v_{N+1}\right\rangle \in V_{1} \otimes \cdots \otimes V_{N}
$$


where $v_{0}$ belongs to $V_{\lambda, k}[0]$ and $v_{N+1}$ is the highest weight vector in $V_{0, k}$ satisfies the Knizhnik-Zamolodchikov equation

$$
(k+g) \frac{\partial \Psi}{\partial z_{i}}=\sum_{j \neq i} \frac{\Omega_{i j}}{z_{i}-z_{j}} \Psi,
$$

where $\Omega_{i j}=\pi_{i}\left(J_{a}\right) \otimes \pi_{j}\left(J_{a}\right)$ and $\pi_{i}$ is the representation in $V_{i}$. Equation (1.3) is a holonomic differential equation and defines a flat vector bundle over $\mathbf{P}^{1} \backslash\left\{z_{1}, \ldots, z_{N}\right\}$.

The holonomic property of the Knizhnik-Zamolodchikov equations is tied to the fact that $r_{i j}\left(z_{i}-z_{j}\right)=\frac{\Omega_{i j}}{z_{i}-z_{j}}$ is a solution of the classical Yang-Baxter equation

$$
\begin{aligned}
{\left[r_{12}\left(z_{1}-z_{2}\right), r_{13}\left(z_{1}-z_{3}\right)\right]+} & {\left[r_{12}\left(z_{1}-z_{2}\right), r_{23}\left(z_{2}-z_{3}\right)\right] } \\
+ & {\left[r_{13}\left(z_{1}-z_{3}\right), r_{23}\left(z_{2}-z_{3}\right)\right]=0 . }
\end{aligned}
$$

We show how to transform the Knizhnik-Zamolodchikov equation to the "trigonometric" form with $r_{i j}\left(z_{i}-z_{j}\right)$ replaced by a trigonometric solution of the Yang-Baxter equation $\tilde{r}_{i j}\left(z_{i} / z_{j}\right)$. Choosing $v_{0}$ and $v_{N+1}$ to be lowest and highest weight vectors and multiplying $\Psi$ by apropriate powers of $z_{i}$ 's (denoted $\tilde{\Psi}$ ) one obtains

$$
(k+g) z_{i} \frac{\partial \tilde{\Psi}}{\partial z_{i}}=\left\{\sum_{j \neq i} \tilde{r}_{i j}\left(z_{i} / z_{j}\right)+\pi_{i}(\lambda)\right\} \tilde{\Psi},
$$

where $\lambda=\left(\lambda_{0}+\lambda_{N+1}+2 \varrho\right) / 2$, and $\varrho$ is a half sum of positive roots of $\mathfrak{g}$. Two forms of Knizhnik-Zamolodchikov equations admit two entirely different quantizations, which are now related to rational and trigonometric forms of the quantum Yang-Baxter equation

$$
R_{12}\left(z_{1} z_{2}^{-1}\right) R_{13}\left(z_{1} z_{3}^{-1}\right) R_{23}\left(z_{2} z_{3}^{-1}\right)=R_{23}\left(z_{2} z_{3}^{-1}\right) R_{13}\left(z_{1} z_{3}^{-1}\right) R_{12}\left(z_{1} z_{2}^{-1}\right) \text {. }
$$

In this paper we concentrate on the trigonometric case, which is based on the representation theory of quantum affine algebra $U_{q}(\mathfrak{g})$. The rational case is related to the representation theory of full Yangians (the double of the Yangian [LSm]) and can be obtained as a certain limit of our constructions.

To prepare the necessary tools we first recall the basic facts on the representation theory of quantum algebra $U_{q}(\mathfrak{g})$ in Sect. 3 and then we proceed to the quantum affine algebra $U_{q}(\hat{\mathfrak{g}})$ in Sect. 4.

The main result of Sect. 3 is Theorem 3.2 which will be used in Sect. 5 when we derive the quantum analogue of the Knizhnik-Zamolodchikov equation.

In Sect. 4 we remind basic properties of the algebra $U_{q}(\hat{\mathfrak{g}})$ and their representations. This algebra (as well as $U(\hat{\mathfrak{g}})$ ) has two different classes of irreducible representations: highest weight modules $V_{\lambda, k}$ and finite dimensional modules $V_{\lambda}(z)$. Intertwining operators $R^{V W}(z)$ between two products of finite dimensional modules $V(x z)$ and $W(x)$ are determined by the universal $R$-matrix for $U_{q}(\hat{\mathfrak{g}})$. These intertwiners satisfy the Yang-Baxter equation. We prove that $R^{V W}(z)$ is a meromorphic function of $z$ and that it satisfies the "crossing-symmetry"

$$
\begin{gathered}
\left(\left(\left(R^{V W}(z)^{-1}\right)^{t_{1}}\right)^{-1}\right)^{t_{1}}=\left(\pi_{V}\left(q^{2 \varrho}\right) \otimes 1\right) R^{V W}\left(z q^{2 g}\right)\left(\pi_{V}\left(q^{-2 g}\right) \otimes 1\right), \\
\left(\left(\left(R^{V W}(z)^{-1}\right)^{t_{2}}\right)^{-1}\right)^{t_{2}}=\left(1 \otimes \pi_{W}\left(q^{-2 \varrho}\right)\right) R^{V W}\left(z q^{-2 g}\right)\left(1 \otimes \pi_{W}\left(q^{2 \varrho}\right)\right),
\end{gathered}
$$

and the "unitarity" relations:

$$
R^{V W}(z) R_{21}^{W V}\left(z^{-1}\right)=I_{V \otimes W}
$$


Then we describe "multiplicative realization" of $U_{q}(\hat{\mathfrak{g}})[\mathrm{RS}]$ using the universal $R$ matrix. The multiplicative realization is crucial for our exposition since it allows us to define a quantum analogue $L(z)$ of the current $J(z)$ for affine Lie algebra. We end this section with the definition of intertwiners $\Phi(z)$ parallel to those for $\hat{\mathfrak{g}}$ and obtain their relations with generators in multiplicative realizations.

In Sect. 5 we deduce the operator linear difference equation for the intertwining operator

$$
\Phi\left(z q^{-(k+g)}\right)=\vdots L\left(z q^{-g}\right) \Phi\left(z q^{(k+g)}\right) \vdots
$$

Here the normal ordering $: \vdots$ is defined using the factorization of $L(z)$ into the "analytic" and "antianalytic" parts (see Sect.4). The relation (1.10) is a $q$-analogue of the differential equation (1.1). We define a general matrix coefficient of the product of intertwining operators as

$$
\mathscr{F}=\left\langle\cdot, \Phi_{1}\left(z_{1}\right) \cdots \Phi_{N}\left(z_{N}\right) \cdot\right\rangle \in V_{0} \otimes V_{1} \otimes \cdots V_{N} \otimes\left(V_{N+1}\right)^{*},
$$

where $V_{0}$ and $V_{N+1}$ are the top subspaces of the corresponding infinite dimensional modules.

Equation (1.10) implies the difference equation for the matrix coefficients of the product of the intertwining operators

$$
T_{i} \mathscr{F}=A_{i} \mathscr{F},
$$

where $T_{i} f\left(z_{1}, \ldots, z_{i}, \ldots, z_{N}\right)=f\left(z_{1}, \ldots, p z_{i}, \ldots, z_{N}\right), p=q^{-2(k+g)}$, and

$$
\begin{aligned}
A_{i}(z)= & R_{i i-1}\left(\frac{p z_{i}}{z_{i-1}}\right) \ldots R_{i 1}\left(\frac{p z_{i}}{z_{1}}\right) \ldots \bar{R}_{i 0} \pi_{i}\left(q^{2 \varrho}\right)\left(\bar{R}_{i N}\right)^{-1} \\
& \times R_{i N}\left(\frac{z_{i}}{z_{N}}\right) \ldots R_{i i+1}\left(\frac{z_{i}}{z_{i+1}}\right) .
\end{aligned}
$$

Here $R_{i j}(z) \in \operatorname{End}\left(V_{i} \otimes V_{j}\right)$ are the intertwining operators ( $R$-matrices) corresponding to the pair of finite dimensional $U_{q}(\hat{\mathfrak{g}})$ modules $V_{i}$ and $V_{j}$ and $\bar{R}_{i j}$ are corresponding $U_{q}(\mathfrak{g})$ intertwining operators. Then we show that the Yang-Baxter equation for $R_{i j}(z)$ implies that (1.11) is a holonomic difference system in the sense of [A1]:

$$
\left(T_{i} A_{j}\right) A_{i}=\left(T_{j} A_{i}\right) A_{j} .
$$

We begin Sect. 6 with the study of the properties of solutions of our difference system (1.11). We show that the subspace of $U_{q}(\mathfrak{g})$-invariant solutions to this system is naturally isomorphic to

$$
\mathscr{H}_{V_{0}, V_{N+1}}^{V_{1} \ldots V_{N}}=\operatorname{Inv}_{U_{q}(g)}\left(V_{0} \otimes V_{1} \otimes \cdots \otimes V_{N} \otimes\left(V_{N+1}\right)^{*}\right) .
$$

We show that solutions with different asymptotics can be analytically continued and we define connection matrices between them. The space (1.13) admits a factorization

$$
\bigoplus_{\lambda_{1} \ldots \lambda_{N-1}}\left(\mathscr{H}_{V_{0} V_{\lambda_{1}}}^{V_{1}} \otimes \mathscr{H}_{V_{\lambda_{1}} V_{\lambda_{2}}}^{V_{2}} \otimes \cdots \otimes \mathscr{H}_{V_{\lambda_{N-1}}^{V_{n}} V_{N+1}}^{V_{n}}\right)
$$

which provide corresponding natural choice of basis in (1.13). We describe explicitly the action of the connection matrices in this basis (1.14) and show that they can be expressed in terms of a product of elementary connection matrices arising from the 
difference equations with $N=2$ as in the classical case. We proved that the elementary connection matrices satisfy the Yang-Baxter equation, a "unitary" condition and that their entries are ratios of theta functions. On the basis of these facts we conjecture that the elementary connection matrices of the system (1.11), (1.12) cover all known solutions of the Yang-Baxter equation [DJMO, JMO]. One of the corollaries of these results is that matrix coefficients of the intertwining operators allow an analytic continuation from formal power series to complex values of $z$. The analytic continuation, together with the factorization property of connection matrices, yield an exchange algebra for the intertwining operators (6.40). We also conjecture that in the limit $q \rightarrow 1, z_{i}=q^{x_{i}}$ and $x_{i}$ fixed these connection matrices coincide with the action of $U_{q^{\prime}}(\hat{\mathfrak{g}})$ intertwiners on the $U_{q}(\mathfrak{g})$ invariant subspaces of tensor products of finite dimensional $U_{q^{\prime}}(\hat{\mathfrak{g}})$-modules with $q^{\prime}=\exp \left(\frac{2 \pi i}{k+g}\right)$. This property of connection matrices for the system (1.11), (1.12) can be regarded as a generalization of the correspondence between $U(\hat{\mathfrak{g}})$-modules of level $k$ and the algebra $U_{q^{\prime}}(\mathfrak{g})$ [MR, D1, SV1, SV2, KaL] and others.

In Sect. 7 we consider a special example of solutions of our difference equation when $\mathfrak{g}=\mathfrak{s l}(2)$. It turns out that they are expressed in terms of the basic or $q$ hypergeometric series introduced in the middle of the last century [H1, H2]. We recall some facts of this theory including the integral formulas and the connection formulas. Applications of the connection formulas for basic hypergeometric series allows us to find explicitly the connection matrices in certain special cases. In particular, when $V_{1}$ and $V_{2}$ are both two-dimensional representations of $U_{q}(\widehat{\mathfrak{s l}(2)})$ we obtain

$$
\begin{aligned}
& W^{V_{1} V_{2}(z)}\left[\begin{array}{lll}
K & K \pm 1 & \\
& K \mp 1 & K
\end{array}\right]=1 \\
& W^{V_{1} V_{2}}(z)\left[\begin{array}{rrr}
K \pm 1 & & K \pm 1
\end{array}\right]=\frac{[K+1 \pm u][1]}{[u+1][K+1]} \\
& W^{V_{1} V_{2}}(z)\left[\begin{array}{lll} 
& K & \\
K \pm 1 & K & \\
& K & K \mp 1]
\end{array}\right]=\frac{[u][K+1 \pm 1]}{[u+1][K+1]}
\end{aligned}
$$

where $q=\exp (-\pi i \tau), z=\exp (2 \pi i \tau u),[x]=\Theta\left(e^{2 \pi i \tau x}\right)$ and $\Theta$ is the Jacobi elliptic theta-function (6.29). This is exactly the famous Baxter solution of the star-triangle relation for the solid-on-solid model [B1].

In the concluding Sect. 8 we discuss some further problems and new directions of research, arising from our results and their comparison with the known facts from conformal field theory and quantum groups.

\section{Affine Lie Algebras and the Knizhnik-Zamolodchikov Equation}

We recall first several standard facts about finite-dimensional simple Lie algebras and fix the notation. Let $\mathfrak{g}$ be a simple Lie algebra over $\mathbf{C}$. We will denote by (,) a symmetric invariant bilinear form on $\mathfrak{g}$ and identify $\mathfrak{g}$ with its dual by means of this form. The form $($,$) is unique up to a constant which we will fix by the requirement$ that $(\theta, \theta)=2$ for the maximal root $\theta$ of $\mathfrak{g}$. We choose a triangular decomposition $\mathfrak{g}=\mathfrak{n}_{+} \oplus \mathfrak{h} \oplus \mathfrak{n}_{-}$, where $\mathfrak{h}$ is a Cartan subalgebra and $\mathfrak{n}_{+}, \mathfrak{n}_{-}$are nilpotent subalgebras 
of $\mathfrak{g}$, corresponding to positive and negative roots $\Delta_{+}$and $\left(-\Delta_{+}\right)$, respectively. We will denote by $\varrho$ the half sum of positive roots, and by $g$ the dual Coxeter number. Let $\left\{x_{i}\right\}_{i=1}^{\operatorname{dim} g}$ be an orthonormal basis of $\mathfrak{g}$, then $C=\sum_{i=1}^{\operatorname{dim} \mathfrak{g}} x_{i}^{2}$ is the Casimir element in the universal enveloping algebra $U(\mathfrak{g})$. Let $\left\{V_{\lambda}\right\}_{\lambda \in P_{++}}$be a set of all irreducible representations indexed by their highest weight, which belongs to the positive cone $P_{++}$of the weight lattice $P$ and we denote $\pi_{\lambda}: \mathfrak{g} \rightarrow$ End $V_{\lambda}$ the action of $\mathfrak{g}$. We will often use the module notation $x v$, instead of $\pi_{\lambda}(x) v, x \in \mathfrak{g}, v \in V_{\lambda}$. We denote by $C(\lambda)$ the value of the Casimir operator in the representation $V_{\lambda}$.

Next we recall some facts about the affine Lie algebra $\hat{\mathfrak{g}}$ associated to $\mathfrak{g}$ (for more details see [Koh, FLM]). By definition $\hat{\mathfrak{g}}=\bigoplus_{n \in \mathbf{Z}} \mathfrak{g}^{n} \oplus \mathbf{C} c$, where $\mathfrak{g}^{n} \cong \mathfrak{g}, n \in \mathbf{Z}$ as vector spaces, and $c$ is in the center of $\hat{\mathfrak{g}}$. Then the commutation relations of the elements $J_{x}^{n} \in \mathfrak{g}^{n}$, corresponding to $x \in \mathfrak{g}$, are

$$
\left[J_{x}^{m}, J_{y}^{n}\right]=J_{[x, y]}^{m+n}+m \delta_{m+n, 0}(x, y) c .
$$

We will identify $\mathfrak{g}$ with the subalgebra $\mathfrak{g}^{0}$ of $\hat{\mathfrak{g}}$ and we will also write $J_{x}$ instead of $x$ for $x \in \mathfrak{g}$. It is often convenient to use the language of the generating functions, namely

$$
\begin{gathered}
J_{x}(z)=J_{x}^{+}(z)-J_{x}^{-}(z), \\
J_{x}^{+}(z)=\sum_{n>0} J_{x}^{-n} z_{n}^{n-1}, \quad J_{x}^{-}(z)=-\sum_{n \geq 0} J_{x}^{n} z^{-n-1},
\end{gathered}
$$

where $z$ is a formal variable. The commutation relations (2.1) admit the following form:

$$
\begin{gathered}
{\left[J_{x}^{ \pm}(z), J_{y}^{ \pm}(w)\right]=\frac{1}{z-w}\left(J_{[x, y]}^{ \pm}(z)-J_{[x, y]}^{ \pm}(w)\right)} \\
{\left[J_{x}^{+}(z), J_{y}^{-}(w)\right]=\frac{1}{z-w}\left(J_{[x, y]}^{+}(z)-J_{[x, y]}^{-}(w)\right)+\frac{c(x, y)}{(z-w)^{2}}}
\end{gathered}
$$

Here $(z-w)^{-1}$ and $(z-w)^{-2}$ should be understood as power series expansions $\frac{1}{z} \sum_{n \geq 0}\left(\frac{w}{z}\right)^{n}$ and $\frac{1}{z^{2}} \sum_{n \geq 1} n\left(\frac{w}{z}\right)^{n-1}$, respectively, and the identities (2.4) and (2.5)

as the identities over $\mathbf{C}\left[z^{ \pm 1}, w^{ \pm 1}\right]$ and $\mathbf{C}\left[z, w^{-1}\right]$, respectively. For more details on the formal calculus of generating functions for affine Lie algebras see [FLM].

Let $\hat{\mathfrak{g}}_{+}=\bigoplus_{n \geq 0} \mathfrak{g}^{n} \oplus \mathbf{C} c$ be the maximal parabolic subalgebra of $\mathfrak{g}$ and $V$ be a $\mathfrak{g}$-module. We introduce in $V$ the structure of the $\hat{\mathfrak{g}}_{+}$-module such that $\mathfrak{g}^{n} V=0$, $n>0, c V=k V$. With each such a module we associate the induced representation of $\hat{\mathfrak{g}}$

$$
V_{k}=\operatorname{Ind}_{\hat{\mathfrak{g}}_{+}}^{\hat{\mathrm{g}}} V .
$$

The space $V_{k}$ has a natural grading consistent with the grading of $\hat{g}$,

$$
V_{k}=\bigoplus_{n \geq 0} V_{k}[-n]
$$


If $V$ is finite dimensional the spaces $V_{k}[n]$ are finite dimensional as well. We define a graded dual module $\left(V_{k}\right)^{*}$ as a linear space

$$
\left(V_{k}\right)^{*}=\bigoplus_{n \geq 0}\left(V_{k}[-n]\right)^{*}
$$

with the following structure of $\hat{\mathfrak{g}}$-module on it:

$$
\left\langle x v^{\prime}, v\right\rangle=-\left\langle v^{\prime}, x v\right\rangle \text {. }
$$

Here $v^{\prime} \in\left(V_{k}\right)^{*}, v \in V_{k}, x \in \hat{\mathfrak{g}}$ and $\langle$,$\rangle is a pairing \left(V_{k}\right)^{*} \otimes V_{k} \rightarrow \mathbf{C}$. The top subspace $V_{k}[0]$ can be naturally identified with $V$. The representation $V_{\lambda, k}$ induced from an irreducible finite-dimensional representation $V_{\lambda}, \lambda \in P_{++}$, of $\mathfrak{g}$ is irreducible for $k \ni \mathbf{Q}$. If $k \in \mathbf{Z}_{+}, V_{\lambda, k}$ is reducible and its factor by the maximal ideal is an integrable dominant highest weight representation for $\lambda \in P_{++}^{k}=\left\{\mu \in P_{++} \mid(\mu, \theta) \leq k\right\}$. Any induced representation $V_{k}$ of $\hat{\mathfrak{g}}$ can be extended to the semidirect product of $\hat{\mathfrak{g}}$ with the Virasoro algebra. In particular, $L_{0}$ is the degree operator on $V_{k}$ and its value on $V_{k}[0]$ called conformal weight, is equal to $h(\lambda)=C(\lambda) / 2(k+g)$ for $V=V_{\lambda}$. We note, however, that in this work the Virasoro algebra is never used and the choice of the shift $h(\lambda)$ in the definition of the degree operator will be motivated also by a certain differential equation.

To any representation $V$ of $\mathfrak{g}$ and a formal variable $z$, we can also associate a representation $V(z)$ of $\hat{\mathfrak{g}}$, in which $k$ is 0 . As a vector space $V(z) \cong V \otimes \mathbf{C}((z))$, where $\mathbf{C}((z))$ denotes the Laurent series in $z$, and $J_{x}^{n}$ acts by $x \otimes z^{n}$. One of the central objects of the conformal field theory associated to representations of affine Lie algebras are the intertwining operators

$$
\Phi(z): V_{\mu, k} \rightarrow V_{\nu, k} \otimes V_{\lambda}(z) z^{-h(\lambda)-h(\mu)+h(\nu)},
$$

where $\lambda, \mu, \nu \in P_{++}$, and the shift in grading $z^{-h(\lambda)-h(\mu)+h(\nu)}$ comes from the grading of representations and will play an important role. A fixed choice of an element $v \in V_{\lambda}^{*}$ gives rise to an operator

$$
\Phi_{v}(z): V_{\mu, k} \rightarrow V_{\nu, k} \otimes \mathbf{C}((z)) z^{-h(\lambda)-h(\mu)+h(\nu)},
$$

or in the component form

$$
\Phi_{v}(z)=\sum_{n \in \mathbf{Z}} \Phi_{v}[n] z^{-n-h(\lambda)-h(\mu)+h(\nu)} .
$$

Since the intertwining property of $\Phi(z)$ does not depend on a multiplication by any power series of $z$ we require in addition that the grading (2.10) is consistent with the graded structures of the representations,

$$
\Phi_{v}[n]: V_{\nu, k}[m] \rightarrow V_{\mu, k}[m+n] .
$$

By definition the intertwining operator $\Phi_{v}(z)$ satisfies

$$
\left[J_{x}^{ \pm}(w), \Phi_{v}(z)\right]=\frac{1}{z-w} \Phi_{x v}(z),
$$

where $(z-w)^{-1}$ should be underestood as the corresponding positive or negative power series in $\left(\frac{w}{z}\right)$ depending on the sign of $J_{x}^{ \pm}(w)$. We also define a normal
ordering

$$
: J_{x}(z) \Phi_{v}(z):=J_{x}^{+}(z) \Phi_{v}(z)-\Phi_{v}(z) J_{x}^{-}(z) .
$$


Remark 2.1. Heuristically, when $z$ is set to be a complex variable, the definition (2.13) is motivated by the following expression often used in physics literature [BPZ]:

$$
: J_{x}(z) \Phi_{v}(z):=\frac{1}{2 \pi i} \int_{C_{z}} T\left(J_{x}(\zeta) \Phi_{v}(z)\right) \frac{d \zeta}{\zeta-z}
$$

where $C_{z}$ is a circle with the center in $z$ with the counterclockwise orientation and $T$ is the radial ordering of operators, namely $T\left(J_{x}(\zeta) \Phi_{v}(z)\right)=J_{x}(\zeta) \Phi_{v}(z)$ if $|\zeta|>|z|$ and $\Phi_{v}(z) J_{x}(\zeta)$ if $|z|>|\zeta|$. The latter can be rewritten, using the Cauchy theorem for an analytic function of $\zeta$ with the three singular points $0, z$ and $\infty$, in the form

$$
: J_{x}(z) \Phi_{v}(z):=\frac{1}{z \pi i} \int_{C_{R}} J_{x}(\zeta) \Phi_{v}(z) \frac{d \zeta}{\zeta-z}-\frac{1}{2 \pi i} \int_{C_{r}} \Phi_{v}(z) J_{x}(\zeta) \frac{d \zeta}{\zeta-z}
$$

where $C_{R}$ and $C_{r}$ are the circles with center in 0 and the counterclockwise orientation of radii $R$ and $r$, respectively, and $R>|z|>r$. Here $z$ and $\zeta$ are complex variables and all such identities should be understood in the weak sense, i.e. as the equality of arbitrary matrix coefficients of the corresponding operators. For more detail on relations between the formal variable identities and their complex analytic counterparts, see the Appendix in [FLM]. One can show that in this form (2.14) the right-hand side is well defined and then this definition of normal ordering is immediately reduced to (2.13).

We deduce first an operator analogue of the linear differential equation for $\Phi_{v}(z)$, which we consider one of the cornerstones of the conformal field theory associated to the highest weight representations of the affine Lie algebra $\hat{\mathfrak{g}}$ (cf. [KZ]).

Theorem 2.1. The intertwining operator $\Phi_{v}(z)$ defined as (2.8)-(2.12) satisfies the following differential equation:

$$
(k+g) \frac{d}{d z} \Phi_{v}(z)=\sum_{a}: J_{a}(z) \Phi_{a v}(z):
$$

where the sum is taken over an orthonormal basis of $\mathfrak{g}$.

Proof. We will give an induction proof. Let us first consider the matrix coefficient of $\Phi_{v}(z)$ on the top level. One has

$$
\left\langle v_{\infty}, \Phi_{v}(z) v_{0}\right\rangle=\frac{I\left(v_{\infty} \otimes v \otimes v_{0}\right)}{z^{h(\lambda)+h(\mu)-h(\nu)}}
$$

where $v \in V_{\lambda}^{*}, v_{0} \in V_{\mu}^{-k}[0] \cong V_{\mu}, v_{\infty} \in\left(V_{\nu}^{k}[0]\right)^{*} \cong\left(V_{\nu}\right)^{*}$, and $I$ is a g-invariant functional on $V_{\nu}^{*} \otimes V_{\lambda}^{*} \otimes V_{\mu}$. Then we find

$$
\begin{aligned}
\left\langle v_{\infty}, \frac{d}{d z} \Phi_{v}(z) v_{0}\right\rangle=-(h(\lambda)+ & h(\mu)-h(\nu)) \frac{I\left(v_{\infty} \otimes v \otimes v_{0}\right)}{z^{h(\lambda)+h(\mu)-h(\nu)+1}} \\
\left\langle v_{\infty}, \sum_{a}: J_{a}(z) \Phi_{a v}(z): v_{0}\right\rangle & =z^{-1} \sum_{a}\left\langle v_{\infty}, \Phi_{a v}(z) J_{a} v_{0}\right\rangle \\
& =\sum_{a} \frac{I\left(v_{\infty} \otimes J_{a} v \otimes J_{a} v_{0}\right)}{z^{h(\lambda)+h(\mu)-h(\nu)+1}}
\end{aligned}
$$


Using the $\mathfrak{g}$-invariance of $I$ we get

$$
\begin{aligned}
\sum_{a} I\left(v_{\infty} \otimes J_{a} v \otimes J_{a} v_{0}\right) & =\frac{1}{2} I\left(\operatorname{Id} \otimes(\Delta(C)-C \otimes 1-1 \otimes C) v_{\infty} \otimes v \otimes v_{0}\right) \\
& =\frac{1}{2}\left(C(\nu)-C(\mu)-C(\lambda) I\left(v_{\infty} \otimes v \otimes v_{0}\right)\right.
\end{aligned}
$$

where $\Delta$ is a standard comultiplication. Now, using $h(\lambda)=C(\lambda) / 2(k+g)$ we obtain (2.15) at the top level.

The induction step is an immediate corollary of the following identities:

$$
\begin{gathered}
{\left[J_{a}^{ \pm}(w)-\frac{1}{z-w} J_{a},(k+g) \frac{d}{d z} \Phi_{v}(z)-\sum_{a}: J_{b}(z) \Phi_{b v}(z):\right]=0,} \\
{\left[J_{a}, \Phi_{v}(z)\right]=\Phi_{a v}(z) .}
\end{gathered}
$$

In fact, since $V_{\lambda, k}$ is generated by its top level we only need to prove that the commutator of $J_{a}^{n}, n \in \mathbf{Z}$, with the left- and right-hand sides of (2.15) has the same matrix coefficients. Then by induction arbitrary matrix coefficients are reduced to the top level, where Eq. (2.15) has been checked.

The proof of the identities (2.19) uses only the commutation relations (2.4), (2.5) and (2.12). One has

$$
\begin{gathered}
{\left[J_{a}^{+}(w)-\frac{1}{z-w} J_{a}, \frac{d}{d z} \Phi_{v}(z)\right]=-\frac{1}{(w-z)^{2}} \Phi_{a v}(z)} \\
{\left[J_{a}^{+}(w)-\frac{1}{z-w} J_{a}, \Phi_{b v}(z) J_{b}^{+}(z)-J_{b}^{-}(z) \Phi_{b v}(z)\right]} \\
=\frac{1}{z-w} \Phi_{b v}(z) J_{[a b]}^{+}(w)-\frac{1}{z-w} J_{[a, b]}^{+}(w) \Phi_{b v}(z)-\frac{k}{(z-w)^{2}} \Phi_{a v}(z) \\
=-\frac{1}{(z-w)^{2}} \Phi_{[a b] b v}(z)-\frac{k}{(z-w)^{2}} \Phi_{a v}(z)=-\frac{k+g}{(z-w)^{2}} \Phi_{a v}(z)
\end{gathered}
$$

since

$$
\sum_{a}[a b] b v=\frac{1}{2} \sum_{b}[b[b a]] v=\frac{1}{2} C(\theta) a v=g a v,
$$

and similarly for $J_{a}^{-}(w)$.

One can also reformulate the statement of the theorem in terms of the intertwining operators $\Phi(z)$. Let us introduce the generating function $J(z)=\sum_{a} J_{a} \otimes J_{a}$ acting in the tensor product $V_{\nu, k} \otimes V_{\lambda}(z)$. Then the operator differential equation (2.15) admits the especially elegant form

$$
(k+g) \frac{d}{d z} \Phi(z)=: J(z) \Phi(z):
$$

where $: J(z) \Phi(z):=\sum_{a}\left(J_{a}^{+}(z) \otimes J_{a}\right) \Phi(z)-\sum_{a}\left(1 \otimes J_{a}\right) \Phi(z) J_{a}^{-}(z)$. The equation in this form will have a natural generalization for quantum affine algebras.

The proof of the theorem implies the existence of the intertwining operators satisfying (2.16) on the top level. This shows that the dimension of the linear space of intertwining operators (2.8) is equal to $\operatorname{dim} \operatorname{Homg}\left(V_{\mu}, V_{\nu} \otimes V_{\lambda}\right)$.

The differential equation for the intertwining operator now immediately implies the Knizhnik-Zamolodchikov equation [KZ]. 
For abbreviation we will write

$$
\Phi_{1}\left(z_{1}\right) \ldots \Phi_{N-1}\left(z_{N-1}\right) \Phi_{N}\left(z_{N}\right) \equiv\left(\Phi_{1}\left(z_{1}\right) \otimes 1 \cdots \otimes 1\right) \ldots\left(\Phi_{N-1}\left(z_{N-1}\right) \otimes 1\right) \Phi_{N}\left(z_{N}\right) .
$$

Proposition 2.2. Matrix coefficients of a product of intertwining operators

$$
\Psi=\left\langle\cdot, \Phi_{1}\left(z_{1}\right) \ldots \Phi_{N}\left(z_{N}\right) \cdot\right\rangle \in V^{(0)} \otimes V_{\lambda_{1}} \otimes \cdots \otimes V_{\lambda_{N}} \otimes\left(V^{(N+1)}\right)^{*},
$$

where $V_{k}^{(0)}$ is the target space of $\Phi_{1}\left(z_{1}\right)$ and $V_{k}^{(N+1)}$ is the source space of $\Phi_{N}\left(z_{N}\right)$, satisfy the following system of partial differential equations

$$
(k+g) \frac{\partial \Psi}{\partial z_{i}}=\sum_{j \pm i} \frac{\Omega_{i j}}{z_{i}-z_{j}} \Psi
$$

where $z_{N+1}=0$ and $\Omega_{i j}=\sum_{a} 1 \otimes \cdots \otimes a \otimes \cdots \otimes a \otimes \cdots \otimes 1$, a appears at the $i^{\text {th }}$ and $j^{\text {th }}$ position.

Proof. In order to avoid the vector notation, one can introduce a scalar function

$$
\psi=\left\langle\Psi, v_{0} \otimes v_{1} \otimes \cdots \otimes v_{N-1} \otimes v_{N}\right\rangle .
$$

Then, applying (2.15) and (2.12) one obtains

$$
\begin{aligned}
\frac{\partial \psi}{\partial z_{i}}= & \sum_{a}\left\langle v_{0}, \ldots\left(J_{a}^{+}\left(z_{i}\right) \Phi_{a v_{i}}\left(z_{i}\right)-\Phi_{a v_{i}}\left(z_{i}\right) J_{a}\left(z_{i}\right)\right) \ldots v_{N+1}\right\rangle \\
= & \sum_{a} \sum_{j<i} \frac{1}{z_{i}-z_{j}}\left\langle v_{0} \ldots \Phi_{a v_{j}}\left(z_{j}\right) \ldots \Phi_{a v_{i}}\left(z_{i}\right) \ldots v_{N+1}\right\rangle \\
& +\sum_{a} \sum_{j<i} \frac{-1}{z_{j}-z_{i}}\left\langle v_{0}, \ldots \Phi_{a v_{i}}\left(z_{i}\right) \ldots \Phi_{a v_{j}}\left(z_{j}\right) \ldots v_{N+1}\right\rangle
\end{aligned}
$$

This is equivalent to (2.21) for the vector-valued function $\Psi$. We note that in the quantum case we will have to work exclusively in the "vector" notation.

Finally, one can deduce from the derivation of the Knizhnik-Zamolodchikov equation and also easily check directly

Proposition 2.3. The system (2.21) is consistent, i.e

$$
\left[(k+g) \frac{\partial}{\partial z_{i}}-\sum_{j \pm i} \frac{\Omega_{i j}}{z_{i}-z_{j}},(k+g) \frac{\partial}{\partial z_{k}}-\sum_{l \neq k} \frac{\Omega_{k l}}{z_{k}-z_{l}}\right]=0 .
$$

The consistency property of the Knizhnik-Zamolodchikov equation directly follows from the fact that

$$
r_{i j}\left(z_{i}-z_{j}\right)=\frac{\Omega_{i j}}{z_{i}-z_{j}}
$$

is a solution of the classical Yang-Baxter equation

$$
\begin{aligned}
{\left[r_{12}\left(z_{1}-z_{2}\right), r_{13}\left(z_{1}-z_{3}\right)\right]+} & {\left[r_{12}\left(z_{1}-z_{2}\right), r_{23}\left(z_{2}-z_{3}\right)\right] } \\
+ & {\left[r_{13}\left(z_{1}-z_{3}\right), r_{23}\left(z_{2}, z_{3}\right)\right]=0 . }
\end{aligned}
$$

Besides the rational solution such as (2.22) the classical Yang-Baxter equation also possesses trigonometric and elliptic type of solutions studied in [KS, BD]. An example 
of a trigonometric solution for any simple Lie algebra $\mathfrak{g}$ (written in a multiplicative parametrization) is well known

$$
\tilde{r}_{\imath j}\left(z_{i} / z_{j}\right)=\frac{\tilde{r}_{i j} z_{i}+\tilde{r}_{j i} z_{j}}{z_{i}-z_{j}}
$$

where

$$
\tilde{r}=\frac{1}{2} \sum_{i=1}^{r} h_{i} \otimes h_{i}+\sum_{\alpha \in \Delta_{+}} x_{\alpha} \otimes x_{-\alpha}
$$

is a "half-Casimir," $\left\{h_{i}\right\}_{i=1}^{r}$ is a basis of $\mathfrak{h}$ and $x_{\alpha} \in \mathfrak{g}_{\alpha}, \alpha \in \Delta$, and $\left\langle x_{\alpha}, x_{-\alpha}\right\rangle=1$ for all $\alpha \in \Delta$.

It turns out that a simple transformation of the Knizhnik-Zamolodchikov equation (2.21) allows us to rewrite it in the trigonometric form with $r_{i j}\left(z_{i}-z_{j}\right)$ replaced by $\tilde{r}_{i j}\left(z_{i} / z_{j}\right)$. This transformation is related however to a more fundamental fact of changing the polarization of the affine Lie algebra $\hat{\mathfrak{g}}$. Instead of "parabolic type" polarization (2.2), (2.3) we can choose the "Borel type" polarization

$$
\tilde{J}_{x}(z)=\tilde{J}_{x}^{+}(z)-\tilde{J}_{x}^{-}(z)
$$

where $\tilde{J}_{x}(z)=z J_{x}(z)$,

$$
\tilde{J}_{x}^{ \pm}(z)= \pm\left(J_{\frac{1}{2} x^{0}+x^{\mp}}^{0}+\sum_{n>0} J_{x}^{\mp n} z^{ \pm n}\right)
$$

and $x^{+}, x^{0}, x^{-}$are, respectively, components of $x \in \mathfrak{g}$ in the triangular decomposition $\mathfrak{g}=\mathfrak{n}_{+} \oplus \mathfrak{h} \oplus \mathfrak{n}_{-}$. We can then define a normal ordering corresponding to the new polarization

$$
\vdots \tilde{J}_{x}(z) \Phi_{v}(z) \vdots=\tilde{J}_{x}^{+}(z) \Phi_{v}(z)-\Phi_{v}(z) \tilde{J}_{x}^{-}(z)
$$

and similarly $\vdots \tilde{J}(z) \Phi(z) \vdots$. One immediately obtains

Proposition 2.4. Normal orderings are related as follows:

$$
z: J(z) \Phi(z):=\vdots \tilde{J}(z) \Phi(z) \vdots-1 \otimes\left(-\frac{C}{2}+\varrho\right) \Phi(z),
$$

where $C$ is the Casimir operator and $\varrho$ is the half sum of positive roots.

It is also natural to introduce

$$
\tilde{\Phi}(z)=z^{-\frac{1 \otimes C}{2(k+g)}} \Phi(z) .
$$

Then the differential equation (2.20) admits the form

$$
(k+g) z \frac{d}{d z} \tilde{\Phi}(z)=\vdots \tilde{J}(z) \tilde{\Phi}(z) \vdots-1 \otimes \varrho \tilde{\Phi}(z) .
$$

Now, choosing $v_{0}$ and $v_{N+1}$ to be the highest weight vectors we obtain the trigonometric form of the Knizhnik-Zamolodchikov equation for $\Psi$ from

\section{Corollary 2.1.}

$$
(k+g) z_{i} \frac{\partial \tilde{\Psi}}{\partial z_{i}}=\left\{\sum_{j \neq i} \tilde{r}_{i j}\left(z_{i} / z_{j}\right)+\frac{1}{2} \pi_{i}\left(\left(\lambda_{0}+\varrho\right)+\left(\lambda_{n+1}+\varrho\right)\right)\right\} \tilde{\Psi},
$$


where

$$
\tilde{\Psi}=\left\langle v_{0}, \tilde{\Phi}_{1}\left(z_{1}\right) \ldots \tilde{\Phi}_{N}\left(z_{N}\right) v_{N}\right\rangle .
$$

This equation çan also be obtained directly from the Knizhnik-Zamolodchikov equation (2.21) using the fact that

$$
\sum_{j=1}^{n+1} \tilde{r}_{j i}=\tilde{r}_{0 i}, \quad \text { and } \quad \tilde{r}_{i i}=\pi_{i}\left(\frac{1}{2} C-\varrho\right) .
$$

Remark 2.2. We have seen that the parabolic and Borel type polarizations of the affine Lie algebra $\hat{\mathfrak{g}}$ are related by a simple transformation, which gives rise to the corresponding relation between the rational and trigonometric form of the KnizhnikZamolodchikov equation. However these two polarizations lead to two different Lie bialgebra structures on $\hat{\mathfrak{g}}$. Furthermore the quantization of two bialgebras yields two different quantum analogues of $\hat{\mathfrak{g}}$, namely "full" Yangian $\hat{Y}(\mathfrak{g})$ (or the quantum double of the Yangian $Y(\mathfrak{g})[\mathrm{S}])$ in the rational case and quantum affine algebra $U_{q}(\hat{\mathfrak{g}})$ in the trigonometric case. A generalization of the results of this section to the above quantum analogues is our main goal.

Remark 2.3. We also would like to note that one can deduce a differential equation for

$$
\Psi=\left.\operatorname{tr}\right|_{V_{\lambda, k}}\left(\tilde{\Phi}_{1}\left(z_{1}\right) \ldots \tilde{\Phi}_{N}\left(z_{N}\right) q^{L_{0}}\right)
$$

where $L_{0} \cdot V_{\lambda, k}[n]=(h(\lambda)-n) V_{\lambda, k}[n]$. Then $\tilde{r}(z)$ is likely to be replaced by an elliptic solution of the classical Yang-Baxter equation on $\mathfrak{g}$-invariant subspace in $V_{1} \otimes \cdots \otimes V_{N}$

We would like to recall briefly the approach of [TK] in a slightly modified form, which we will extend to the quantum case in Sect. 6. We have seen that the solutions of the Knizhnik-Zamolodchikov equation can be obtained as matrix coefficients of the product of intertwining operators

$$
\Phi_{\jmath}\left(z_{\jmath}\right): V_{\lambda_{\jmath, k}} \rightarrow V_{\lambda_{\jmath-1, k}} \otimes V_{\mu_{j}}\left(z_{j}\right) z^{h_{\left(\lambda_{\jmath-1}\right)}-h_{\left(\lambda_{j}\right)}-h_{\left(\mu_{\jmath}\right)}} .
$$

In this case we will need to specify the source and the target space of the intertwining operator (2.32). We will use the notation $\Phi_{j}\left(z_{j}\right)_{\lambda_{j}}^{\lambda_{j-1}}$. By the proof of Theorem 2.1, these operators are in one-to-one correspondence with elements of the vector space

$$
H_{\lambda_{\jmath}}^{\lambda_{j-1} \mu_{j}}=\operatorname{Hom}_{\mathfrak{g}}\left(V_{\lambda_{\jmath}}, V_{\lambda_{j-1}} \otimes V_{\mu_{\jmath}}\right) .
$$

We will write $\Phi_{j}\left(z_{j} \mid a\right), a \in H_{\lambda_{j}}^{\lambda_{\jmath-1} \mu_{j}}$, to specify a particular intertwining operator. Then $\Phi_{\jmath}(z \mid \cdot)$ maps $H_{\lambda_{j}}^{\lambda_{\jmath-1} \mu_{j}}$ into the space of intertwiners of a given type. Thus any element of the space

$$
H_{\lambda_{1}}^{\lambda_{0} \mu_{1}} \otimes \cdots \otimes H_{\lambda_{j}}^{\lambda_{\jmath-1} \mu_{\jmath}} \otimes H_{\lambda_{\jmath+1}}^{\lambda_{j} \mu_{j+1}} \otimes \cdots \otimes H_{\lambda_{N}}^{\lambda_{N-1} \mu_{N}}
$$

gives a $\mathfrak{g}$-invariant solution of the Knizhnik-Zamolodchikov equation (2.21). We obtain these solutions in terms of the formal power series in $\frac{z_{2}}{z_{1}}, \ldots, \frac{z_{N}}{z_{N-1}}$ and the general theory of linear differential equations asserts that these series are analytic functions in the domain $\left|z_{1}\right| \gg\left|z_{2}\right| \gg \cdots \gg\left|z_{N}\right|$. The analytic continuation of 
the solutions into the domain, where $\left|z_{i+1}\right| \gg\left|z_{i}\right|$, and the rest of the order is unchanged can be done in two different ways $\left(\arg \frac{z_{i}(t)}{z_{i+1}(t)}>0\right.$ or $<0$ at the point $\left.\left|z_{i}(t)\right|=\left|z_{i+1}(t)\right|, t \in[0,1]\right)$ and defines a map of $(2.34)$ into the space

$$
\bigoplus_{\lambda_{j}^{\prime}} H_{\lambda_{1}}^{\lambda_{0} \mu_{1}} \otimes \cdots \otimes H_{\lambda_{j}^{\prime}}^{\lambda_{j-1} \mu_{j+1}} \otimes H_{\lambda_{j+1}}^{\lambda_{j}^{\prime} \mu_{j}} \otimes \cdots \otimes H_{\lambda_{N}}^{\lambda_{N-1} \mu_{N}}
$$

One can show from the analysis of the Knizhnik-Zamolodchikov equations (2.21) [see Sect. 6 for the case of $\left.U_{q}(\hat{\mathfrak{g}})\right]$ that this map has a local form

$$
1 \otimes \cdots \otimes B_{\mu_{j} \mu_{j+1}}^{ \pm}\left[\begin{array}{ccc} 
& \lambda_{j-1} & \\
\lambda_{j}^{\prime} & & \lambda_{j}
\end{array}\right] \otimes \cdots \otimes 1
$$

where

$$
\begin{aligned}
& B_{\mu_{j} \mu_{j+1}}^{ \pm}\left[\begin{array}{ccc}
\lambda_{j}^{\prime} & \lambda_{j-1} & \\
& \lambda_{j+1} & \lambda_{j}
\end{array}\right]: H_{\lambda_{j}}^{\lambda_{j-1} \mu_{j}} \otimes H_{\lambda_{j+1}}^{\lambda_{j} \mu_{j+1}} \\
& \quad \rightarrow\left(H_{\lambda_{j}^{\prime}}^{\lambda_{j-1} \mu_{j+1}} \otimes H_{\lambda_{j}^{\prime}}^{\lambda_{j}^{\prime} \mu_{j+1}}\right)
\end{aligned}
$$

will be called the braiding map. All the structure about monodromies of the KnizhnikZamolodchikov equation is encoded in this map.

Remark 2.4. Since the braiding map (2.39) arises from $N=2$ case of KnizhnikZamolodchikov equation, the whole structure of monodromies is reduced to this case.

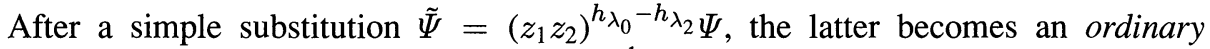
differential equation in a variable $z=z_{1} z_{2}^{-1}$ with three singular points $z=0,1$ and $\infty$. In the simplest nontrivial case, when $\hat{\mathfrak{g}}=\widehat{\mathfrak{s l}(2)}$ and $m=1$ in $\lambda_{2}=\mu_{1}+\mu_{2}+\lambda-m \alpha$ the system of differential equations has order 2 and the solutions are given in terms of the Gauss hypergeometric function $F(a, b, c ; z)$. General $m$ and $N$ give various generalizations of the hypergeometric function studied in [FZa, DFa, SV1, SV2]. We will consider a different generalization of the Gauss function arising in connection to the quantum analogues of $\widehat{\mathfrak{s l}(2)}$ in Sect. 7.

Since the solution of the Knizhnik-Zamolodchikov equation can be analytically continued from formal power series to complex values of $z_{i}$ we can define the analytic continuation of the product of intertwining operators $\mathscr{Q}^{ \pm}\left(\Phi_{1}\left(z_{1}\right) \Phi_{2}\left(z_{2}\right)\right)$, where \pm corresponds to two nonhomotopic paths as above.

Theorem 2.2. Intertwining operators satisfy the following exchange algebra:

$$
\begin{aligned}
P_{12} & \left.\mathscr{\odot}^{ \pm}\left(\Phi_{1}\left(z_{1}\right) \mid \cdot\right)_{\lambda_{1}}^{\lambda_{0}} \Phi_{2}\left(z_{2} \mid \cdot\right)_{\lambda_{2}}^{\lambda_{1}}\right) \\
& =\sum_{\lambda_{1}^{\prime}} \mathscr{\odot}^{\mp}\left(\Phi_{2}\left(z_{2} \mid \cdot\right)_{\lambda_{1}^{\prime}}^{\lambda_{0}} \Phi_{1}\left(z_{1} \mid \cdot\right)_{\lambda_{2}}^{\lambda_{1}^{\prime}}\right) B_{\mu_{1} \mu_{2}}^{ \pm}\left[\begin{array}{ccc}
\lambda_{1}^{\prime} & & \lambda_{1}
\end{array}\right],
\end{aligned}
$$

where $P_{12}$ is the permutation of the factors in the tensor product $V_{\mu_{1}} \otimes V_{\mu_{2}}$ 


\section{Corollary 2.2.}

$$
\begin{aligned}
& \sum_{\lambda_{1}^{\prime}} B_{\mu_{1} \mu_{2}}^{ \pm}\left[\begin{array}{ccc}
\lambda_{1}^{\prime \prime} & \lambda_{0} & \lambda_{1}^{\prime} \\
& \lambda_{2} &
\end{array}\right] B_{\mu_{2} \mu_{1}}^{\mp}\left[\begin{array}{ccc}
\lambda_{1}^{\prime} & \lambda_{0} & \\
& \lambda_{2} & \lambda_{1}
\end{array}\right]=\delta_{\lambda_{1}^{\prime \prime} \lambda_{1}} \\
& \sum_{\bar{\lambda}_{2}}\left(1 \otimes B_{\mu_{1} \mu_{2}}^{ \pm}\left[\begin{array}{ccc} 
& \lambda_{1}^{\prime} & \\
\lambda_{2}^{\prime} & & \lambda_{1}^{\prime} \\
& \lambda_{3} &
\end{array}\right]\right)\left(B_{\mu_{1} \mu_{3}}^{ \pm}\left[\begin{array}{ccc} 
& \lambda_{0} & \\
\lambda_{1}^{\prime} & \bar{\lambda}_{2} & \lambda_{1}^{\prime}
\end{array}\right] \otimes 1\right) \\
& \times\left(1 \otimes B_{\mu_{2} \mu_{3}}^{ \pm}\left[\begin{array}{lll} 
& \lambda_{1} & \\
\bar{\lambda}_{2} & & \lambda_{2} \\
& \lambda_{3} &
\end{array}\right]\right) \\
& =\sum_{\bar{\lambda}_{1}}\left(B_{\mu_{2} \mu_{3}}^{ \pm}\left[\begin{array}{ccc} 
& \lambda_{0} & \\
\lambda_{1}^{\prime} & & \bar{\lambda}_{1} \\
& \lambda_{2}^{\prime} &
\end{array}\right] \otimes 1\right)\left(1 \otimes B_{\mu_{1} \mu_{3}}^{ \pm}\left[\begin{array}{ccc}
\lambda_{1}^{\prime} & \bar{\lambda}_{1} & \\
& \lambda_{3} & \lambda_{2}
\end{array}\right]\right) \\
& \times\left(B_{\mu_{1} \mu_{2}}^{ \pm}\left[\begin{array}{ccc}
\bar{\lambda}_{1} & \lambda_{0} & \\
& \lambda_{2} & \lambda_{1}
\end{array}\right] \otimes 1\right) \text {. }
\end{aligned}
$$

The important special case of the braiding map $B_{\mu_{1} \mu_{2}}^{ \pm}\left[\begin{array}{cccc} & \lambda_{0} & \\ \lambda_{1}^{\prime} & & \lambda_{1} \\ & \lambda_{2} & \end{array}\right]$ arises when $\lambda_{0}=0$. We denote it by $A_{\mu_{1} \mu_{2}}^{ \pm}\left[\lambda_{2}\right]$, since in this case $\lambda_{1}=\mu_{1}$ and $\lambda_{1}^{\prime}=\mu_{2}$. We can also identify $H_{\lambda}^{0 \lambda} \cong \mathbf{C}$ by singling out the intertwiner corresponding to $1 \in \mathbf{C}$,

$$
\left\langle v_{0}, \Phi(z) v\right\rangle=v
$$

where $v_{0} \in V_{0, k}^{*}$ is a fixed lowest weight vector. Using this identification, we obtain

$$
A_{\mu_{1} \mu_{2}}^{ \pm}[\lambda]: H_{\lambda}^{\mu_{1} \mu_{2}} \rightarrow H_{\lambda}^{\mu_{2} \mu_{1}}
$$

The relations for $B^{ \pm}$can be best understood from the point of view of a braided monoidal category, which we briefly describe [ML, RT]. By definition of a monoidal category $\mathscr{C}$, there is a bifunctor (tensor product) $\otimes: \mathscr{C} \times \mathscr{C} \rightarrow \mathscr{C}$, identity object $I$, and three natural isomorphisms

$$
\begin{gathered}
\alpha_{X, Y, Z}:(X \otimes Y) \otimes Z \rightarrow X \otimes(Y \otimes Z), \\
\lambda_{X}: J \otimes X \cong X, \quad \varrho_{X}: X \otimes I \cong X,
\end{gathered}
$$

so that $\alpha$ satisfies the pentagonal diagram [ML], $\alpha, \lambda, \varrho$ satisfy elementary triangular diagrams $\lambda_{X \otimes Y} \circ \alpha_{I, X, Y}=\lambda_{X} \otimes \operatorname{id}_{Y},\left(\operatorname{id}_{X} \otimes \lambda_{Y}\right) \circ \alpha_{X, Y, I}=\varrho_{X \otimes Y}$ and $\lambda_{I}=\varrho_{I}$. A monoidal category is called a braided monoidal category if in addition there are two natural isomorphisms

$$
\gamma_{X, Y}^{ \pm}: X \otimes Y \rightarrow Y \otimes X
$$

so that

$$
\begin{gathered}
\gamma_{X, Y}^{ \pm} \circ \gamma_{Y, X}^{\mp}=\mathrm{id}_{X \otimes Y}, \\
\left(\mathrm{id}_{Y} \otimes \gamma_{X, Z}^{ \pm} \circ \alpha_{Y, X, Z} \circ\left(\gamma_{X, Y}^{ \pm} \otimes \mathrm{id}_{Z}\right)=\alpha_{Y, Z, X} \circ \gamma_{X, Y \otimes Z}^{ \pm} \circ \alpha_{X, Y, Z},\right.
\end{gathered}
$$

and also $\varrho_{X}=\lambda_{X} \circ \gamma_{X, I}^{ \pm}$. In this paper we will call tensor category an abelian braided monoidal category. We call pre-tensor category a tensor category without the axiom given by the pentagonal diagram. 
Let us introduce a natural transformation which we call braiding,

$$
\begin{gathered}
\beta_{X, Y, Z}^{ \pm}:(X \otimes Y) \otimes Z \rightarrow(X \otimes Z) \otimes Y, \\
\beta_{X, Y, Z}^{ \pm}=\gamma_{Y, X \otimes Z}^{ \pm} \circ \alpha_{Y, X, Z} \circ\left(\gamma_{X, Y}^{\mp} \otimes \mathrm{id}_{Z}\right) .
\end{gathered}
$$

One can now reformulate the axioms of the (pre-) tensor category using the braiding constraint. In particular, one easily checks

Proposition 2.6. The axioms (2.47) and (2.48) are equivalent to the following ones:

$$
\begin{gathered}
\beta_{\cdot, X, Y}^{ \pm} \circ \beta_{\cdot, Y, X}^{\mp}=\mathrm{id} \\
\left(\beta_{\cdot, Y, Z}^{ \pm} \otimes \operatorname{id}_{X}\right) \circ \beta_{\cdot \otimes Y, X, Z}^{ \pm} \circ\left(\beta_{\cdot, X, Y}^{ \pm} \otimes \mathrm{id}_{Z}\right) \\
=\beta_{\cdot \otimes Z, X, Y}^{ \pm} \circ\left(\beta_{\cdot, X, Z}^{ \pm} \otimes \operatorname{id}_{Y}\right) \circ \beta_{\cdot \otimes X, Y, Z}^{ \pm}
\end{gathered}
$$

Now let us assume that $\mathscr{C}$ is semisimple, namely that every object is a direct sum of simple objects $X_{i}, i \in \Omega$, and $I \cong \mathbf{C}, \operatorname{Hom}\left(X_{i}, X_{\imath}\right) \cong \mathbf{C}$. Then we have:

$$
X_{i} \otimes X_{j}=H_{k}^{i j} \otimes X_{k}
$$

where $H_{k}^{i j}$ is a vector space. Let

$$
\left(X_{i} \otimes X_{j}\right) \otimes X_{k}=\bigoplus_{m, n} H_{m}^{i j} \otimes H_{n}^{m k} \otimes X_{n} .
$$

Then $\beta_{X, Y, Z}^{ \pm}$induces on the vector space $\bigoplus_{m} H_{m}^{i j} \otimes H_{n}^{m k}$ a linear map

$$
B_{\jmath k}^{ \pm}\left[\begin{array}{ccc} 
& i & \\
m^{\prime} & & m \\
& n &
\end{array}\right]: H_{m}^{\imath j} \otimes H_{n}^{m k} \rightarrow H_{m^{\prime}}^{i k} \otimes H_{n}^{m^{\prime} j}
$$

and the identities (2.51) and (2.52) imply the identities (2.40) and (2.41), respectively.

Thus we have seen that the monodromy of Knizhnik-Zamolodchikov equation yields a semisimple pre-tensor category with simple objects indexed by $P_{++}$. Operator product expansions of intertwining operators or further analysis of the KnizhnikZamolodchikov equation reveals that the pentagon diagram holds and we have in fact a tensor category, which will be denoted by $\operatorname{Mon}_{k}(\hat{\mathfrak{g}})$. It also has an additional structure of rigidity and balancing, which will be important for us in the case of another tensor category associated to the finite dimensional representations of quantum groups considered in the next section.

\section{Quantum Algebra $U_{q}(\mathbf{g})$}

Let $q$ be a formal paramter, $\mathfrak{g}$ be a simple Lie algebra with fixed Cartan matrix $A=\left(a_{i j}\right), i, j=1, \ldots$, rank $\mathfrak{g}$ and $d_{i}=1,2,3$ such that $d_{i} a_{i j}=d_{j} a_{j i}$.

Definition 3.1 [Dr1, J2]. The algebra $U_{q}(\mathfrak{g})$ is an associative algebra over formal power series $\mathbf{C}[[q-1]]$ with generators $e_{i}, f_{\imath}$ and invertible $k_{i}, i=1, \ldots, l=\operatorname{rank} \mathfrak{g}$ 
and with relations:

$$
\begin{gathered}
k_{i} k_{j}=k_{j} k_{i}, \quad k_{i} e_{j}=q_{i}^{a_{i j}} e_{j} k_{i}, \quad k_{i} f_{j}=q_{i}^{-a_{i j}} f_{j} k_{i}, \\
e_{i} f_{j}-f-j e_{i}=\delta_{i j} \frac{k_{i}-k_{i}^{-1}}{q_{i}-q_{i}^{-1}}, \\
\sum_{k=0}^{1-a_{i j}}(-1)^{k}\left[\begin{array}{c}
1-a_{i j} \\
k
\end{array}\right]_{q_{i}} e_{i}^{1-a_{i j}-k} e_{j} e_{i}^{k}=0, \quad i \neq j, \\
\sum_{k=0}^{1-a_{i j}}(-1)^{k}\left[\begin{array}{c}
1-a_{i j} \\
k
\end{array}\right]_{q_{i}} f_{i}^{1-a_{i j}-k} f_{j} f_{i}^{k}=0, \quad i \neq j .
\end{gathered}
$$

Here

$$
\left[\begin{array}{c}
n \\
m
\end{array}\right]_{q}=\frac{[n]_{q} !}{[n-m]_{q} ![m]_{q} !}
$$

and $[n]_{q} !=[n]_{q} \ldots[1]_{q},[n]_{q}=\frac{q^{n}-q^{-n}}{q-q^{-1}}, q_{i}=q^{d_{\imath}}$.

Remark 3.1. Over $\mathbf{C}[[q-1]]$ we can define elements

$$
h=\log q=\log (1+q-1)=\sum_{n \geq 1} \frac{(-1)^{n-1}}{n}(q-1)^{n}
$$

and

$$
H_{1}=\frac{1}{2 h d_{i}} \log \left(k_{i}^{2}\right)=\frac{1}{2 d_{i} h} \log \left(1+k_{i}^{2}-1\right) .
$$

We will also use notation

$$
q^{A}=\exp (h A)
$$

The algebra $U_{q}(\mathfrak{g})$ is a Hopf algebra [Ab] with the comultiplication

$$
\begin{aligned}
& \Delta\left(k_{i}\right)=k_{i} \otimes k_{i}, \\
& \Delta\left(e_{i}\right)=e_{i} \otimes k_{i}+1 \otimes e_{i}, \\
& \Delta\left(f_{i}\right)=f_{i} \otimes 1+k_{i}^{-1} \otimes f_{i} .
\end{aligned}
$$

From relations (3.1) and this form of the comultiplication it is easy to compute the action of the antipode on the generators:

$$
S\left(e_{i}\right)=-e_{i} k_{i}^{-1}, \quad S\left(f_{i}\right)=-k_{i} f_{i}, \quad S\left(k_{i}\right)=k_{i}^{-1} .
$$

The subalgebra of $U_{q}(\mathfrak{g})$ generated by $k_{i}$ is isomorphic to the universal enveloping algebra $U(\mathfrak{h})$ of a Cartan subalgebra $\mathfrak{h}$ of $\mathfrak{g}$ over $\mathbf{C}[[q-1]]$. We denote by $U_{q}\left(\mathfrak{b}_{+}\right)$ (respectively $U_{q}\left(\mathfrak{b}_{-}\right)$) the subalgebra of $U_{q}(\mathfrak{g})$ generated by $e_{i}$ and $k_{i}$ (respectively $f_{i}$ and $k_{i}$ ).

The remarkable property of $U_{q}(\mathfrak{g})$ is that it is a quasitriangular Hopf algebra, which means the following. 
Proposition 3.1 [Dr3]. There exists a distinguished invertible element $R \in U_{q}(\mathfrak{g}) \hat{\otimes}$ $U_{q}(\mathfrak{g})$ (here $\hat{\otimes}$ is a tensor product completed over $\left.\mathbf{C}[[q-1]]\right)$ which is called universal $R$-matrix and has the following properties:

$$
\begin{aligned}
\Delta^{\prime}(a) & =R \Delta(a) R^{-1}, \\
(\Delta \otimes \mathrm{id})(R) & =R_{13} R_{23}, \\
(\mathrm{id} \otimes \Delta)(R) & =R_{13} R_{12},
\end{aligned}
$$

where $\Delta^{\prime}(a)=\sigma \circ \Delta(a), \sigma$ is a permutation in $U_{q}(\mathfrak{g})^{\hat{\otimes} 2}(\sigma(x \otimes y)=y \otimes x)$ and $R_{12}=R \otimes 1, R_{23}=1 \otimes R, R_{13}=(\sigma \otimes \mathrm{id})\left(R_{23}\right)$ are elements of $U_{q}(\mathfrak{g})^{\hat{\otimes} 3}$.

This proposition follows from the double construction of the algebra $U_{q}(\mathfrak{g})$ [Dr3], (see also [RS1]): $U_{q}(\mathfrak{g})=D\left(U_{q}\left(\mathfrak{b}_{+}\right)\right) / U(\mathfrak{h})$, where $D(A)$ is a "quantum double" of a Hopf algebra. Using this description of $U_{q}(\mathfrak{g})$ it is easy to write a few first terms in the decomposition of $R$ as a power series in $e_{i}$ and $f_{i}$ :

$$
R=\exp \left(h \sum_{i, j=1}^{n}\left(B^{-1}\right)_{i j} H_{i} \otimes H_{j}\right)\left(1+\sum_{i=1}^{n}\left(1-e^{-h d_{\imath}}\right) e_{i} \otimes f_{\imath}+\ldots\right) .
$$

Here $B=\left(B_{i j}\right)$ is a symmetrized Cartan matrix: $B_{i j}=d_{i} a_{i j}$. It follows immediately from the double construction that $R=\sum_{i} a_{i} \otimes b_{i}$, where $a_{i} \in U_{q}\left(\mathfrak{b}_{+}\right)$and $b_{i} \in U_{q}\left(\mathfrak{b}_{-}\right)$. We did not write in (3.4) terms of the type $x \otimes y$, where $x \in U_{q}\left(\mathfrak{b}_{+}\right), y \in U_{q}\left(\mathfrak{b}_{-}\right)$are monomials of $e_{i}$ and $f_{i}$, respectively, of degree $(x)=\operatorname{degree}(y)>1$.

The explicit description of $R$ in terms of generators, $e_{i}, f_{i}, k_{i}$ can be found in [Ro2, KR2, LSo].

We recall a few standard definitions and facts related to a $U_{q}(\mathfrak{g})$-module $V$. A vector $v \in V$ is called a weight vector with weight $\lambda=\left(\lambda_{1}, \ldots, \lambda_{n}\right)$ if

$$
k_{i} v=q^{\lambda_{i}} v, \quad i=1, \ldots, l .
$$

A vector $v \in V$ is called primitive if

$$
e_{i} v=0, \quad i=1, \ldots, l \text {. }
$$

The $U_{q}(\mathfrak{g})$-module $V$ is called a highest weight module with the highest weight $\lambda$ if it is generated by a primitive vector $v_{\lambda} \in V$ of weight $\lambda$. The vector $v_{\lambda}$ is called the highest weight vector. Replacing $e_{i}$ by $f_{i}$ in (3.6) one can define a lowest weight module generated by a lowest weight vector $v_{\lambda}^{\prime}$.

Theorem 3.1 [L, Ro1]. i) any finite dimensional representation of $U_{q}(\mathfrak{g})$ is completely reducible. ii) An irreducible finite dimensional $U_{q}(\mathfrak{g})$-module is uniquely characterized by its highest weight and weight subspaces in such modules have the same dimensions as the corresponding weight subspaces in an irreducible $\mathfrak{g}$-module with the same highest weight.

We will need some further facts about the category of finite dimensional representations of $U_{q}(\mathfrak{g})$. Let us denote it as $\operatorname{Rep}_{q}(\mathfrak{g})$ and let us recall some basic properties of this category. As we will see, the category $\operatorname{Rep}_{q}(\mathfrak{g})$ is a tensor category (in the sense of Sect. 2, i.e. abelian braided monoidal category) with a certain additional structure.

First, the structure of the monoidal category on $\operatorname{Rep}_{q}(\mathfrak{g})$ is determined by the comultiplication in $U_{q}(\mathfrak{g})$. For, given two $U_{q}(\mathfrak{g})$-modules $V$ and $W$, define the representation $\pi_{V \otimes W}: U_{q}(\mathfrak{g}) \rightarrow \operatorname{End}(V \otimes W)$ as

$$
\pi_{V \otimes W}(a)=\left(\pi_{V} \otimes \pi_{W}\right)(\Delta(a)), \quad a \in U_{q}(\mathfrak{g}) .
$$


The associativity constraint $\varphi_{X, Y, Z}: X \otimes(Y \otimes Z) \rightarrow(X \otimes Y) \otimes Z$ is trivial because of the coassociativity of $U_{q}(\mathfrak{g})$. An identity $I$ object is by definition $\mathbf{C}$ considered as a trivial $U_{q}(\mathfrak{g})$-module and the natural transformations $\lambda_{X}, \varrho_{X}(2.45)$ are also trivial for any object $X$.

It follows from Proposition 3.1 that the category $\operatorname{Rep}_{q}(\mathfrak{g})$ is a braided monoidal category [JS] with the commutativity constraint $\gamma_{X, Y}: X \otimes Y \rightarrow Y \otimes X$ given by

$$
\gamma_{X, Y}=P^{X Y}\left(\pi_{X} \otimes \pi_{Y}\right)(R)
$$

where $P^{X Y}: X \otimes Y \rightarrow Y \otimes X$ is the permutation map $P^{X Y}(x \otimes y)=y \otimes x$. From the action of the comultiplication on the universal $R$-matrix we deduce the hexagon identities for $\gamma$ :

$$
\begin{aligned}
\gamma_{X \otimes Y, Z} & =\left(\gamma_{X, Z} \otimes \mathrm{id}_{Y}\right)\left(\mathrm{id}_{X} \otimes \gamma_{Y, Z}\right) \\
\gamma_{X, Y \otimes Z} & =\left(\mathrm{id}_{Y} \otimes \gamma_{X, Z}\right)\left(\gamma_{X, Y} \otimes \mathrm{id}_{Z}\right)
\end{aligned}
$$

Moreover, Theorem 3.1 implies that $\operatorname{Rep}_{q}(\mathfrak{g})$ is a semisimple abelian category with irreducible finite dimensional representations as simple objects.

Let us recall that a tensor category $\mathscr{C}$ is a rigid tensor category if for any object $X$ of the category $\mathscr{C}$ there exists an object $X^{*}$ and a pair of morphisms $\varepsilon_{X}: X^{*} \otimes X \rightarrow$ $I, \iota_{X}: I \rightarrow X \otimes X^{*}$ such that

$$
\lambda_{X^{*}}\left(\varepsilon_{X} \otimes \mathrm{id}\right)\left(\mathrm{id} \otimes \iota_{X}\right) \varrho_{X^{*}}^{-1}=\mathrm{id}_{X^{*}}, \quad \varrho_{X}\left(\mathrm{id} \otimes \varepsilon_{X}\right)\left(i_{X} \otimes \mathrm{id}\right) \lambda_{X}^{-1}=\mathrm{id}_{X} .
$$

The category $\operatorname{Rep}_{q}(\mathfrak{g})$ is a rigid tensor category. The module $V^{*}$ dual to $V$ is by definition a dual linear space to $V$ together with the following structure of $U_{q}(\mathfrak{g})$ module:

$$
(a \cdot l)(v)=l(S(a) \cdot v),
$$

where $S$ is the antipode for $U_{q}(\mathfrak{g}), l \in V^{*}, v \in V, a \in U_{q}(\mathfrak{g})$.

The next important property of the category $\operatorname{Rep}_{q}(\mathfrak{g})$ is balancing. In a balanced tensor category $\mathscr{C}$ we associate with each object $V \in \mathscr{C}$ an automorphism $\tau_{V}$ such that

$$
\tau_{X \otimes W}=\gamma_{V, W}^{-1} \gamma_{W, V}^{-1} \tau_{v} \otimes \tau_{W}, \quad \tau_{V^{*}}=\left(\tau_{V}\right)^{*}, \quad \tau_{I}=\mathrm{id} .
$$

For a tensor category $\mathscr{C}$ and an object $V \in \mathscr{C}$ define a morphism $v_{V}$ which is given by the following composition:

$$
v_{V}: V \stackrel{\text { id } \otimes \iota}{\longrightarrow} V \otimes V^{*} \otimes V^{* *} \stackrel{\gamma \otimes \text { id }}{\longrightarrow} V^{*} \otimes V \otimes V^{* *} \stackrel{\varepsilon \otimes \text { id }}{\longrightarrow} V^{* *} .
$$

This is an isomorphism with $v_{V}^{-1}$ given by the composition

$$
v_{V}^{-1}: V^{* *} \stackrel{\mathrm{id} \otimes \iota}{\longrightarrow} V^{* *} \otimes V \otimes V^{*} \stackrel{\mathrm{id} \otimes \gamma^{-1}}{\longrightarrow} V^{* *} \otimes V^{*} \otimes V \stackrel{\varepsilon \otimes \mathrm{id}}{\longrightarrow} V .
$$

A remarkable property of balanced categories is that the isomorphisms

$$
\delta_{V}: V \rightarrow V^{* *}, \quad \delta_{V}=v_{V} \circ \tau_{V}^{-1}
$$

are functorial [KaR]: $\delta_{V \otimes W}=\delta_{V} \otimes \delta_{W}, \delta_{I}=\mathrm{id},\left(\delta_{X^{*}}\right)^{-1}=\left(\delta_{X}\right)^{*}$.

Following [RT] we will say that a quasitriangular Hopf algebra $A$ is a ribbon Hopf algebra if there exists a central element $v \in A$ such that

$$
\Delta(v)=\left(R_{21} R_{12}\right)^{-1} v \otimes v, \quad S(v)=v .
$$

Here $R_{21}=\sigma(R), \sigma(a \otimes b)=b \otimes a$. The category of representations of any ribbon Hopf algebra is a balanced tensor category. 
The algebra $U_{q}(\mathfrak{g})$ is a ribbon Hopf algebra [D1] with

$$
v=u q^{-2 \varrho}
$$

where, if we write $R=\sum_{i} a_{i} \otimes b_{i}$ for the universal $R$-matrix, $u=\sum_{i} S\left(b_{i}\right) a_{i}$; and $\varrho$ is half sum of positive roots. For an irreducible representation $V$ we have:

$$
\pi_{\lambda}(v)=q^{C(\lambda)} \cdot I_{V}
$$

where $C(\lambda)=(\lambda, \lambda+2 \varrho)$ and $(\lambda, \mu)$ is the bilinear form introduced in Sect. 2. The functorial isomorphism $\delta_{V}: V \rightarrow V^{* *}$ is given by the map $a \mapsto q^{2} \varrho a$.

The functoriality of $\delta_{V}$ implies the following relations for $R^{V W}=\left(\pi_{V} \otimes \pi_{W}\right)(R)$ $[\mathrm{R}]$,

$$
\left(\left(\left(\left(R^{V W}\right)^{t_{1}}\right)^{-1}\right)^{t_{1}}\right)^{-1}=\left(q^{-2 \varrho} \otimes I_{W}\right) R^{V W}\left(q^{2 \varrho} \otimes I_{W}\right)
$$

and

$$
\left(q^{2 \varrho} \otimes q^{2 \varrho}\right) R^{V W}=R^{V W}\left(q^{2 \varrho} \otimes q^{2 \varrho}\right) .
$$

For a finite dimensional $U_{q}(\mathfrak{g})$-module $V$ we denote by $L^{ \pm, V}$ the following elements of $U_{q}(\mathfrak{g}) \otimes \operatorname{End}(V)$ :

$$
L^{+, V}=\left(\mathrm{id} \otimes \pi_{V}\right)\left(R_{21}\right), \quad L^{-, V}=\left(\mathrm{id} \otimes \pi_{V}\right)\left(R^{-1}\right) .
$$

If we fix a basis $\left\{e_{i}\right\}$ in $V$, we can regard $L^{ \pm, V}$ as matrices with matrix elements $L_{i j}^{ \pm, V}$ being elements of $U_{q}(\mathfrak{g})$. From the Yang-Baxter equation for $R$ we get relations between $L^{ \pm, V}$ :

$$
\begin{aligned}
R^{V W} L_{1}^{+, V} L_{2}^{+, W} & =L_{2}^{+, W} L_{1}^{+, V} R^{V W} \\
R^{V W} L_{1}^{+, W} L_{2}^{-, W} & =L_{2}^{-, W} L_{1}^{+, V} R^{V W} \\
R^{V W} L_{1}^{-, V} L_{2}^{-, W} & =L_{2}^{-, W} L_{1}^{-, V} R^{V W}
\end{aligned}
$$

By $L_{1}^{ \pm, V}, L_{2}^{ \pm, W}$ we understand the following matrices in $V \otimes W$ :

$$
L_{1}^{ \pm, V}=L^{ \pm, V} \otimes I_{W}, \quad L_{2}^{ \pm, W}=I_{V} \otimes L^{ \pm, W}
$$

where $I_{X}$ is the unit matrix in $X$. From the quasitriangularity of $U_{q}(\mathfrak{g})$ we can obtain the action of the comultiplication on matrices $L^{ \pm, V}$ :

$$
\Delta^{\prime}\left(L_{i j}^{ \pm, V}\right)=\sum_{k} L_{i k}^{ \pm, V} \otimes L_{k j}^{ \pm, V}
$$

Proposition. For any finite dimensional representation $V$ matrix elements $L_{i j}^{ \pm, V}$ generate $U_{q}(\mathfrak{g})$ (over $\left.\mathbf{C}[[q-1]]\right)$, such that $L_{i j}^{ \pm, V}$ generates $U_{q}\left(\mathfrak{b}_{\mp}\right) \hookrightarrow U_{q}(\mathfrak{g})$.

Let $\left(\Phi^{\lambda}\right)_{\nu}^{\mu}$ be an intertwiner

$$
\left(\Phi^{\lambda}\right)_{\nu}^{\mu}: V_{\nu} \rightarrow V_{\mu} \otimes V_{\lambda}
$$

When $V$ is $V_{\lambda}$ we will often write $\lambda$ instead of $V_{\lambda}$ in various indexes, e.g. $L^{ \pm, \lambda}$ instead of $L^{ \pm, V_{\lambda}}$. The following result will be important for the future.

Theorem 3.3. Let $e_{i}$ be a basis in $V_{\lambda}$ and $\left(\Phi^{\lambda}\right)_{i}$ be a corresponding map $V_{\nu} \rightarrow V_{\mu}$, then

$$
\sum_{j, k=1}^{\operatorname{dim}\left(V_{\lambda}\right)}\left(L^{+, \lambda}\right)_{i j}\left(\Phi^{\lambda}\right)_{k}\left(q^{2 \varrho}\right)_{k k} S\left(\left(L^{-, \lambda}\right)_{j k}\right)=q^{C(\nu)-C(\mu)}\left(\Phi^{\lambda}\right)_{i},
$$

where $C(\lambda)$ is the value of $\mathfrak{g}$-Casimir elements on $V_{\lambda}$ as in (3.11). 
Proof. First let us prove the following lemma.

Lemma 3.1. The map $\sum_{j, k}\left(L^{+, \lambda}\right)_{i j}\left(\Phi^{\lambda}\right)_{k}\left(q^{2 \varrho}\right)_{k k} S\left(L^{-, \lambda}\right)_{j k}: V_{\nu} \rightarrow V_{\mu}$ coincides with the map $A_{i}$, where $A_{i}$ is a component of the composition map

$$
\begin{aligned}
A: V_{\nu} & \stackrel{\mathrm{id} \otimes \iota_{\lambda}}{\longrightarrow} V_{\nu} \otimes V_{\lambda} \otimes\left(V_{\lambda}\right)^{*} \stackrel{\gamma_{\nu \lambda} \otimes \mathrm{id}}{\longrightarrow} V_{\lambda} \otimes V_{\nu} \otimes\left(V_{\lambda}\right)^{*} \\
& \stackrel{\mathrm{id} \otimes \Phi \otimes \mathrm{id}}{\longrightarrow} V_{\lambda} \otimes V_{\mu} \otimes V_{\lambda} \otimes\left(V_{\lambda}\right)^{*} \stackrel{\mathrm{id} \otimes \mathrm{id} \otimes q^{2} \varrho_{\mathrm{id}}}{\longrightarrow} V_{\lambda} \otimes V_{\mu} \otimes V_{\lambda}^{* *} \otimes V_{\lambda}^{*} \\
& \stackrel{\mathrm{id} \otimes \mathrm{id} \otimes \varepsilon_{\lambda^{*}}}{\longrightarrow} V_{\lambda} \otimes V_{\mu} \stackrel{\gamma_{\lambda \mu}}{\longrightarrow} V_{\mu} \otimes V_{\lambda},
\end{aligned}
$$

where the morphisms $\iota_{\lambda}, \gamma_{\lambda \mu}, \Phi, q^{2 \varrho}, \varepsilon_{\lambda}$ are as above.

To prove the lemma it is enough to compute the action of the composition map and to compare it with the map in (3.18). The computation gives

$$
A e_{u}^{\nu}=\sum\left(\pi_{\lambda}\left(a_{j} b_{k}\right)\right)_{i}^{l}\left(\pi_{\mu}\left(b_{j}\right)\right)_{s}^{t}(\Phi)_{p}^{s v}\left(\pi_{\nu}\left(a_{k}\right)\right)_{u}^{p} \cdot\left(\pi_{\lambda}\left(q^{2 \varrho}\right)\right)_{v}^{i} \cdot\left(e_{t}^{\mu} \otimes e_{l}^{\lambda}\right) .
$$

Here we use matrix notation $x e_{i}=\sum_{j} x_{i}^{j} e_{j}$, and $a_{i}, b_{i}$ are components of the universal $R$-matrix: $R=\sum_{i} a_{i} \otimes b_{i}$. From the definition of $L^{ \pm, \lambda}$ we conclude that (3.19) coincides with the left-hand side of (3.18).

Now, using the identities of a tensor category, the functoriality of the morphism $q^{2 \varrho}$ in (3.19) and the balancing property of the category $\operatorname{Rep}_{q}(\mathfrak{g})$ one can show that

$$
A=q^{C(\nu)-C(\mu)} \Phi .
$$

The simplest way to do it is to use the "graphic representation" [Re1] of the category $\mathscr{C}$. As it was shown in [RT] there exists a functor from the category of framed graphs to the category $\operatorname{Rep}_{q}(\mathfrak{g})$. The identity (3.21) corresponds to the following isotopy of framed graphs:

Fig. 1
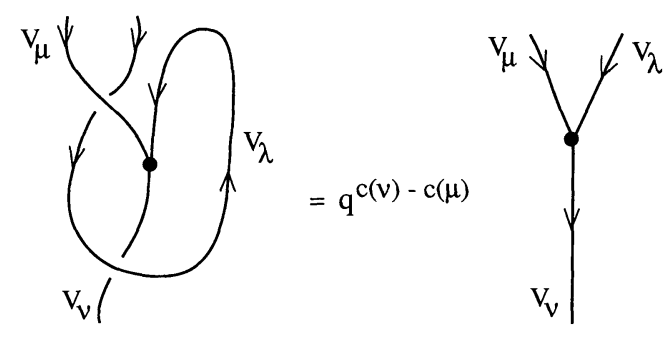

Finally we would like to note that in Sects. 2 and 3 we have studied two classes of tensor categories namely $\operatorname{Mon}_{k}(\hat{\mathfrak{g}})$ and $\operatorname{Rep}\left(U_{q}(\mathfrak{g})\right)$. The following deep theorem establishes an explicit relation between them.

Theorem 3.3 [D2]. For $q=\exp (2 \pi i /(k+g))$ and $k \notin \mathbf{Q}$ there is an equivalence of tensor categories

$$
\operatorname{Rep}\left(U_{q}(\mathfrak{g})\right) \cong \operatorname{Mon}_{k}(\hat{\mathfrak{g}}) .
$$

We will not use this result in the present paper. However in the subsequent Sects. 4,5 , and 6 we will study the quantum analogues of the above (pre-)tensor categories. 
The statement of Theorem 3.3 will suggest the corresponding conjectures which we will state at the end of Sect. 6 .

\section{Quantum Affine Lie Algebras}

Let $\hat{\mathfrak{g}}$ be an affine Lie algebra associated to $\mathfrak{g}$ with extended Cartan matrix $\hat{A}=\left(a_{i j}\right)$, $i, j=0, \ldots, l=\operatorname{rank} \mathfrak{g}$ and let $q$ be a formal variable.

Definition 4.1 [Dr1, J2]. The algebra $U_{q}(\hat{\mathfrak{g}})$ is an associative algebra over the ring of formal power series $\mathbf{C}[[q-1]]$ with generators $k_{i}, e_{i}, f_{i}, i=0,1, \ldots, l$, and with relations (3.1) between them.

Definition 4.2. The algebra $U_{q}(\tilde{\mathfrak{g}})$ is an algebra over $\mathbf{C}[[q-1]]$ with generators $e_{i}$, $k_{i}, f_{i}, i=0, \ldots, l ; d$ and with relations (3.1) and

$$
\left[d, k_{i}\right]=0, \quad\left[d, e_{i}\right]=\delta_{i, 0} e_{i}, \quad\left[d, f_{i}\right]=-\delta_{i, 0} f_{i} .
$$

Algebras $U_{q}(\hat{\mathfrak{g}})$ and $U_{q}(\tilde{\mathfrak{g}})$ are Hopf algebras with the comultiplication (3.2) and

$$
\Delta(d)=d \otimes 1+1 \otimes d
$$

Let $z$ be a formal variable. Define an automorphism $D_{z}$ of $U_{q}(\tilde{\mathfrak{g}}) \otimes \mathbf{C}\left[z, z^{-1}\right]$ as

$$
\begin{gathered}
D_{z}\left(e_{i}\right)=z^{\delta_{i, 0}} e_{i}, \quad D_{z}\left(f_{i}\right)=z^{-\delta_{i, 0}} f_{i} \\
D_{z}\left(k_{i}\right)=k_{i}, \quad D_{z}(d)=d
\end{gathered}
$$

and define maps

$$
\begin{gathered}
\Delta_{z}, \Delta_{z}^{\prime}: U_{q}(\hat{\mathfrak{g}}) \otimes \mathbf{C}\left[z, z^{-1}\right] \rightarrow U_{q}(\hat{\mathfrak{g}})^{\otimes 2} \otimes \mathbf{C}\left[z, z^{-1}\right], \\
\Delta_{z}(a)=\left(D_{z} \otimes \mathrm{id}\right) \Delta(a), \quad \Delta_{z}^{\prime}(a)=\left(D_{z} \otimes \mathrm{id}\right) \Delta^{\prime}(a),
\end{gathered}
$$

where $\Delta^{\prime}(a)=\sigma \circ \Delta(a), \sigma(a \otimes b)=b \otimes a$.

Drinfeld's construction of universal $R$-matrix that we used in Sect. 3 (Proposition 3.1) can be generalized to an arbitrary Kac-Moody algebra [Dr3, Sect. 13], in particular, to the affine case. We denote by $\mathscr{B}$ the universal $R$-matrix for $U_{q}(\tilde{\mathfrak{g}})$. It follows from the Drinfeld construction that

$$
\mathscr{B} \in U_{q}\left(\tilde{\mathfrak{b}}_{+}\right) \hat{\otimes} U_{q}\left(\tilde{\mathfrak{b}}_{-}\right) \mapsto U_{q}(\tilde{\mathfrak{g}}) \hat{\otimes} U_{q}(\tilde{\mathfrak{g}}) .
$$

For any two finite-dimensional representations $V$ and $W$ of $U_{q}(\hat{\mathfrak{g}})$ we would like to define an operator $\mathscr{R}^{V W}$ as a projection of $\mathscr{R}$ acting in $V \otimes W$ as in Sect. 3. This cannot be done in a straightforward way for two closely related reasons. First is that finite dimensional representations are in fact representations of $U_{q}(\hat{\mathfrak{g}})$ and not $U_{q}(\tilde{\mathfrak{g}})$, second is that if we would "gauge out" the element $d$ the projection of $\mathscr{B}$ still would be meaningless as observed in [Dr3]. We will resolve this problem in two steps. First we define a universal $R$-matrix $\mathscr{B}(z)$ for $U_{q}(\tilde{\mathfrak{g}})$ depending on a formal parameter $z$ by the formula

$$
\mathscr{R}(z)=\left(D_{z} \otimes \mathrm{id}\right)(\mathscr{R})
$$

Then the Drinfeld construction implies that following. 
Proposition 4.1. (1) There exist unique elements $\mathscr{B}(z) \in U_{q}\left(\tilde{\mathfrak{b}}_{+}\right) \hat{\otimes} U_{q}\left(\tilde{\mathfrak{b}}_{-}\right) \otimes \mathbf{C}[[z]] \mapsto$ $U_{q}\left(\tilde{\mathfrak{g}}^{\hat{\otimes} 2} \otimes \mathbf{C}[[z]]\right.$, where $\hat{\otimes}$ is the completion of $\otimes$ over $\mathbf{C}[[q-1]]$ such that

$$
\begin{aligned}
\mathscr{B}(z) \Delta_{z}(a) & =\Delta_{z}^{\prime}(a) \mathscr{B}(z), \\
\left(\Delta_{z} \otimes \mathrm{id}\right)(\mathscr{R}(w)) & =\mathscr{R}_{13}(z w) \mathscr{B}_{23}(w), \\
\left(\mathrm{id} \otimes \Delta_{w}\right)(\mathscr{R}(z w)) & =\mathscr{R}_{13}(z) \mathscr{R}_{12}(z w) .
\end{aligned}
$$

Here

$$
\begin{aligned}
& \mathscr{B}_{12}(z)=\mathscr{B}(z) \otimes 1 \in U_{q}(\tilde{\mathfrak{g}})^{\hat{\otimes} 3} \otimes \mathbf{C}[[z]], \\
& \mathscr{B}_{13}(z)=1 \otimes \mathscr{B}(z) \in U_{q}(\tilde{\mathfrak{g}})^{\otimes 3} \otimes \mathbf{C}[[z]], \\
& \mathscr{B}_{23}(z)=(\sigma \otimes \mathrm{id})\left(\mathscr{B}_{13}(z)\right) \in U_{q}(\tilde{\mathfrak{g}})^{\otimes 3} \otimes \mathbf{C}[[z]] .
\end{aligned}
$$

(2) The element $\mathscr{R}(z)$ has the followimg form:

$$
\begin{aligned}
\mathscr{B}(z)= & \exp (h)\left((c \otimes d+d \otimes c)+\sum_{i, j=1}^{l}\left(B^{-1}\right)_{i j} H_{i} \otimes H_{j}\right) \\
& \times\left(1+\sum_{i=1}^{l} 2 \operatorname{sh}\left(\frac{h d_{i}}{2}\right) e_{i} \otimes f_{i}+2 z \operatorname{sh}\left(\frac{h}{2}\right) e_{0} \otimes f_{0}+\ldots\right) .
\end{aligned}
$$

Here $d_{i}=a_{i}^{\vee} / a_{i}$ for $i=1, \ldots, l, B_{i j}=d_{i} a_{i j}, i, j=1, \ldots, l ; a_{i}, a_{i}^{\vee}$ are labels of the Dynkin diagrams of Lie algebras $\mathfrak{g}$ and $\mathfrak{g}^{\vee}$ (with dual root system) respectively; $c=H_{0}+H_{\theta}$ where $\theta$ is a maximal root in $\mathfrak{g}$. We omit the higher order terms over $z$, $q-1$ and $e_{i}, f_{i}$. All these higher order terms do not depend on $d$.

The proof is based on the double construction for $U_{q}(\tilde{\mathfrak{g}})$ and is parallel to the proof of Proposition 3.1 [Dr3]. We omit the details of the arguments.

An important corollary of Proposition 4.1 is the Yang-Baxter equation with spectral parameter (cf. $[\mathrm{F}])$ for the element $\mathscr{R}(z)$ :

$$
\mathscr{B}_{12}(z) \mathscr{B}_{13}(z w) \mathscr{B}_{23}(w)=\mathscr{B}_{23}(w) \mathscr{B}_{13}(z w) \mathscr{B}_{12}(z) .
$$

All factors in this equation are elements of $U_{q}(\tilde{\mathfrak{g}})^{\otimes} \hat{\otimes}^{3} \otimes \mathbf{C}[[z]] \otimes \mathbf{C}[[w]]$. We will call $\mathscr{B}(z)$ the universal $R$-matrix for $U_{q}(\tilde{\mathfrak{g}})$.

Since the dependence of the element $\mathscr{B}(z)$ on $d$ is explicitly given by (4.6), we can define the universal $R$-matrix for $U_{q}(\tilde{\mathfrak{g}})$ as

$$
R(z)=\exp (-h(c \otimes d+d \otimes c) \mathscr{B}(z) .
$$

Relations (4.5) give the following properties of $\mathscr{B}(z)$ :

$$
\begin{aligned}
R(z) \Delta_{z}(a) & =\left(D_{q^{c_{1}}}^{-1} \otimes D_{q^{c_{1}}}^{-1}\right)\left(\Delta_{z}^{\prime}(a)\right) \cdot R(z), \\
(\Delta \otimes \mathrm{id})(R(w)) & =R_{13}\left(w q^{c_{2}}\right) R_{23}(w), \\
(\mathrm{id} \otimes \Delta)(R(w)) & =R_{13}\left(w q ^ { c _ { 2 } } \left(R_{12}(w),\right.\right. \\
R_{12}(z) R_{13}\left(z w q^{c_{2}}\right) R_{23}(w) & =R_{23}(w) R_{13}\left(z w q^{-c_{2}}\right) R_{12}(z) .
\end{aligned}
$$

Here $c_{1}=c \otimes 1, c_{2}=1 \otimes c$ in the first relation and $c_{2}=1 \otimes c \otimes 1$ in others.

Now, for any two finite dimensional representations $V$ and $W$ we can define an operator

$$
R^{V W}(z)=\left(\pi_{V} \otimes \pi_{W}\right)(R(z))
$$


as in Sect. 3. Since for any finite dimensional representation $V \pi_{V}(c)=0$, the operators of the type (4.8) satisfy the Yang-Baxter equation with argument as in (4.7).

Let $V$ be a finite dimensional $U_{q}(\hat{\mathfrak{g}})$-module with $\pi_{V}: U_{q}(\hat{\mathfrak{g}}) \rightarrow \operatorname{End}(V)$, and let $D_{z}$ be the automorphism (4.3) of $U_{q}(\hat{\mathfrak{g}}) \otimes \mathbf{C}\left[z, z^{-1}\right]$. We define a new representation

$$
\pi_{V(z)}: U_{q}(\hat{\mathfrak{g}}) \rightarrow \operatorname{End}(V) \otimes \mathbf{C}\left[z, z^{-1}\right]
$$

by the formula $\pi_{V(z)}(a)=\pi_{V}\left(D_{z}(a)\right), a \in U_{q}(\hat{\mathfrak{g}})$. Spezialising $z$ to a complex number we get a one-parameter family of finite dimensional modules $V(z)$ connected by the action of the automorphism (4.3): $\pi_{V(z w)}(a)=\pi_{V(z)}\left(D_{W}(a)\right)$. The definition (4.11) and the first equation in (4.9) also imply that

$$
R^{V W}(z) \pi_{V(z w) \otimes W(w)}(\Delta(a))=\pi_{V(z w) \otimes W(w)}\left(\Delta^{\prime}(a)\right) R^{V W}(z) .
$$

As in Sect. 3, for any module $V$ we define a right dual module $V^{*}$ as a linear space which is dual to $V$ and has the following structure of $U_{q}(\hat{\mathfrak{g}})$-module:

$$
\left\langle a v^{\prime}, v\right\rangle=\left\langle v^{\prime}, S(a) v\right\rangle, \quad v^{\prime} \in V^{*}, \quad v \in V .
$$

Proposition 4.2. $V(z)^{* *} \stackrel{\sim}{\rightarrow} V\left(z q^{g}\right), v \mapsto q^{-2 \varrho} v$, where $g$ is a dual Coxeter number.

Proof. To prove this proposition, it is enough to compute the action of the square of antipode:

$$
S^{2}(a)=q^{2 \varrho} D_{q^{g}}(a) q^{-2 \varrho} .
$$

The latter can be checked easily on generators.

\section{Proposition 4.3.}

$$
(S \otimes \mathrm{id})(R(z))=R(z)^{-1}, \quad\left(\mathrm{id} \otimes S^{-1}\right)(R(z))=R(z)^{-1} .
$$

Proof. This proposition follows from the action of the comultiplication on $R(z)$ from the property of the antipode: $m(S \otimes \mathrm{id}) \Delta=m(\mathrm{id} \otimes S) \Delta=\iota \varepsilon$ and from the identity $(\varepsilon \otimes \mathrm{id})(R(z))=1=(\mathrm{id} \otimes \varepsilon) R(z)$, which is a corollary of (4.5) and the identity $(\varepsilon \otimes \mathrm{id}) \Delta=(\mathrm{id} \otimes \varepsilon) \Delta=\mathrm{id}$. Here $\varepsilon$ is a counit and $m$ is a multiplication for $U_{q}(\tilde{\mathfrak{g}})$.

\section{Proposition 4.4.}

$$
R^{V^{*}, W}(z)=\left(R^{V W}(z)^{-1}\right)^{t_{1}}
$$

where $t_{1}$ is a transposition over the first space.

Proof. Let

$$
R(z)=\sum_{n \geq 0} \sum_{i} a_{i}(n) \otimes b_{i}(n) z^{n} .
$$

For $R^{V^{*}, W}(z)$, we have:

$$
R^{V^{*}, W}(z)=\left(\left(\pi_{V} \otimes \pi_{W}\right)((S \otimes \mathrm{id}) R(z))\right)^{t_{1}} .
$$

Now the statement follows from Proposition 4.3. Similarly to the right dual $V^{*}$, we can define a left dual module ${ }^{*} V$ as a dual linear space to $V$ with the following $U_{q}(\hat{\mathfrak{g}})$-module structure:

$$
\left\langle a v^{\prime}, v\right\rangle=\left\langle v^{\prime}, S^{-1}(a) v\right\rangle, \quad v^{\prime} \in^{*} V, v \in V .
$$

Proposition 4.5. (1) ${ }^{* *} W(z) \stackrel{\sim}{\rightarrow} W\left(z q^{-g}\right), v \rightarrow q^{2 \varrho} v$.

(2) $R^{V,{ }^{*} W}(z)=\left(R^{V W}(z)^{-1}\right)^{t_{2}}$. 
The proof is similar to Propositions 4.2 and 4.4.

Proposition 4.6. $R^{V^{*}, W^{*}}(z)=\left(R^{V W}(z)\right)^{t_{1} t_{2}}$.

Proof. This proposition follows from the definition of $V^{*}$ and from Proposition 4.3.

Theorem 4.1. For any pair of finite dimensional $U_{q}(\hat{\mathfrak{g}})$-modules, we have:

$$
\begin{gathered}
\left(\left(\left(R^{V W}(z)^{-1}\right)^{t_{1}}\right)^{-1}\right)^{t_{1}}=\left(\pi_{V}\left(q^{2 \varrho}\right) \otimes 1_{W}\right) R^{V W}\left(z q^{2 g}\right)\left(\pi_{V}\left(q^{-2 \varrho}\right) \otimes 1_{W}\right), \\
\left(\left(\left(R^{V W}(z)^{-1}\right)^{t_{2}}\right)^{-1}\right)^{t_{2}}=\left(1_{V} \otimes \pi_{W}\left(q^{-2 \varrho}\right)\right) R^{V W}\left(z q^{-2 g}\right)\left(1_{V} \otimes \pi_{W}\left(q^{2 \varrho}\right)\right) .
\end{gathered}
$$

Proof. The theorem follows from Propositions 4.2-4.5. The first equality is a representation of $R^{V^{* *}, W}(z)$. The second one is the representation for $R^{V, * *}(z)$.

Theorem 4.2. Let $V$ and $W$ be two finite dimensional irreducible $U_{q}(\hat{\mathfrak{g}})$-modules.

(1) The operator $R^{V W}(z)$ has the following presentation:

$$
R^{V W}(z)=f_{V W}(z) Q^{V W}(z)
$$

where $Q^{V W}(z)$ is a matrix polynomial over $z$ without common zeros. The function $f_{V W}(z)$ is a meromorphic function on $\mathbf{C}$ such that $f_{V W}(0)=1$ and $f_{V W}(z) \sim$ $z^{-p(V, W)}$, where $p(V, W)$ is the degree of the polynomial $Q^{V, W}(z)$. This representation is unique.

(2) The operator $R^{V W}(z)$ satisfies the following unitarity condition

$$
R^{V W}(z) R_{21}^{W V}\left(z^{-1}\right)=I_{V \otimes W} .
$$

Proof. (1) By the definition of $R^{V W}(z)$ and of $R(z)$ the map $P^{V W} R^{V W}(z)$ is an intertwining operator: $V(z) \otimes W \rightarrow W \otimes V(z), W \equiv W(1)$. If $z$ is a formal variable, modules $V(z) \otimes W$ and $W \otimes V(z)$ are irreducible. Therefore, such an intertwining operator is unique up to a scalar factor.

Therefore, up to this factor, it is defined by the system of linear equations

$$
R^{V W}(z)\left(\pi_{V} \otimes \pi_{W}\right)\left(D_{z} \otimes \mathrm{id}\right)(\Delta(a))=\left(\pi_{V} \otimes \pi_{W}\right)\left(D_{z} \otimes \mathrm{id}\right)\left(\Delta^{\prime}(a)\right) \cdot R^{V W}(z)
$$

for $a=k_{i}, e_{i}, f_{i}, i=0, \ldots, l$. Since this equation is linear over $z, z^{-1}$ we have a factorized representation (4.19), where polynomial $Q^{V W}(z)$ is fixed uniquely up to the constant. Fix this constant by the condition $f_{V W}(0)=1$.

Let us prove that the formal power series $f_{V W}(z)$ has an analytic continuation.

Consider intertwiners $V^{* *}(z) \otimes W \rightarrow W \otimes V^{* *}(z)$. Since $z$ is a formal variable both of these representations are irreducible and therefore all such intertwining operators differ up to a scalar multiplier. From the definition of $V^{* *}$ we have that $P^{V^{* *}, W}\left(\left(\left(Q^{V W}(z)^{-1}\right)^{t_{1}}\right)^{-1}\right)^{t_{1}}$ is one of them. The isomorphism $V^{* *}(z) \cong V\left(z q^{2 g}\right)$ implies that $P^{V^{* *}, W} \cdot\left(\pi_{V}\left(q^{2 \varrho}\right) \otimes Q^{V W}\left(z q^{2 g}\right)\left(\pi_{V}\left(q^{-2 \varrho}\right) \otimes I_{W}\right)\right.$ is also an intertwining operator $V^{* *}(z) \otimes W \rightarrow W \otimes V^{* *}(z)$. Therefore, there exists rational functions $r_{V W}(z)$ such that

$$
\left(\left(\left(Q^{V W}(z)^{-1}\right)^{t_{1}}\right)^{-1}\right)^{t_{1}}=r_{V W}(z)\left(\pi_{V}\left(q^{2 \varrho}\right) \otimes 1_{W}\right) Q^{V W}\left(z q^{2 g}\right) \cdot\left(\pi_{V}\left(q^{-2 \varrho}\right) \otimes 1_{W}\right) .
$$

Since $Q^{V W}(z)$ is a polynomial the function $r_{V W}(z)$ must be a rational one. If $p(V, W)$ is a degree of the polynomial $Q_{V W}(z)$, we have for the rational function $r_{V W}(z)$,

$$
r_{V W}(0)=1, \quad r_{V W}(z) \cong q^{-p(V, W) 2 g}, \quad z \rightarrow \infty .
$$

From the definition of $Q^{V W}$, we have also

$$
r_{V W}(z)=1 \bmod (q-1) .
$$


Comparing (4.22) with (4.17), we obtain difference equations for $f_{V W}(z)$ :

$$
f_{V W}\left(z q^{2 g}\right)=r_{V W}(z) f_{V W}(z)
$$

with the condition $f_{V W}(0)=1$.

Let

$$
r_{V W}(z)=\prod_{j=1}^{p(V, W)} \frac{1-q^{\alpha_{\jmath}} z}{1-q^{\beta_{j}} z}
$$

where $\sum_{j=1}^{p(V, W)}\left(\alpha_{j}-\beta_{j}\right)=2 g p(V, W)$ and $\alpha_{j}, \beta_{j} \in \mathbb{C}$.

Proposition 4.7. Equation (4.25) with the function $r_{V W}(z)$ given by (4.26) has a unique solution over $\mathbf{C}[[z]] \otimes \mathbf{C}[[q-1]]$. It has the following form:

$$
f_{V W}(x)=\prod_{j=1}^{p(V, W)} \frac{\left(z q^{\alpha_{j}} ; q^{2 g}\right)_{\infty}}{\left(z q^{\beta_{j}} ; q^{2 g}\right)_{\infty}}
$$

where

$$
(z ; q)_{\infty}=\prod_{n \geq 0}\left(1-z q^{n}\right) .
$$

The proof is a straightforward computation. Uniqueness is obvious over $\mathbf{C}[[z]]$.

It is clear that we can continue the solution (4.27) from $\mathbf{C}[[q-1]]$ to complex values of $q$ such that $|q|<1$. Then we can continue the result from power series over $z$ to complex values of $z$. As a result, we obtain meromorphic functions of $z$ (for $|q|<1)$ such that it satisfies (4.24) and $f_{V W}(z) \cong z^{-p(V, W)}$, where $|z| \rightarrow \infty$.

(2) Now the equality (4.20) makes sense. Moreover, from the irreducibility of $V(z) \otimes$ $W$ (we assume now that $z, q \in \mathbb{C}, z \neq q^{n}, n \in \mathbf{Z}$ ) we conclude that

$$
R_{12}^{V W}(z) R_{21}^{W V}\left(z^{-1}\right)=g_{V W}(z) \cdot 1_{V \otimes W}
$$

for some scalar function $g_{V W}(z)$. Or equivalently,

$$
R^{V W}(z)=g_{V W}(z)\left(R_{21}^{W V}\left(z^{-1}\right)\right)^{-1}
$$

If we substitute this equality to the first relation from Theorem 4.1, we get:

$$
\frac{g_{V W}(z)}{g_{V W}\left(z q^{2 g}\right)}\left(\left(R_{21}^{W V}\left(z^{-1}\right)^{t_{1}}\right)^{-1}\right)^{t_{1}}=\pi_{V}\left(q^{2 \varrho}\right) R_{21}^{W V}\left(z^{-1} q^{-2 g}\right)^{-1} \pi_{V}\left(g^{-2 \varrho}\right)
$$

or

$$
\frac{g_{V W}(z)}{g_{V W}\left(z q^{2 g}\right)}\left(\left(\left(R_{12}^{V W}\left(z^{-1}\right)^{t_{2}}\right)^{-1}\right)^{t_{2}}\right)^{-1}=\pi_{W}\left(q^{-2 \varrho}\right) R_{12}^{V W}\left(z^{-1} q^{-2 g}\right) \pi_{W}\left(g^{2 \varrho}\right) .
$$

Now from the second relation of Theorem 4.1, we conclude that

$$
g_{V W}\left(z q^{2 g}\right)=g_{V W}(z) \text {. }
$$

Lemma 4.2. $g_{V W}(0)=1$.

Proof. The definition of $R(z)$ implies that $R^{V W}(0)=\bar{R}^{V W}$, where $\bar{R}^{V W}$ is the $U_{q}(\mathfrak{g})$ $R$-matrix in $V \otimes W$ regarded as $U_{q}(\mathfrak{g})$-module. From the relation (4.28) we conclude that $R^{V W}(\infty)=g_{V W}\left(\bar{R}_{21}^{W V}\right)^{-1}$ for some $g_{V W} \equiv g_{V W}(0)$. The definition and the properties of $R^{V W}(z)$ imply that the morphisms $P^{V W} R^{V W}(\infty): V \otimes W \rightarrow W \otimes V$ 
satisfy the hexagon identities (3.9) in the category $\operatorname{Rep}_{q}(\mathfrak{g})$ as well as other identities for the commutativitiy constraint $\gamma_{V, W}$ (3.8). Therefore $g_{V W}$ is such that $g_{V \otimes W, U}=$ $g_{V, U} g_{W, U}, g_{V, U \otimes W}=g_{V, U} g_{V, W}$, and $g_{V^{*}, W}=g_{V, W}^{-1}=g_{V, W^{*}}$. The category $\operatorname{Rep}_{q}(\mathfrak{g})$ is a semisimple tensor category, i.e. any representation can be obtained as a summand of tensor product of fundamental representations (for most types of $\mathfrak{g}$ it is sufficient to consider just one fundamental representation). The above identities imply algebraic equations for $g_{V, W}$ for fundamental irreducible representations $V$ and $W$. Because of this and the condition $g_{V W}=1 \bmod (q-1)$ we conclude that $g_{V W}=1$.

Since there is only one solution to (4.29), with the condition $g_{V W}(0)=1$, we have $g_{V W}(z)=1$, which ends the proof of the theorem.

Remark 4.1. Let $v$ and $w$ be $U_{q}(\mathfrak{g})$-highest weight vectors in $V$ and $W$ respectively. Since $Q^{V W}(z)$ is an intertwining operator

$$
Q^{V W}(z) v \otimes w=p_{V W}(z) v \otimes w
$$

where $p_{V W}(z)$ is a polynomial of degree $p(V, W)$. This polynomial is quite remarkable. It is closely related to polynomials introduced in [Dr2] for a description of finite dimensional representations of Yangians.

In some cases polynomials $Q^{V W}(z)$ can be computed explicitly [J1]. For example, if $V, W$ are fundamental $n$-dimensional representations of $U_{q}(\hat{\mathfrak{g}})$ for $\mathfrak{g}=\mathfrak{s l}(n)$, we have:

$$
\begin{aligned}
Q^{V W}(z)= & \left(q-z q^{-1}\right) \sum_{i=1}^{n} E_{i i} \otimes E_{i i} \\
& +(1-z) \sum_{i \neq j} E_{i i} \otimes E_{j j} \\
& +\left(q-q^{-1}\right) \sum_{i<j} E_{i j} \otimes E_{j i} \\
& +z\left(q-q^{-1}\right) \sum_{i>j} E_{i j} \otimes E_{j i}
\end{aligned}
$$

The function $r_{V W}(z)$ can be easily computed in this case as well:

$$
r_{V W}(z)=\frac{(1-z)\left(1-z q^{n}\right)}{\left(1-z q^{n-1}\right)\left(1-z q^{n+1}\right)} .
$$

As a simple corollary of relation (4.7), we obtain the Yang-Baxter relation for $R^{V W}(z)$ :

$$
R_{12}^{V W}(z) R_{13}^{V U}(z w) R_{23}^{W U}(w)=R_{23}^{W U}(w) R_{13}^{V U}(z w) R_{12}^{V W}(z)
$$

Now we are going to introduce "current type" generators for $U_{q}(\hat{\mathfrak{g}})$.

Let

$$
\begin{aligned}
& L^{+, V}(z)=\left(\mathrm{id} \otimes \pi_{V(1)}\right)\left(R_{21}\left(z q^{\frac{c}{2}}\right)\right) \\
& L^{-, V}(z)=\left(\operatorname{id} \otimes \pi_{V(1)}\right)\left(R\left(z^{-1} q^{-\frac{c}{2}}\right)\right)
\end{aligned}
$$


So, by definition $L^{ \pm, V}(z) \in \operatorname{End}(V) \otimes U_{q}(\hat{\mathfrak{g}}) \otimes \mathbf{C}\left[\left[z^{ \pm 1}\right]\right]$,

$$
\begin{aligned}
R^{V W}\left(\frac{z}{w}\right) L_{1}^{+, V}(z) L_{2}^{+, W}(w) & =L_{2}^{+, W}(w) L_{1}^{+, V}(z) R^{V W}\left(\frac{z}{w}\right) \\
R^{V W}\left(\frac{z}{w} q^{-c}\right) L_{1}^{+, V}(z) L_{2}^{-, W}(w) & =L_{2}^{-, W}(w) L_{1}^{+, V}(z) R^{V W}\left(\frac{z}{w} q^{+c}\right) \\
R^{V W}\left(\frac{z}{w}\right) L_{1}^{-, W}(z) L_{2}^{-, W}(w) & =L_{2}^{-, W}(w) L_{1}^{-, V}(z) R^{V W}\left(\frac{z}{w}\right) .
\end{aligned}
$$

Here $V$ and $W$ is a pair of two finite-dimensional representations.

From the formulas (4.9) for the action of the comultiplication on $R(z)$ we have:

$$
\begin{aligned}
& \Delta^{\prime}\left(L^{+, V}(z)\right)=L^{+, V}\left(z q^{-\frac{c_{2}}{2}}\right) \otimes L^{-, V}\left(z q^{\frac{c_{1}}{2}}\right), \\
& \Delta^{\prime}\left(L^{-, V}(z)\right)=L^{-, V}\left(z q^{\frac{c_{2}}{2}}\right) \otimes L^{-, V}\left(z q^{-\frac{c_{1}}{2}}\right),
\end{aligned}
$$

where $\otimes$ means the tensor product over $\operatorname{End}(V)$.

Fix some basis in $V$. Let $L^{ \pm, V}(z)=\sum_{n \geq 0} L_{(n)}^{ \pm, V} z^{ \pm, n}$. Then the explicit form (4.6) of the expansion of $R(z)$ implies the following:

Proposition 4.8. For any $V$, the matrix elements of $L_{(n)}^{ \pm, V}$ generates the algebra $U_{q}(\hat{\mathfrak{g}})$ (over $\mathbf{C}[[q-1]])$ and $L_{(n)}^{+, V}, L_{(n)}^{-, V}$ generate subalgebras $U_{q}\left(\hat{\mathfrak{b}}_{-}\right)$and $U_{q}\left(\hat{\mathfrak{b}}_{+}\right)$respectively.

Let $U_{q}\left(\hat{\mathfrak{g}}_{+}\right)$be a subalgebra of $U_{q}(\hat{\mathfrak{g}})$ generated by $e_{0}, e_{i}, f_{i}, i=1, \ldots, l$. It is a quantization of the universal enveloping algebra of the maximal parabolic Lie subalgebra $\hat{\mathfrak{g}}_{+} \hookrightarrow \hat{\mathfrak{g}}$. For $U_{q}(\mathfrak{g})$-module $V$ we define a $U_{q}\left(\hat{\mathfrak{g}}_{+}\right)$module structure on $V$ such that $e_{0} V=0, \pi_{V}(c)=k I_{V}$. Set $V_{k}$ to be $U_{q}(\hat{\mathfrak{g}})$-module induced from $U_{q}\left(\hat{\mathfrak{g}}_{+}\right)$-module $V$ :

$$
V_{k}=\operatorname{Ind}_{U_{q}\left(\hat{\mathfrak{g}}_{+}\right)}^{U_{q}(\hat{\mathrm{g}})} V .
$$

If the representation $V$ is irreducible with the highest weight $\lambda$ we will denote corresponding $U_{q}(\hat{\mathfrak{g}})$-module as $V_{\lambda, k}$.

As well as corresponding $\hat{\mathfrak{g}}$-modules, representations $V_{k}$ are naturally $\mathbf{Z}$-graded modules $V_{k}=\bigoplus_{n \geq 0} V_{k}[-n]$, where $V_{k}[0] \cong V$.

Let us describe the action of $L^{ \pm, V}(z)$ in highest weight modules.

Proposition 4.19. Let $\pi_{\lambda, k}: U_{q}(\hat{\mathfrak{g}}) \rightarrow \operatorname{End}\left(V_{\lambda, k}\right)$ be a highest weight representation of $U_{q}(\hat{\mathfrak{g}})$ then

$$
\left(\pi_{\lambda, k} \otimes \mathrm{id}\right) L^{ \pm, V}(z) \in \operatorname{End}\left(V_{\lambda, k}\right) \otimes \operatorname{End}(V) \otimes \mathbf{C}((z)) .
$$

This proposition follows from the definition of $L^{ \pm, V}(z)$ and from the fact that any vector of the finite level from $V_{k}^{\lambda}$ is annihilated by $L_{(n)}^{-, V}$ for sufficiently large $n$.

Let $V_{\lambda, k}^{*}$ be a $\mathbf{Z}$-graded $U_{q}(\hat{\mathfrak{g}})$-module dual to $V_{\lambda, k}$ :

$$
V_{\lambda, k}=\bigoplus_{n \geq 0}\left(V_{\lambda, k}[-n]\right)^{*}
$$

where $\left(V_{\lambda, k}[-n]\right)^{*}$ are finite dimensional spaces which are dual to $V_{\lambda, k}[-n]$. The action of $U_{q}(\hat{\mathfrak{g}})$ in $V_{\lambda, k}$ is usual:

$$
\left\langle a v^{\prime}, v\right\rangle=\left\langle v^{\prime}, S(a) v\right\rangle, \quad v^{\prime} \in V_{\lambda, k}^{*}, \quad v \in V_{\lambda, k} .
$$


The module $V_{\lambda, k}^{*}$ is a lowest weight module with the lowest weight vector $v_{\lambda}^{\prime}$ which is dual to highest weight vector $v_{\lambda} \in V_{\lambda, k}$. We have:

$$
k_{i} v_{\lambda}^{\prime}=q^{-\lambda_{i}} v_{\lambda}^{\prime}, \quad f_{i} v_{\lambda}^{\prime}=0, \quad c v_{\lambda}^{\prime}=k v_{\lambda}^{\prime} .
$$

Lemma 4.3. (1) Elements $L^{ \pm, V}(z)$ have the following action on zero-level subspaces of $V_{\lambda, k}$ and $V_{\lambda, k}^{*}$ respectively:

$$
\begin{aligned}
L^{+, V}(z)\left(u^{\prime} \otimes v\right) & =\bar{R}^{V_{\lambda, k}[0], V}\left(u^{\prime} \otimes v\right), \\
L^{-, V}(z)(u \otimes v) & =\left(\bar{R}^{V, V_{\lambda, k}[0]}\right)^{-1}(u \otimes v) .
\end{aligned}
$$

Here $u \in V_{\lambda, k}[0], u^{\prime} \in V_{\lambda, k}^{*}[0], v \in V$ and $\bar{R}^{V, W}$ is a $U_{q}(\mathfrak{g})-R$-matrix acting in $V \otimes W$.

(2) If $u=v_{\lambda}$ or $u^{\prime}=v_{\lambda}^{\prime}$, we have

$$
\begin{aligned}
& L^{+, V}(z)\left(v_{\lambda}^{\prime} \otimes v\right)=q^{\lambda} v_{\lambda}^{\prime} \otimes v, \\
& L^{-, V}(z)\left(v_{\lambda} \otimes v\right)=v_{\lambda} \otimes q^{-\lambda} v .
\end{aligned}
$$

Proof. As it follows from the definition of $L^{+, V}(z)$, it has the form $L^{+, V}(z)=$ $\sum_{n \geq 0} \sum_{i} \pi_{V}\left(b^{i}(n)\right) \otimes a_{i}(n) z^{n}$, where $a^{i}(n) \in U_{q}\left(\hat{\mathfrak{b}}_{+}\right), b^{i}(n) \in U_{q}\left(\hat{\mathfrak{b}}_{-}\right)$. Any monomial from $U_{q}\left(\hat{\mathfrak{b}}_{+}\right)$which contains $e_{0}$, annihilates any vector of level zero. Therefore in (4.41), only those monomials $a^{i}(n)$ will act nontrivially which contain only real root generators. As we have seen in Sect. 3, these monomials form $\bar{R}^{V, W}$. The proof of other statements is similar.

Now we would like to exhibit the relation between the generating functions $L^{ \pm, V}(z)$ and their undeformed analogues $J^{ \pm}(z)$. Since the algebra $U_{q}(\hat{\mathfrak{g}})$ is a deformation of $U(\hat{\mathfrak{g}})$ in the sense of the identification $U(\hat{\mathfrak{g}}) \cong U_{q}(\hat{\mathfrak{g}}) /(q-1) U_{q}(\hat{\mathfrak{g}})$ we can identify the first nontrivial term of the $(q-1)$-expansion of $L^{ \pm, V}(z)$ with an element in $U(\hat{\mathfrak{g}})$. One can show that

$$
L^{ \pm, V}(z)=1 \otimes I_{V}+(q-1) \sum_{a=1}^{\operatorname{dim} \mathfrak{g}} \tilde{J}_{a}^{ \pm}(z) \otimes \pi_{V}\left(J_{a}\right)+O\left((q-1)^{2}\right),
$$

where $a$ is a basis of $\mathfrak{g}, \tilde{J}_{a}^{ \pm}(z)$ are the generating functions (2.24) for $\hat{\mathfrak{g}}$, in the Borel polarization, whose components satisfy the commutation relations (2.1) of the affine Lie algebra.

For any highest weight module $\hat{V}$ with $c=k$, define the operator

$$
\begin{aligned}
& \left(\pi_{\hat{V}} \otimes \mathrm{id}\right)\left(L^{V}(z)\right) \stackrel{\text { def }}{=}\left(\pi_{\hat{V}} \otimes \mathrm{id}\right)\left(L^{+, V}\left(z q^{-\frac{k}{2}}\right)\right) \\
& \quad \times\left(\pi_{\hat{V}} \otimes \mathrm{id}\right)\left(S\left(L^{-, V}\left(z q^{\frac{k}{2}}\right)\right) \in \operatorname{End}(\hat{V}) \otimes \operatorname{End}(V) \otimes \mathbf{C}((z)) .\right.
\end{aligned}
$$

Assuming that it acts only on highest weight modules, we will denote this operator just as $L^{V}(z)$.

From (4.33) we have the following relations for $L^{V}(z)$ :

$$
\begin{aligned}
& R_{12}\left(\frac{z}{w}\right) L_{1}(z) R_{12}^{-1}\left(\frac{z}{w} q^{2 k}\right) L_{2}(w) \\
& \quad=L_{2}(w) R_{12}\left(\frac{z}{w} q^{-2 k}\right) L_{2}(w) R_{12}^{-1}\left(\frac{z}{w}\right) .
\end{aligned}
$$


This operator is a quantum analogue of "current" generator:

$$
L^{V}(z)=1 \otimes I_{V}-(q-1) \sum_{\alpha} J_{a}(z) \otimes \pi_{V}\left(J_{a}\right)+O\left((q-1)^{2}\right),
$$

where $J_{a}$ is a basis in $\mathfrak{g}, J_{a}(z)$ are "currents" for $\hat{\mathfrak{g}}$ (see Sect. 2).

Remark 4.2. In the case of affine Lie algebra $\hat{\mathfrak{g}}$ any finite-dimensional representations can be obtained from the tensor product of the evaluation representation $V_{\lambda}(z)$ described in Sect.2. In this case the parameter $z$ is fixed by the conditions $\pi_{\lambda}\left(e_{0}\right)=z \pi_{\lambda}\left(x_{-\theta}\right)$ and $\pi_{\lambda}\left(f_{0}\right)=z^{-1} \pi_{\lambda}\left(x_{\theta}\right)$, where $x_{\theta}$ and $x_{-\theta}$ are elements of the Chevalley basis of $\mathfrak{g}$, corresponding to the maximal root $\theta$ and its negative. In the $q$-deformed case of $U_{q}(\hat{\mathfrak{g}})$ there exist analogues of the evaluation representations. In particular, one can always extend an irreducible representation $V_{\lambda}$ of $U_{q}(\mathfrak{s l}(n))$ to a representation $V_{\lambda}(z)$ of $U_{q}(\widehat{\mathfrak{s l}}(n))$. Then the parameter $z$ can be chosen consistently with the undeformed case. For other types of quantum algebras $U_{q}(\mathfrak{g})$ one generally has to enlarge $V_{\lambda}$ by adding certain "smaller" irreducible representations in order to extend the resulting representation to $U_{q}(\hat{\mathfrak{g}})$. An explicit description of this extension is an important open problem.

We conclude the section introducing intertwining operators which are analogues of (2.8). For highest weight $U_{q}(\hat{\mathfrak{g}})$ modules $V_{\lambda, k}$ and $V_{\mu, k}$ with $c=k$ and for finite dimensional $V$ we define intertwining operators

$$
\left(\Phi^{V}(z)\right)_{\lambda}^{\mu}: V_{\lambda, k} \rightarrow V_{\mu, k} \otimes V(z) \otimes \mathbf{C}((z)) \cdot z^{h(\lambda)-h(\mu)}
$$

where $h(\lambda)$ and $h(\mu)$ are the same as in Sect. 3 .

From the definition of these intertwiners and from (4.34), we have the following relations (we will omit indices $\lambda$ and $\mu$ of $\Phi^{V}(z)$ when it is not misleading

$$
\begin{aligned}
& \Phi_{1}^{V}(z) L_{2}^{+, W}(w)=R_{21}^{W V}\left(\frac{w}{z} q^{-\frac{k}{2}}\right) L_{2}^{+, W}(w) \Phi_{1}^{V}(z) \\
& \Phi_{1}^{V}(z) L_{2}^{-, W}(w)=R_{21}^{W V}\left(\frac{w}{z} q^{\frac{k}{2}}\right) L_{2}^{-, W}(w) \Phi_{1}^{V}(z)
\end{aligned}
$$

In the next section, we derive difference equation for intertwining operators (4.46), which is an analogue of Eq. (2.29).

\section{Difference Equations for Intertwining Operators}

In this section with all definitions in hand we are ready to derive the quantum analogue of the Knizhnik-Zamolodchikov equations following the similar line of arguments as in Sect. 2.

Theorem 5.1. The intertwining operator $\Phi^{V}(z)$ satisfies the following difference equation:

$$
\Phi^{V}\left(z q^{-(k+g)}\right)=\vdots L^{V}\left(z q^{-g}\right) \Phi^{V}\left(z q^{k+g}\right)
$$

where

$$
\vdots L^{V}(z) \Phi^{V}(z) \vdots=L^{+}\left(z q^{-\frac{k}{2}}\right)\left(\Phi^{V}(z)^{t}\left(q^{2 \varrho}\right)^{t} S\left(L^{-}\left(z q^{\frac{k}{2}}\right)\right)^{t}\right)^{t}
$$


Proof. The proof is similar to the proof of Theorem 2.1 and uses an induction by the level of vectors in the matrix coefficients of both sides of (5.1). We have to prove that for each $v \in V_{\lambda, k}$ and for each $v^{\prime} \in V_{\mu, k}^{*}$ we will have:

$$
\left\langle v^{\prime}, \Phi^{V}\left(z q^{-(k+g)}\right) v\right\rangle=\left\langle v^{\prime}, \vdots L^{V}\left(z q^{-g}\right) \Phi^{V}\left(z q^{(k+g)}\right) \vdots v\right\rangle .
$$

Assume that $v^{\prime} \in V_{\mu, k}^{*}[-n], v \in V_{\lambda, k}[-m]$ are elements of corresponding graded components of $V_{\mu, k}^{*}$ and $V_{\lambda, k}$, respectively.

Lemma 5.1. Left- and right-hand sides of (5.1) have the same relations (4.48) with elements $L^{\mp, V}(z)$. They are both intertwining operators of type (4.47).

Proof. Direct computations and Proposition 4.9.

We will prove (5.3) by induction over $n+m$ using this lemma.

1. Proof of the induction step. Assume that we proved (5.3) for any $(n, m)$ such that $n+m \leq N$. Any $v \in V_{\lambda, k}[-(m+1)]$ can be presented as a linear combination of the vectors $L_{i j(-r)}^{+, V} u$ for some $u \in \bigoplus_{s \leq m} V_{\lambda, k}[-s]$ and $r>0$. By Lemma 5.1, the commutation relations of $L_{i j(r)}^{+, V}$ with both sides of (5.1) are the same. Besides the result of the commutation belongs to the level $(n-r, m-r)$. The similar argument can be applied to any $v^{\prime} \in V_{\lambda, k}^{*}[n+1]$, which can be presented as a linear combination of the vectors $L_{i j(r)}^{-, V} u^{\prime}$ for some $u^{\prime} \in \bigoplus_{s \leq n} V_{\mu, k}^{*}[s]$ and $r>0$.

2. Proof of the induction base. Let $n=m=0$. From Lemma 4.4 we conclude that we have to prove in this case the following equality:

$$
\left\langle v^{\prime}, \Phi^{V}\left(q^{-(k+g)} z\right) v\right\rangle=\left\langle v^{\prime}, \bar{R}^{V_{\mu}, V}\left(\Phi^{V}(z)^{t}\left(q^{-\varrho}\right)_{V}^{t}\left(\bar{R}_{21}^{V, V_{\lambda}}\right)^{t_{2}}\right)^{t} v\right\rangle .
$$

We have used here isomorphisms:

$$
V_{\mu, k}[0]^{*} \cong V_{\mu}^{*}, \quad V_{\lambda, k}[0] \cong V_{\lambda} .
$$

Now, using Theorem 3.2, we can transform this equation to

$$
\left\langle v^{\prime}, \Phi^{V}\left(z q^{-2(k+g)}\right) v\right\rangle=q^{C(\lambda)-C(\mu)}\left\langle v^{\prime}, \Phi^{V}(z) v\right\rangle .
$$

From the definition of $\Phi^{V}(z)$ we have

$$
\left\langle v^{\prime}, \Phi^{V}(z) v\right\rangle=z^{h(\mu)-h(\lambda)}\left\langle v^{\prime}, \Phi^{V}[0] v\right\rangle .
$$

And therefore Eq. (5.3) holds.

The relation (4.46) shows that (5.1) is indeed a $q$-analogue of (2.29) in the sense that it gives the last one when $q \rightarrow 1$.

Denote

$$
H_{\mu, \lambda}^{V}=\operatorname{Hom}_{U_{g}(g)}\left(V_{\lambda}, V_{\mu} \otimes V\right) .
$$

Corollary 5.1. The dimension of the space of intertwining operators $\left(\Phi^{V}(z)\right)_{\lambda}^{\mu}$ is equal to $\operatorname{dim}\left(H_{\lambda \mu}^{V}\right)$.

Proof. The proof of the previous theorem was constructive and, in fact, we proved that if $\left\langle v^{\prime}, \Phi^{V}[0] v\right\rangle$ is nonzero then $\Phi^{V}(z)$ is also nonzero. But $\Phi^{V}$ [0] acts on zero level as $U_{q}(\mathfrak{g})$ intertwiner from $H_{\mu \lambda}^{V}$.

Let $a \in H_{\mu \lambda}^{V}$, then the corresponding intertwining operator $\left(\Phi^{V}(z)\right)_{\lambda}^{\mu}$ will be also denoted as $\left(\Phi^{V}(z \mid a)\right)_{\lambda}^{\mu}$. 
Now we consider a product of intertwining operators

$$
\begin{aligned}
& \Phi_{\lambda_{0} \ldots \lambda_{N}}^{V_{1} \ldots V_{N}}\left(z_{1}, \ldots, z_{N}\right): V_{\lambda_{\nu, k}} \rightarrow V_{\lambda_{0}, k} \otimes V_{1} \otimes \cdots \otimes V_{N} \\
& \quad \otimes \prod_{i=1}^{N} z_{i}^{h\left(\lambda_{i-1}\right)-h\left(\lambda_{i}\right)} \mathbf{C}\left(\left(z_{1}, \frac{z_{2}}{z_{1}}, \frac{z_{3}}{z_{2}}, \ldots, \frac{z_{N}}{z_{N-1}}\right)\right) \Phi_{\lambda_{0} \ldots \lambda_{N}}^{V_{1} \ldots V_{N}}\left(z_{1}, \ldots, z_{N}\right) \\
& \quad=\left(\left(\Phi^{V_{1}}\left(z_{1}\right)\right)_{\lambda_{1}}^{\lambda_{0}} \otimes I_{V_{2} \otimes \cdots \otimes V_{N}}\right) \cdot\left(\left(\Phi^{V_{2}}\left(z_{2}\right)\right)_{\lambda_{2}}^{\lambda_{1}} \otimes I_{V_{3} \otimes \cdots \otimes V_{N}}\right) \ldots\left(\Phi^{\lambda_{N}}\left(z_{N}\right)\right)_{\lambda_{N}}^{\lambda_{N-1}} .
\end{aligned}
$$

We will also use the abbreviation as in Sect. 2:

$$
\Phi_{\lambda_{0} \ldots \lambda_{N}}^{V_{1} \ldots V_{N}}\left(z_{1}, \ldots, z_{N}\right)=\left(\Phi_{1}^{V_{1}}\left(z_{1}\right)\right)_{\lambda_{1}}^{\lambda_{0}}\left(\Phi_{2}^{V_{2}}\left(z_{2}\right)\right)_{\lambda_{2}}^{\lambda_{1}} \ldots\left(\Phi_{N}^{V_{N}}\left(z_{N}\right)\right)_{\lambda_{N}}^{\lambda_{N-1}} .
$$

We will omit the subindices $\lambda_{0}, \ldots, \lambda_{N}$ when it will not lead to misunderstanding.

Let

$$
T_{j}\left(\Phi^{V_{1} \ldots V_{N}}\left(z_{1}, \ldots, z_{N}\right)\right)=\Phi^{V_{1} \ldots V_{N}}\left(z_{1}, \ldots, z_{j} q^{-2(k+g)}, \ldots, z_{N}\right)
$$

and denote $p=q^{-2(k+g)}$.

Theorem 5.2. The product of intertwining operators (5.4) satisfies the following system of difference equations:

$$
\begin{aligned}
T_{j}\left(\Phi^{V_{1} \ldots V_{N}}\left(z_{1}, \ldots, z_{N}\right)\right) \\
=R_{j j-1}^{V_{j} V_{j-1}}\left(\frac{z_{j}}{z_{j-1}} p\right) \ldots R_{j 1}^{V_{j} V_{1}}\left(\frac{z_{j}}{z_{1}} p\right) L_{j}^{+, V_{j}}\left(z_{j} q^{-\frac{3 k}{2}-2 g}\right) \Phi^{V_{1} \ldots V_{j-1}}\left(z_{1}, \ldots, z_{j-1}\right) \\
\quad \times\left(\Phi^{V_{j}}\left(z_{j}\right)^{t_{j}} R_{j j-1}\left(\frac{z_{j}}{z_{j-1}}\right)^{t_{j}} \ldots R_{j 1}\left(\frac{z_{j}}{z_{1}}\right)^{t_{j}}\left(q^{2 \varrho}\right)_{j}^{t_{j}} S\left(L_{j}^{-, V_{j}}\left(z_{j} q^{-\frac{k}{2}-2 g}\right)\right)^{t_{j}}\right)^{t_{j}} \\
\quad \times \Phi^{V_{j+1} \ldots V_{N}}\left(z_{j+1}, \ldots, z_{N}\right) .
\end{aligned}
$$

Proof. Relations (5.1) and (4.48) imply:

$$
\begin{aligned}
T_{j} & \left(\Phi^{V_{1} \ldots V_{N}}\left(z_{1}, \ldots, z_{N}\right)\right) \\
= & R_{j j-1}^{V_{j} V_{j-1}}\left(\frac{z_{j}}{z_{j-1}} p\right) \ldots R_{j 1}^{V_{j} V_{1}}\left(\frac{z_{j}}{z_{1}} p\right) L_{j}^{+, V_{\jmath}}\left(z_{j} q^{-\frac{3 k}{2}-2 g}\right) \Phi^{V_{1} \ldots V_{j-1}}\left(z_{1}, \ldots, z_{j-1}\right) \\
& \times\left(\Phi^{V_{j}}\left(z_{j}\right)^{t_{j}}\left(q^{2 \varrho}\right)_{j}^{t_{\jmath}} S\left(L_{j}^{-, V_{j}}\left(z_{j} q^{-\frac{k}{2}-2 g}\right)^{t_{\jmath}}\right)^{t_{j}} \Phi^{V_{j+1} \ldots V_{N}}\left(z_{j+1}, \ldots, z_{N}\right) .\right.
\end{aligned}
$$

From (4.48) we can also deduce the following relation:

$$
S\left(L_{2}^{-, V}(z)\right)^{t_{2}} \Phi_{1}^{V_{1}}(w)=\left(R_{12}\left(\frac{w}{z} q^{-\frac{k}{2}}\right)^{t_{2}}\right)^{-1} \cdot \Phi_{1}^{V_{1}}(w) \cdot S\left(L_{2}^{-, V}(z)\right)^{t_{2}}
$$

Using repeatedly this relation, we obtain (5.7).

Now we consider matrix coefficients of products of the intertwining operators

$$
\begin{gathered}
\mathscr{F}_{\lambda_{0} \ldots \lambda_{N}}^{V_{1} \ldots V_{N}}\left(z_{1}, \ldots, z_{N}\right) \in V_{\lambda_{0}} \otimes V_{N} \otimes \cdots \otimes V_{1} \otimes\left(V_{\lambda_{N}}\right)^{*} \\
\otimes \prod_{i=1}^{N} z_{i}^{h\left(\lambda_{i-1}\right)-h\left(\lambda_{i}\right)} \mathbf{C}\left(\left(z_{i}, \frac{z_{2}}{z_{1}}, \ldots, \frac{z_{N}}{z_{N-1}}\right)\right), \\
\mathscr{F}_{\lambda_{0} \ldots \lambda_{N}}^{V_{1} \ldots V_{N}}\left(z_{1}, \ldots, z_{N}\right)=\sum_{i}\left\langle\cdot, \Phi_{\lambda_{0} \ldots \lambda_{N}}^{V_{1} \ldots V_{N}}\left(z_{1}, \ldots, z_{N}\right) \cdot\right\rangle .
\end{gathered}
$$


Theorem 5.3. The matrix coefficients defined by (5.8), (5.9) satisfy the following system of difference equations:

$$
\begin{aligned}
T_{j}\left(\mathscr{F}_{\lambda_{0} \ldots \lambda_{N}}^{V_{1} \ldots V_{N}}\left(z_{1}, \ldots, z_{N}\right)\right) \\
=R_{j j-1}^{V_{j} V_{j-1}}\left(\frac{z_{j}}{z_{j-1}} p\right) \ldots R_{j 1}^{V_{j} V_{1}}\left(\frac{z_{j}}{z_{1}} p\right) \bar{R}_{j, 0}^{V_{j}, V_{\lambda_{0}}}\left(q^{2 \varrho}\right)_{j} \cdot\left(\bar{R}_{N+1, j}^{V_{\lambda_{N}, V_{j}}^{*}}\right)^{-1} \\
\quad \times R_{j N}^{V_{j} V_{N}}\left(\frac{z_{j}}{z_{N}}\right) \ldots R_{j j-1}^{V_{j} V_{j-1}}\left(\frac{z_{j}}{z_{j-1}}\right) \mathscr{F}_{\lambda_{0} \ldots \lambda_{N}}^{V_{1} \ldots V_{N}}\left(z_{1}, \ldots, z_{N}\right) \\
\equiv A_{j}\left(z_{1}, \ldots, z_{N}\right) \cdot \mathscr{F}_{\lambda_{0} \ldots \lambda_{N}}^{V_{1} \ldots V_{N}}\left(z_{1}, \ldots, z_{N}\right) .
\end{aligned}
$$

Here $\bar{R}^{V, W}$ is the $U_{q}(\mathfrak{g})-R$-matrix, acting in $V \otimes W ; \bar{R}^{V W}=R^{V W}(0)$.

Proof. The theorem follows immediately from Theorem 5.2 and from the action of $L^{\mp, V}(x)$ on zero level subspace in lowest weight and highest weight modules, respectively. The explicit expression for this action is given by Lemma 4.4 .

Thus we obtained a new system of difference equations together with its solutions given in the form of power series. In fact, the matrix coefficients (5.8), (5.9) form a certain subspace in the space of all solutions of the system (5.10). The description of this subspace is given by the following proposition.

\section{Proposition 5.1.}

$$
\begin{aligned}
& \mathscr{F}_{\lambda_{0} \ldots \lambda_{N}}^{V_{1} \ldots V_{N}}\left(z_{1}, \ldots, z_{N}\right) \in \operatorname{Inv}_{U_{q}(\mathfrak{g})}\left(V_{\lambda_{0}} \otimes V_{1} \otimes \cdots \otimes V_{N} \otimes\left(V_{\lambda_{N}}\right)^{*}\right) \\
& \otimes\left(\prod_{i=1}^{N} z_{i}^{k\left(\lambda_{i-1}\right)-k\left(\lambda_{i}\right)} \mathbf{C}\left(\left(z_{1}, \frac{z_{2}}{z_{1}}, \ldots, \frac{z_{N}}{z_{N-1}}\right)\right)\right) .
\end{aligned}
$$

(2) The dimension of the space of intertwining operators of type $\Phi_{\lambda_{0} \ldots \lambda_{N}}^{V_{1} \ldots V_{N}}\left(z_{1}, \ldots, z_{N}\right)$, where $\lambda_{i}, i=1, \ldots, N$, run through all the dominant weights, is equal to

$$
\operatorname{dim}\left(\operatorname{Inv}_{U_{q}(\mathfrak{g})}\left(V_{\lambda_{0}} \otimes V_{1} \otimes \cdots \otimes V_{N} \otimes\left(V_{\lambda_{N}}\right)^{*}\right) .\right.
$$

(3) $\operatorname{Inv}_{U_{q}(\mathfrak{g})}\left(V_{\lambda_{0}} \otimes V_{1} \otimes \cdots \otimes V_{N} \otimes\left(V_{\lambda_{N}}\right)^{*}\right)$ is an invariant subspace for linear operators $A_{j}\left(z_{1}, \ldots, z_{N}\right)$.

Proof. (1) This statement follows immediately from the form of $\mathscr{F}$ as matrix coefficients of a product of the intertwining operators, which by definition commute with the action of $U_{q}(\mathfrak{g})$.

(2) Corollary 5.1 implies that an intertwining operator is uniquely determined by its zero level component. The space of zero level components for the product $\Phi_{\lambda_{0} \ldots \lambda_{N}}^{V_{1} \ldots V_{N}}$ for all possible $\lambda_{i}, i=1, \ldots, N$, has the dimension equal to

$$
\operatorname{dim} H_{\lambda_{0}, \lambda_{N}}^{V_{1} \otimes \cdots V_{N}}=\operatorname{dim}\left(\operatorname{Inv}_{U_{q}(\mathfrak{g})}\left(V_{\lambda_{0}} \otimes V_{1} \otimes \cdots \otimes V_{N} \otimes\left(V_{\lambda_{N}}^{*}\right)\right) .\right.
$$

(3) It follows from the commutativity $A_{j}\left(z_{1}, \ldots, z_{N}\right)$ with the action of $U_{q}(\mathfrak{g})$. The latter is a corollary of the formula for the comultiplication [see (3.16)].

$$
\begin{gathered}
\left(\pi_{\lambda_{0}} \otimes \pi_{1} \otimes \cdots \otimes \pi_{N} \otimes \pi_{\lambda_{N}}^{*}\right)\left(\Delta^{N+1}\left(L^{+, V}\right)\right) \\
=R^{V V_{\lambda_{0}} R^{V V_{1}} \ldots R^{V V_{N}}\left(R^{V_{\lambda_{N}}^{*} V}\right)^{-1}}
\end{gathered}
$$

(similar for $L^{-, V}$ ) and the Yang-Baxter identity (4.31) with $z=0$. 
We will see in the next section that the system (5.10) gives an alternative characterization of matrix coefficients of products of intertwining operators, as $U_{q}(\mathfrak{g})$-invariant solutions of (5.10) meromorphic for all values $z_{i} \neq 0, \infty$. As a corollary, we will obtain an "exchange algebra" for the intertwining operators.

The system (5.10) simplifies if we define a vector function

$$
\begin{aligned}
\Psi_{\lambda_{0} \ldots \lambda_{N}}^{V_{1} \ldots V_{N}}\left(z_{1}, \ldots, z_{N}\right) \in V_{1} \otimes \cdots \otimes V_{N} \\
\quad \otimes\left(\prod_{i=1}^{N} z_{i}^{h\left(\lambda_{i-1}\right)-h\left(\lambda_{i}\right)} \mathbf{C}\left(\left(z_{1}, \frac{z_{2}}{z_{1}}, \ldots, \frac{z_{N}}{z_{N-1}}\right)\right)\right)
\end{aligned}
$$

as

$$
\Psi_{\lambda_{0} \ldots \lambda_{N}}^{V_{1} \ldots V_{N}}\left(z_{1}, \ldots, z_{N}\right)=\left\langle v_{\lambda_{0}}^{\prime}, \Phi_{\lambda_{0} \ldots \lambda_{N}}^{V_{1} \ldots V_{N}}\left(z_{1}, \ldots, z_{N}\right) v_{\lambda_{N}}\right\rangle
$$

where $v_{\lambda_{0}}^{\prime}$ is a lowest weight vector in $V_{\lambda_{0}, k}^{*}$ and $v_{\lambda_{N}}$ is a highest weight vector in $V_{\lambda_{N}, k}$.

It follows from Theorem 5.3 that

$$
\begin{aligned}
T_{j}( & \left.\Psi_{\lambda_{0} \ldots \lambda_{N}}^{V_{1} \ldots V_{N}}\left(z_{1}, \ldots, z_{N}\right)\right) \\
= & R_{j j-1}^{V_{i} V_{j-1}}\left(\frac{z_{j}}{z_{j-1}} p\right) \ldots R_{j 1}^{V_{j} V_{1}}\left(\frac{z_{j}}{z_{1}} p\right)\left(q^{\lambda_{0}+\lambda_{N}+2 \varrho}\right)_{j} R_{j N}^{V_{j} V_{N}}\left(\frac{z_{j}}{z_{N}}\right) \\
& \ldots R_{j j+1}^{V_{j} V_{j+1}}\left(\frac{z_{j}}{z_{j+1}}\right) \Psi_{\lambda_{0} \ldots \lambda_{N}}^{V_{1} \ldots V_{N}}\left(z_{1}, \ldots, z_{N}\right) .
\end{aligned}
$$

We will use this system in Sect. 7 in order to compute explicitly solutions of (5.10) for $U_{q}\left(\widehat{\mathfrak{s l}_{2}}\right)$.

Thus we found a collection of systems of commuting difference equations from the analysis of the representation theory of $U_{q}(\hat{\mathfrak{g}})$. The main ingredients in these systems are solutions of the Yang-Baxter equation. Systems of this type are interesting by itself. They are examples of $q$-holonomic systems studied by Aomoto and others [A1, A2, A3, M]. Now we can construct generalizations of systems (5.11) to a more general class of solutions to the Yang-Baxter equation.

Let us assume the following:

$1^{0}$ ) For a collection of vector spaces $V_{1}, \ldots, V_{N}$ and a set $\Gamma \subset \mathbf{C}$ of complex codimension 1 , we have a set of linear maps $R^{V_{\imath} V_{\jmath}}(x)$, where $x \in \mathbf{C}$ and $R^{V W}(x)$ are nondegenerate for $x \in \mathbf{C} \backslash \Gamma$.

$2^{0}$ ) The maps $R^{V_{\imath} V_{J}}(x)$ satisfy the Yang-Baxter relation:

$$
R_{12}^{V_{1} V_{2}}(x) R_{13}^{V_{1} V_{3}}(x+y) R_{23}^{V_{2} V_{3}}(y)=R_{23}^{V_{2} V_{3}}(y) R_{13}^{V_{1} V_{3}}(x+y) R_{12}^{V_{1} V_{2}}(x) .
$$

$3^{0}$ ) For each $i=1, \ldots, N$ we choose $d_{i} \in \operatorname{End}\left(V_{i}\right)$ such that

$$
\left(d_{i} \otimes d_{j}\right) R^{V_{i} V_{j}}(x)=R^{V_{\imath} V_{\jmath}}(x)\left(d_{i} \otimes d_{j}\right) .
$$

Then we have a theorem

Theorem 5.4. Let $\kappa \in \mathbf{C}, x_{i}-x_{j} \in \mathbf{C} \backslash \Gamma$ and

$$
\begin{aligned}
& A_{i}\left(x_{1}, \ldots, x_{N}\right) \\
& \quad=R_{i i-1}\left(x_{i}-x_{i-1}+\kappa\right) R_{i i-2}^{V_{2} V_{i-2}}\left(x_{i}-x_{i-2}+\kappa\right) \ldots R_{i 1}^{V_{i} V_{1}}\left(x_{i}-x_{1}+\kappa\right) d_{i} \\
& \quad \times R_{N i}^{V_{N} V_{\imath}}\left(x_{N}-x_{i}\right)^{-1} \ldots R_{i+1 i}^{V_{2+1} V_{i}}\left(x_{i+1}-x_{i}\right)^{-1} .
\end{aligned}
$$


Here we use the notation for $R$-matrices acting in $V_{1} \otimes \cdots \otimes V_{N}$ as in the previous sections. Then the system of difference equations

$$
\mathscr{F}\left(x_{1}, \ldots, x_{i}+\kappa, \ldots, x_{N}\right)=A_{i}\left(x_{1}, \ldots, x_{N}\right) \mathscr{F}\left(x_{1}, \ldots, x_{i}, \ldots, x_{N}\right)
$$

is a system of commuting difference equations.

Proof. The proof follows directly from the Yang-Baxter equation (5.14). We have to check that

$$
\left(T_{j} A_{i}\right)(x) A_{j}(x)=\left(T_{i} A_{j}\right)(x) A_{i}(x),
$$

where $T_{i} f\left(x_{1}, \ldots, x_{N}\right)=f\left(x_{1}, \ldots, x_{i}+\kappa, \ldots, x_{N}\right)$ and $A_{i}(x)$ are given by (5.16). In terms of $R$-matrices, this is the following identity (we assume $i<j$ )

$$
\begin{aligned}
R_{i i-1} & \left(x_{i}-x_{i-1}+\kappa\right) \ldots R_{i 1}\left(x_{i}-x_{1}+\kappa\right) d_{i} R_{N i}\left(x_{N}-x_{i}\right) \ldots R_{j i}\left(x_{j}-x_{i}+\kappa\right)^{-1} \\
& \ldots R_{i+1 i}\left(x_{i+1}-v_{x}\right)^{-1} \cdot R_{j j-1}\left(x_{j}-x_{j-1}+\kappa\right) \ldots R_{j i}\left(x_{j}-x_{i}+\kappa\right) \\
& \ldots R_{j 1}\left(x_{j}-x_{1}+\kappa\right) d_{\jmath} R_{N j}\left(x_{N}-x_{j}\right)^{-1} \ldots R_{j+1 j}\left(x_{j+1}-x_{j}\right)^{-1} \\
= & R_{\jmath \jmath-1}\left(x_{j}-x_{j-1}+\kappa\right) \ldots R_{j i}\left(x_{j}-x_{i}\right) \ldots R_{j 1}\left(x_{j}-x_{1}+\kappa\right) \\
& \times d_{j} R_{N j}\left(x_{N}-x_{j}\right)^{-1} \ldots R_{j+1 j}\left(x_{j+1}-x_{j}\right)^{-1} R_{i \imath-1}\left(x_{i}-x_{i-1}+\kappa\right) \\
& \ldots R_{i 1}\left(x_{i}-x_{1}+\kappa\right) d_{i} R_{N i}\left(x_{N}-x_{i}\right)^{-1} \ldots R_{i+1 i}^{-1}\left(x_{i+1}-x_{i}\right) .
\end{aligned}
$$

Here we omit the indices $V_{i}, V_{j}$ in $R_{i j}^{V_{i} V_{j}}\left(x_{i}-x_{j}\right)$. We first move $R_{i+1 i}\left(x_{i+1}-x_{i}\right)^{-1}$ in the left-hand side to the right and at the same time $R_{j i+1}\left(x_{j}-x_{i+1}+\kappa\right)$ to the left using

$$
\begin{aligned}
& R_{i+1 i}\left(x_{\imath+1}-x_{i}\right)^{-1} R_{j i+1}\left(x_{j}-x_{i+1}\right) R_{j i}\left(x_{j}-x_{\imath}+\kappa\right) \\
& \quad=R_{j i}\left(x_{j}-x_{i}+\kappa\right) R_{j i+1}\left(x_{j}-x_{i+1}+\kappa\right) R_{i+1, i}^{-1}\left(x_{i+1}-x_{i}\right),
\end{aligned}
$$

which follows from the Yang-Baxter relation. Then we repeat the same with $R_{i+2 i}\left(x_{i+2}-x_{i}\right)^{-1}$ until we reach $R_{j i}\left(x_{j}-x_{i}+\kappa\right)^{-1}$ which cancels $R_{j i}\left(x_{j}-x_{i}+\kappa\right)$. Then we consider the right-hand side and move $R_{j \imath-1}\left(x_{j}-x_{i-1}+\kappa\right)$ to the right and simultaneously $R_{i-1}\left(x_{i}-x_{i-1}+\kappa\right)$ to the left using

$$
\begin{aligned}
& R_{j i}\left(x_{j}-x_{i}\right) R_{j i-1}\left(x_{j}-x_{i-1}+\kappa\right) R_{i i-1}\left(x_{i}-x_{i-1}+\kappa\right) \\
& \quad=R_{\imath i-1}\left(x_{i}-x_{i-1}+\kappa\right) R_{j-1}\left(x_{j}-x_{i-1}+\kappa\right) R_{j i}\left(x_{j}-x_{i}\right),
\end{aligned}
$$

and so on until we cancel $R_{j i}\left(x_{j}-x_{i}\right)$ and $R_{j i}\left(x_{j}-x_{i}\right)^{-1}$. Once we use the identity (5.15).

Remark 5.1. In light of this theorem, we can regard the Yang-Baxter equation as a "flatness condition" for a "difference connection" (5.16), (5.17). Of course, in order to make sense of this sentence, we have to define what is the "difference connection."

There are three known basic types of $R$-matrices:

(i) with rational dependence of additive parameter $x$,

(ii) with trigonometric dependence of this parameter,

(iii) with elliptic dependence of the parameter $x$.

The representation theory of quasitriangular Hopf algebras $U_{q}(\hat{\mathfrak{g}})$ gives us trigonometric solutions of the Yang-Baxter equation and we studied them above. As it was pointed out earlier, trigonometric solutions $R^{V W}(z)$ related to $U_{q}(\hat{\mathfrak{g}})$ in the limit when $z=q^{x}$, and $q \rightarrow 1$ yield solutions of the Yang-Baxter equation with rational dependence of $x$. These solutions arise from representation theory of Yangians. 
The algebraic structure of the elliptic solution of the Yang-Baxter equation [B1, $\mathrm{Be} 1, \mathrm{JKMO}, \mathrm{JMO}$ ] has not been studied enough yet. Some results in this direction can be found in [Ch1]. For elliptic $R(x)$ the system (5.16) is a holonomic system on a torus and the rationality of $\kappa$ (with respect to module of the torus) will be important in this case.

In all three cases, it is natural to look for solutions of the difference equations with certain analytic properties, e.g. meromorphic in $\mathbf{C}^{N}$. In the next section we will show that the matrix coefficients of products of intertwining operators represented above by formal power series do have the right analytic properties.

\section{General Analysis of Solutions of the Difference Equations}

In this section we will study solutions of the system (5.10) for complex values of $z$.

Let us start the analysis from the description of specific convenient basis in spaces $\operatorname{Inv}_{U_{q}(\mathfrak{g})}\left(V_{\lambda_{0}} \otimes V_{1} \otimes \cdots \otimes V_{N} \otimes\left(V_{\lambda_{N}}\right)^{*}\right)$.

We have isomorphisms of linear spaces:

$$
\operatorname{Inv}_{U_{q}(\mathfrak{g})}\left(V_{\lambda_{0}} \otimes V_{1} \otimes \cdots \otimes V_{N} \otimes\left(V_{\lambda_{N}}\right)^{*}\right) \stackrel{\sim}{\rightarrow} \mathscr{H}_{\lambda_{0}, \lambda_{N}}^{V_{1} \ldots V_{N}} \stackrel{\sim}{\rightarrow} \bigoplus_{\lambda_{1} \ldots \lambda_{N}} \mathscr{H}_{\lambda_{0} \lambda_{1} \ldots \lambda_{N}}^{V_{1} \ldots V_{N}},
$$

where

$$
\mathscr{H}_{\lambda_{0} \ldots \lambda_{N}}^{V_{1} \ldots V_{N}}=\mathscr{H}_{\lambda_{0} \lambda_{1}}^{V_{1}} \otimes \mathscr{H}_{\lambda_{1} \lambda_{2}}^{V_{2}} \otimes \cdots \otimes \mathscr{H}_{\lambda_{N-1} \lambda_{N}}^{V_{N}}
$$

and chains $\lambda_{1}, \ldots, \lambda_{N-1}$ of highest weights are such that there exist nonzero embeddings $V_{\lambda_{i}} \hookrightarrow V_{\lambda_{i+1}} \otimes V_{i+1}$. Denote this composition isomorphism as $\left(\eta_{\lambda_{0} \lambda_{N}}^{V_{1} \ldots V_{N}}\right)^{-1}$. The inverse map is a direct sum

$$
\eta_{\lambda_{0} \lambda_{N}}^{V_{1} \ldots V_{N}}=\bigoplus_{\lambda_{1} \ldots \lambda_{N-1}} \eta_{\lambda_{0} \lambda_{1} \ldots \lambda_{N}}^{V_{1} \ldots V_{N}}
$$

where

$$
\eta_{\lambda_{0} \lambda_{1} \ldots \lambda_{N}}^{V_{1} \ldots V_{N}}: \mathscr{H}_{\lambda_{0} \lambda_{1}}^{V_{1}} \otimes \cdots \otimes \mathscr{H}_{\lambda_{N-1} \lambda_{N}}^{V_{N}} \rightarrow \operatorname{Inv}_{U_{q}(\mathfrak{g})}\left(V_{\lambda_{0}} \otimes V_{1} \otimes \cdots \otimes V_{N} \otimes\left(V_{\lambda_{N}}\right)^{*}\right)
$$

Let $\left\{a_{i}\right\}$ be a basis in $H_{\lambda_{i-1} \lambda_{i}}^{V_{i}}$. Then

$$
\eta_{\lambda_{0} \ldots \lambda_{N}}^{V_{1} \ldots V_{N}}\left(a_{1} \otimes \cdots \otimes a_{N}\right):=\eta_{\lambda_{0} \ldots \lambda_{N}}^{V_{1} \ldots V_{N}}\left(a_{1} \ldots a_{N}\right)
$$

is a basis in $\left.\operatorname{Inv}_{U_{q}(\mathfrak{g})}\left(V_{\lambda_{0}} \otimes V_{1} \otimes \cdots \otimes V_{N}\right) \otimes\left(V_{\lambda_{N}}\right)^{*}\right)$.

According to the factorization (4.19) we represent $R^{V W}(z)$ as

$$
R^{V W}(z)=\tilde{f}_{V W}(z) \cdot \tilde{R}^{V W}(z),
$$

where

$$
\tilde{R}^{V W}(z)=\frac{Q^{V W}(z)}{p^{V W}(z)}, \quad \tilde{f}_{V W}(z)=p_{V W}(z) f_{V W}(z)
$$

and $Q^{V W}(z), p_{V W}(z), f_{V W}(z)$ are defined in Sect. 4 .

Then we can write $\mathscr{F}$ in the factorized form:

$$
\mathscr{F}^{V_{1} \ldots V_{N}}\left(z_{1}, \ldots, z_{N}\right)=\prod_{i<j} G_{i j}\left(\frac{z_{i}}{z_{j}}\right) F^{V_{1} \ldots V_{N}}\left(z_{1}, \ldots, z_{N}\right),
$$


where

$$
G_{i j}\left(z q^{-2(k+g)}\right)=\tilde{f}_{V_{i} V_{j}}\left(z q^{-2(k+g)}\right) G_{i j}(z),
$$

and $F^{V_{1} \ldots V_{N}}\left(z_{1}, \ldots, z_{N}\right)$ satisfies the following system:

$$
T_{j} F^{V_{1} \ldots V_{N}}\left(z_{1}, \ldots, z_{N}\right)=A_{j}^{V_{1} \ldots V_{N}}\left(z_{1}, \ldots, z_{N}\right) F^{V_{1} \ldots V_{N}}\left(z_{1}, \ldots, z_{N}\right) .
$$

Here $z_{1}, \ldots, z_{N} \in \mathbf{C}^{\times}$and

$$
\begin{aligned}
& A_{j}^{V_{1} \ldots V_{N}}\left(z_{1}, \ldots, z_{N}\right) \\
& =\tilde{R}_{j j-1}^{V_{j} V_{j-1}}\left(\frac{z_{j}}{z_{j-1}} p\right) \ldots \tilde{R}_{j_{1}}^{V_{j} V_{1}}\left(\frac{z_{j}}{z_{1}} p\right) \cdot \bar{R}_{j 1,0}^{V_{j}, V_{\lambda_{0}}}\left(q^{2 \varrho}\right)_{j}\left(\bar{R}_{N+1, j}^{V_{\lambda, N}^{*}, V_{j}}\right)^{-1} \\
& \quad \times \tilde{R}_{j N}^{V_{j} V_{n}}\left(\frac{z_{j}}{z_{N}}\right) \ldots \tilde{R}_{j j+1}^{V_{j} V_{j+1}}\left(\frac{z_{j}}{z_{j+1}}\right) .
\end{aligned}
$$

Starting from now we assume that $q \in \mathbf{C}^{\times},|q|<1$, and, for convenience, that $k+g<0$. Then $p=q^{-2(k+g)}$ is such that $|p|<1$. The function $\tilde{f}_{V_{i} V_{j}}(z)$ is an analytic function at $z=0$. Equation (6.2) has a unique solution over $z^{-\frac{\left(\mu_{i}, \mu_{\jmath}\right)}{2(k+g)}} \mathbf{C}[[z]]$, which can be continued from $\mathbf{C}[[q-1]]$ to complex values of $q$ with $|q|<1$. For these values of $q$ this solution is given by the following product:

$$
G_{i j}(z)=z^{-\frac{\left(\mu_{i} \mu_{j}\right)}{2(k+g)}} \prod_{n \geq 1} \tilde{f}_{V_{i} V_{j}}^{0}\left(z p^{n}\right)
$$

where $\mu_{i}$ and $\mu_{j}$ are $U_{q}(\mathfrak{g})$-highest weights of $V_{i}$ and $V_{j}$, respectively, and $\tilde{f}_{V_{i} V_{j}}^{0}(z)=$ $q^{-\left(\mu_{i}, \mu_{\jmath}\right)} \tilde{f}_{V_{\imath} V_{j}}(z)$.

We will study first the system of $q$-difference equations (6.3), (6.4) with rational coefficients. Its solutions differ from the solutions of the original system (5.10) by a product of the scalar functions (6.5). We also consider only $U_{q}(\mathfrak{g})$-invariant solutions, therefore we restrict the system (6.3) to the subspace (6.0). Generally speaking, there exist infinitely many linear independent solutions of this system even if we require the analyticity of solutions in a certain neighborhood of $\mathbf{C}^{N}$. In fact, one can always multiply a given solution by an analytic function invariant with respect to the shift operators (5.6). However, if we fix an asymptotic behavior of solutions at $\left|z_{1}\right| \gg$ $\left|z_{2}\right| \gg \cdots \gg\left|z_{N}\right|$ (or any permutation of the indexes) by considering the limit of the coefficients (6.4) at $\left|\frac{z_{\imath}}{z_{i+1}}\right| \rightarrow \infty$ for $i=1, \ldots, N-1$, we can construct formal solutions in terms of the power series in $\mathbf{C}\left(\left(z_{1}, \frac{z_{2}}{z_{1}}, \ldots, \frac{z_{N}}{z_{N-1}}\right)\right)$ multiplied by an appropriate asymptotic function. By the general theory of $q$-difference equations developed in the case of one variable in [Ad, $\mathrm{Bi}, \mathrm{C}]$ and generalized to several variables in [A1] these formal power series solutions are analytic functions in the domain $\left|z_{1}\right| \gg \cdots \gg\left|z_{N}\right|$. One can then show (see [A1]) that for generic coefficient functions (6.4) these solutions can be extended to meromorphic functions in $\left(\mathbf{C}^{\times}\right)^{N}$. Here generic means that the eigenvalues of functions (6.4) in the limit $\left|\frac{z_{i}}{z_{i+1}}\right| \rightarrow \infty$ for $i=1, \ldots, N-1$, do not differ by an integral power of $p$. In our case of Eqs. (6.3), (6.4), this is guaranteed by the condition $k \notin \mathbf{Q}$ (see Lemma 6.1 below), which we will assume in this section. By the construction, the dimension of the space of solutions 
obtained by the analytic continuation of the formal power series with asymptotics determined by the limit of the coefficient functions is equal to the dimension of the $q$-difference system. This fact justifies the name $q$-holonomic system of difference equations and makes it analogous to holonomic systems of differential equations such as the Knizhnik-Zamolodchikov equation.

In order to describe an asymptotic basis of solutions of (6.3) at $\left|z_{1}\right| \gg\left|z_{2}\right| \gg$ $\cdots \gg\left|z_{N}\right|$, let us consider first asymptotics of $A_{j}(z)$ in this limit. We define

$$
\bar{A}_{j}^{V_{1} \ldots V_{N}}=\bar{R}_{j, j-1}^{V_{\jmath}, V_{j-1}} \ldots \bar{R}_{j, 0}^{V_{\jmath}, V_{0}}\left(q^{2 \varrho}\right)_{j} \cdot\left(\bar{R}_{N+1, j}^{V_{\lambda_{N}}^{*}, V_{j}}\right)^{-1} \ldots\left(\bar{R}_{j+1, j}^{V_{j+1}, V_{\jmath}}\right)^{-1} q^{m_{\jmath}(N, 1)}
$$

where $m_{j}(N, 1)=\left(\mu_{j}, \mu_{N}+\cdots+\mu_{j+1}-\mu_{j-1}-\cdots-\mu_{1}\right)$ and $\mu_{j}$ are $U_{q}(\mathfrak{g})$-highest weight of $V_{j}$ regarded as $U_{q}(\mathfrak{g})$-module. Clearly, if $\left|\frac{z_{i}}{z_{i+1}}\right| \rightarrow \infty$ for $i=1, \ldots, N-1$,
we have:

$$
A_{j}^{V_{1} \ldots V_{N}}\left(z_{1} \ldots z_{N}\right) \rightarrow \bar{A}_{j}^{V_{1} \ldots V_{N}}
$$

\section{Lemma 6.1.}

$$
\bar{A}_{j}^{V_{1} \ldots V_{N}} \cdot \eta_{\lambda_{0} \ldots \lambda_{N}}^{V_{1} \ldots V_{N}}\left(a_{1}, \ldots, a_{N}\right)=q^{C\left(\lambda_{j}\right)-C\left(\lambda_{j-1}\right)} \eta_{\lambda_{0} \ldots \lambda_{N}}^{V_{1} \ldots V_{N}}\left(a_{1}, \ldots, a_{N}\right) \cdot q^{m_{j}(N, 1)}
$$

Proof. This lemma follows from the formula for the action of the comultiplication on $R$-matrices and from Theorem 3.2 combined with the definition of $\eta$-basis.

Now we can construct a basis for the solutions of the asymptotical system obtained from the system (6.3) in the limit $\left|\frac{z_{1}}{z_{i+1}}\right| \rightarrow \infty$ for $i=1, \ldots, N-1$,

$$
T_{j} \bar{F}^{V_{1} \ldots V_{N}}\left(z_{1} \ldots z_{N}\right)=\bar{A}_{j}^{V_{1} \ldots V_{N}} \bar{F}^{V_{1} \ldots V_{N}}\left(z_{1} \ldots z_{N}\right)
$$

Definition 6.1. Let $(\lambda, a)=\left(\left(\lambda_{0}, \lambda_{1}, \ldots, \lambda_{N}\right),\left(a_{1}, \ldots, a_{N}\right)\right)$ be as in (6.1). We define $\bar{F}_{(\lambda, a)}^{V_{1} \ldots V_{N}}$ as a solution of the system (6.7) such that

$$
\bar{F}_{(\lambda, a)}^{V_{1} \ldots V_{N}}=\prod_{i=1}^{N} z_{i}^{h\left(\lambda_{i-1}\right)-h\left(\lambda_{\imath}\right)-\frac{m(N, 1)}{2(k+g)}} \eta_{\lambda_{0} \ldots \lambda_{N}}^{V_{1} \ldots V_{N}}\left(a_{1} \ldots a_{N}\right)
$$

It is clear that these solutions form a basis in $\operatorname{Inv}_{U_{q}(\mathfrak{g})}\left(V_{\lambda_{0}} \otimes V_{1} \otimes \cdots \otimes V_{N} \otimes\left(V_{\lambda_{N}}\right)^{*}\right)$. that

Definition 6.2. Let $F_{(\lambda, a)}^{V_{1} \ldots V_{N}}\left(z_{1}, \ldots, z_{N}\right)$ be a solution of the system (6.3) such

$$
F_{(\lambda, a)}^{V_{1} \ldots V_{N}}\left(z_{1}, \ldots, z_{N}\right) \rightarrow \bar{F}_{(\lambda, a)}^{V_{1} \ldots V_{N}}\left(z_{1}, \ldots, z_{N}\right)
$$

at $|z| \gg\left|z_{2}\right| \gg \cdots \gg\left|z_{N}\right|$.

Proposition 6.1. (1) Solutions $\mathscr{F}_{(\lambda, a)}^{V_{1} \ldots V_{N}}$ form an asymptotic basis of solutions of (6.3) at $\left|\frac{z_{i}}{z_{i+1}}\right| \rightarrow \infty, i=1, \ldots, N-1$. 
(2) Let $\left|\frac{z_{i}}{z_{i+1}}\right| \rightarrow \infty$ for a fixed index $i$. In this limit, the basis $F_{(\lambda, a)}\left(z_{1} \ldots z_{N}\right)$ has the following factorization property:

$$
\begin{aligned}
& \left(F_{\left(\lambda_{0}, \ldots, \lambda_{N}\right)\left(a_{1} \ldots a_{N}\right)}^{V_{1} \ldots V_{N}}\left(z_{1}, \ldots, z_{N}\right)\right) \\
& \quad \times\left(F_{\left(\lambda_{0}, \ldots, \lambda_{\imath}\right)\left(a_{1}, \ldots, a_{\imath}\right)}^{V_{1} \ldots V_{N}}\left(z_{1}, \ldots, z_{i}\right)\right. \\
& \quad \times\left(F_{\left(\lambda_{1}, \ldots, \lambda_{N}\right)\left(a_{\imath+1} \ldots z_{N}\right)}^{V_{1} \ldots V_{N}}\left(z_{i+1} \ldots z_{N}\right)\right) \\
& \quad \times \prod_{j=1}^{i} z_{j}^{-h_{j}(i+1, N)} \cdot \prod_{j=i+1}^{N} z_{j}^{h_{\jmath}(1, i)}
\end{aligned}
$$

where $h_{j}(k, l)=\frac{1}{2(k+g)}\left(\mu_{j}, \mu_{k}+\mu_{k+1}+\cdots+\mu_{l}\right)$ for $k \leq l$.

Proof. Let $\left(A_{j}\left(z_{1} \ldots z_{N}\right)\right)_{\left(\lambda^{\prime}, a^{\prime}\right)}^{(\lambda, a)}$ be matrix coefficients of $A_{j}$ in the $\eta$-basis:

$$
A_{\jmath}\left(z_{1} \ldots z_{N}\right) \eta_{(\lambda, a)}=\sum_{\left(\lambda^{\prime}, a^{\prime}\right)}\left(A_{j}\left(z_{1} \ldots z_{N}\right)\right)_{(\lambda, a)}^{\left(\lambda^{\prime}, a^{\prime}\right)} \eta_{\left(\lambda^{\prime}, a^{\prime}\right)}
$$

Using the formula for the action of the comultiplication on $\bar{R}$ and the explicit form of $A_{j}$ we obtain the following factorization of these matrix elements when $\left|\frac{z_{i}}{z_{i+1}}\right| \rightarrow \infty:$ for $j \leq i$

$$
\begin{aligned}
& \left(A_{j}\left(z_{1}, \ldots, z_{N}\right)\right)_{\left(\lambda^{\prime}, a^{\prime}\right)}^{(\lambda, a)} \rightarrow\left(A_{j}\left(z_{1} \ldots z_{i}\right)\right)_{\left(\lambda_{0}^{\prime}, \ldots, \lambda_{2}^{\prime}\right)\left(a_{1}^{\prime} \ldots a_{\imath}^{\prime}\right)}^{\left(\lambda_{0}, \ldots, \lambda_{2}\right)\left(a_{1} \ldots a_{2}\right)} \delta_{\lambda_{0} \lambda_{0}^{\prime}} \\
& \quad \times \prod_{l=i}^{N} \delta_{\lambda_{l} \lambda_{l}^{\prime}} \delta_{a_{l} a_{l}^{\prime}} \cdot q^{\left(\mu_{j}, \mu_{N}+\cdots+\mu_{i+1}\right)}
\end{aligned}
$$

for $j \geq i$,

$$
\begin{aligned}
\left(A_{j}\left(z_{1}, \ldots, z_{N}\right)\right)_{\left(\lambda^{\prime}, a^{\prime}\right)}^{(\lambda, a)}= & \left(A_{j}\left(z_{i}, \ldots, z_{N}\right)\right)_{\left(\lambda_{i}^{\prime}, \ldots, \lambda_{N}\right)\left(a_{i+1}^{\prime} \ldots a_{N}^{\prime}\right)}^{\left(\lambda_{i}, \ldots, \lambda_{N}\right)\left(a_{i+1} \ldots a_{N}\right)} \\
& \times \prod_{l=0}^{i} \delta_{\lambda_{l} \lambda_{l}^{\prime}} \prod_{l=1}^{i} \delta_{a_{l} a_{l}^{\prime}} \cdot \delta_{\lambda_{N} \lambda_{N}^{\prime}} \cdot q^{-\left(\mu_{\jmath}, \mu_{1}+\cdots+\mu_{\imath}\right)} .
\end{aligned}
$$

Together with the definition of $F_{(\lambda, a)}$-basis of solutions of (6.3) this gives the factorization.

Theorem 6.1. Let $S_{N}$ be a symmetric group of order $N$ and let $w \in S_{N}$. Solutions $F_{(\lambda, a)}^{V_{1} \ldots V_{N}}$ can be analytically continued to $\left|z_{w_{1}}\right| \gg \cdots \gg\left|z_{w_{N}}\right|$ for any $w$ and they form a basis of linearly independent solutions in each of such regions.

Proof. Our holonomic system (with matrices of coefficients $A_{j}\left(z_{1}, \ldots, z_{N}\right)$ ) satisfies the conditions of Lemma (6.1). It follows from [A1] that under these conditions the solution of the holonomic $q$-difference equation with rational functions $A_{j}\left(z_{1}, \ldots, z_{N}\right)$ with asymptotics (6.8) can be analytically continued into the region $\left|z_{w_{(1)}}\right| \gg \cdots \gg\left|z_{w_{(N)}}\right|$.

Another basis of solutions of (6.3) in the asymptotic regions $\left|z_{w_{(1)}}\right| \gg \cdots \gg$ $\left|z_{w_{(N)}}\right|$ can be obtained from the following symmetry of $A_{j}^{V_{1} \ldots V_{N}}\left(z_{1} \ldots z_{N}\right)$. 
Proposition 6.2. Let $\check{R}_{i j}^{V_{i} V_{j}}\left(z_{i} / z_{j}\right)=P_{i j}^{V_{i} V_{j}} R_{i j}^{V_{\imath} V_{j}}\left(\frac{z_{i}}{z_{j}}\right)$. The following relations hold

$$
\begin{aligned}
& \check{R}_{i i+1}^{V_{i} V_{\imath+1}}\left(\frac{z_{i}}{z_{i+1}}\right) A_{j}^{V_{1} \ldots V_{i} V_{i+1} \ldots V_{N}}\left(\ldots z_{i}, z_{i+1} \ldots\right) \\
& =A_{j}^{V_{1} \ldots V_{i+1} V_{i} \ldots V_{N}}\left(\ldots z_{i+1}, z_{i} \ldots\right) \tilde{R}_{i i+1}^{V_{i} V_{i+1}}\left(\frac{z_{i}}{z_{i+1}}\right) \\
& \check{R}_{j-1 j}^{V_{j-1} V_{j}}\left(\frac{z_{j-1}}{z_{j} p}\right) A_{j}^{V_{1} \ldots V_{j-1} V_{j} \ldots V_{N}}\left(\ldots z_{j-1}, z_{j} \ldots\right) \\
& =A_{j-1}^{V_{1} \ldots V_{j} V_{j-1} \ldots V_{N}}\left(\ldots z_{j}, z_{j-1} \ldots\right) \check{R}_{j-1}^{V_{j-1} V_{j}}\left(\frac{z_{j-1}}{z_{j}}\right) \\
& \check{R}_{j j+1}^{V_{j} V_{j+1}}\left(\frac{z_{j} p}{z_{j+1}}\right) A_{j}^{V_{1} \ldots V_{j} V_{j+1} \ldots V_{N}}\left(\ldots z_{j}, z_{j+1} \ldots\right) \\
& =A_{j+1}^{V_{1} \ldots V_{j+1} V_{j} \ldots V_{N}}\left(\ldots z_{j+1}, z_{j} \ldots\right) \check{R}_{j j+1}^{V_{j} V_{j+1}}\left(\frac{z_{j}}{z_{j+1}}\right) .
\end{aligned}
$$

Proof. The relation (6.11) follows from the Yang-Baxter equation, others are corollaries of (4.20).

For a given vector spaces $\left\{V_{i}\right\}_{i=1}^{N}, w \in S_{N}$ and

$$
\Omega_{w}=\left\{\left(z_{1}, \ldots, z_{N}\right) \in\left(\mathbf{C}^{\times}\right)^{\otimes N}\left|\frac{z_{i}}{z_{j}} \neq q^{n} p^{m}, n, m \in \mathbf{Z},\right| z_{w_{(\imath)}}|>| z_{w_{(\imath+1)}} \mid\right\}
$$

let $[\tau]_{w}, \tau, w \in S_{N}$, be a space of functions $F: \Omega_{w} \rightarrow V_{\tau_{(1)}} \otimes \cdots \otimes V_{\tau_{(N)}}$. For a simple transposition $s_{i} \in S_{N}$ we define a map $R_{s_{i}}:[\tau]_{w} \rightarrow\left[s_{i} \tau\right]_{s_{i} w}$ such that

$$
\begin{aligned}
R_{s_{i}} & \left(F^{V_{\tau(1)} \ldots V_{\tau(N)}}\left(z_{1}, \ldots, z_{N}\right)\right) \\
& =\check{R}_{i i+1}^{V_{\tau(i)} V_{\tau(i+1)}}\left(\frac{z_{i}}{z_{i+1}}\right) F^{V_{\tau(1)} \ldots V_{\tau(N)}}\left(z_{1}, \ldots, z_{N}\right),
\end{aligned}
$$

where $\breve{R}_{i i+1}^{V_{\tau(i)} V_{\tau(i+1)}}(z)=P_{i i+1}^{V_{\tau(i)} V_{\tau(i+1)}}(z)$.

For any $\sigma \in S_{N}$ let $\sigma=s_{i_{l}} \ldots s_{i_{1}}$ be a minimal decomposition of $\sigma$ in the product of simple transposition. We define

$$
R_{\sigma}=R_{s_{i_{l}}} \ldots R_{s_{i_{1}}} .
$$

Proposition 6.3. The operators $R_{\sigma}, \sigma \in S_{N}$, are well defined and the map $\sigma \mapsto R_{\sigma}$ is a representation of the symmetric group $S_{N}$.

Proof. Relations $R_{s_{\imath}}^{2}=1$ follows from (4.20), other relations follow from the YangBaxter relation for $R^{V_{i} V_{j}}(z)$ and from the definition of $R_{s_{i}}$. The $S_{N}$-relations imply that the definition of $R_{\sigma}$ does not depend on a decomposition.

Proposition 6.4. If $F \in[\tau]_{w}$ and if it is a solution of the system

$$
\begin{aligned}
& T_{w_{(i)}} F^{V_{\tau(1)} \ldots V_{\tau(N)}}\left(z_{w_{(1)}}, \ldots, z_{w_{(N)}}\right) \\
& \quad=A_{w_{i}}^{V_{\tau_{(1)} \ldots V_{\tau_{(N)}}}}\left(z_{w_{(1)}}, \ldots, z_{w_{(N)}}\right) \mathscr{F}^{V_{\tau_{(1)}} \ldots V_{\tau_{(N)}}}\left(z_{w_{(1)}}, \ldots, z_{w_{(N)}}\right),
\end{aligned}
$$

then $R_{\sigma} F \in[\sigma \tau]_{\sigma \omega}$ is a solution of the system 6.15 with $\omega \mapsto \sigma \omega, \tau \mapsto \sigma \tau$.

Proof. The statement is a corollary of Proposition 6.2. 
Therefore, if we have a basis for solutions to the system (6.3) for $\left(z_{1}, \ldots, z_{N}\right) \in$ $\Omega_{1}$ we can continue it to other sectors $\Omega_{\omega}$ in two ways. First, we can use Theorem 6.1 and continue it analytically. Second, we can use Proposition 6.4. Bases obtained in these two ways will be different. We will call the matrix, which transforms one basis into another (according to [Bi]) the connection matrix.

Let $F_{(\lambda, a)}^{\left(V_{\tau_{(i)}} \ldots V_{\left.\tau_{(N)}\right)}\right)}\left(z_{1}, \ldots, z_{N}\right)$ be a basis for solutions to $(6.3)$ in $[\tau]_{1}$ define the elementary connection matrix $\left(\left(C_{i}^{\tau}\left(z_{1} \ldots z_{N}\right)\right)_{(\lambda, a)}^{\left(\lambda^{\prime}, a^{\prime}\right)}\right)$ as the following connection matrix:

$$
\begin{aligned}
R_{s_{\imath}} F^{\left(V_{\tau_{(1)}} \ldots V_{\tau_{(N)}}\right)}\left(z_{1}, \ldots, z_{N}\right)= & \sum_{\left(\lambda^{\prime}, a^{\prime}\right)} F_{\left(\lambda^{\prime}, a^{\prime}\right)}^{\left(V_{\tau_{(1)}} \ldots V_{r_{(i+1)}} V_{\tau_{(2)}} \ldots V_{\left.\tau_{(N)}\right)}\right)}\left(\ldots z_{i+1}, z_{1} \ldots\right) \\
& \times\left(\tilde{C}_{i}^{\tau}\left(z_{1}, \ldots, z_{N}\right)\right)_{(\lambda, a)}^{\left(\lambda^{\prime}, a^{\prime}\right)}
\end{aligned}
$$

Note that here $(\lambda, a)$ is a sequence corresponding to the set $\left(V_{\tau_{(1)}}, \ldots, V_{\tau_{(N)}}\right)$ and $\left(\lambda^{\prime}, a^{\prime}\right)$ is the sequence corresponding to $\left(\ldots V_{\tau_{(i+1)}}, V_{\tau_{(i)}} \ldots\right)$.

Theorem 6.2. The elementary connection matrix $\tilde{C}_{i}^{\tau}$ is a function of $z_{i} / z_{i+1}$ and does not depend on other z-arguments:

$$
\tilde{C}_{i}^{\tau}\left(z_{1} \ldots z_{N}\right)=\tilde{C}_{i}^{\tau}\left(\frac{z_{i}}{z_{i+1}}\right)
$$

and it acts "locally" on the basis $F_{(\lambda, a)}$ :

$$
\begin{aligned}
\left(\tilde{C}_{i}^{\tau}(z)\right)_{(\lambda, a)}^{\left(\lambda^{\prime}, a^{\prime}\right)}= & \tilde{W}^{V_{\tau_{(i)}} V_{\tau_{(i+1)}}}\left[\begin{array}{ccc}
a_{i-1}^{\prime} & \lambda_{i}^{\prime} & a_{i}^{\prime} \\
\lambda_{i-1} & & \lambda_{i+1} \\
a_{i-1} & \lambda_{i} & a_{i}
\end{array}\right] \\
& \times \prod_{\substack{j \neq i, i-1 \\
j=1}}^{N} \delta_{a_{j} a_{j}^{\prime}} \cdot \prod_{\substack{k \neq i \\
k=0}}^{N} \delta_{\lambda_{k} \lambda_{k}^{\prime}}
\end{aligned}
$$

Here $(\lambda, a)$ is a set corresponding to $\left(V_{\tau_{(1)}}, \ldots, V_{\tau_{(N)}}\right)$ and $\left(\lambda^{\prime}, a^{\prime}\right)$ is a set corresponding to $\left(V_{\sigma_{(1)}}, \ldots, V_{\sigma_{(N)}}\right)$ where $\sigma=s_{i} t$.

Proof. The connection matrix $C_{i}^{\tau}$ can depend only on the ratios $\frac{z_{1}}{z_{2}}, \ldots, \frac{z_{i}}{z_{i+1}}, \ldots$, $\frac{z_{N-1}}{z_{N}}$. From the factorization property (6.10) we conclude that it does not depend on $\frac{z_{j}}{z_{j+1}}$ with $j \neq i$, "locality" of $C_{i}^{\tau}$ also follows immediately from this factorization property.

We can reformulate the statement of Theorem 6.2 in a more invariant language. We proved that the connection matrix $\tilde{C}_{i}^{\tau}(z)$ is a linear map:

$$
\begin{gathered}
\tilde{C}_{i}^{\tau}(z): \mathscr{H}_{\lambda_{0} \lambda_{N}}^{V_{\tau(1) \ldots V_{\tau(i)}} V_{\tau(i+1) \cdots V_{\tau(N)}}} \rightarrow \mathscr{H}_{\lambda_{0} \lambda_{N}}^{V_{\tau(1) \cdots V_{\tau(i+1)}} V_{\tau(i) \cdots V_{\tau(N)}}} \\
\tilde{C}_{i}^{\tau}(z)=1 \otimes \cdots \otimes \tilde{W}^{V_{\tau(i)} V_{\tau(i+1)}}(z) \otimes \cdots \oplus 1
\end{gathered}
$$


Here the linear map

$$
\begin{gathered}
\tilde{W}^{V_{\tau(i)} V_{\tau(i+1)}}(z): \bigoplus_{\lambda_{i}} \mathscr{H}_{\lambda_{\imath-1}, \lambda_{1}}^{V_{\tau(i)}} \otimes \mathscr{H}_{\lambda_{\imath} \lambda_{i+1}}^{V_{\tau(i+1)}} \\
\rightarrow \bigoplus_{\lambda_{\imath}^{\prime}} \mathscr{H}_{\lambda_{i-1} \lambda_{i}^{\prime}}^{V_{\tau(i+1)}} \otimes \mathscr{H}_{\lambda_{\imath}^{\prime} \lambda_{i+1}}^{V_{\tau(i+1)}}
\end{gathered}
$$

is given by

$$
\tilde{W}^{V_{\tau(\imath)} V_{\tau(i+1)}}(z)=\bigoplus_{\lambda_{i}} \bigoplus_{\lambda_{i}^{\prime}} \tilde{W}^{V_{\tau(\imath)} V_{\tau(i+1)}}\left[\begin{array}{ccc} 
& \lambda_{i-1} & \\
\lambda_{i}^{\prime} & & \lambda_{i} \\
& \lambda_{i+1} &
\end{array}\right](z),
$$

where

$$
\tilde{W}^{V_{\tau(i)} V_{\tau(i+1)}}\left[\begin{array}{ccc} 
& \lambda_{i-1} & \\
\lambda_{i}^{\prime} & & \lambda_{i} \\
& \lambda_{i+1}
\end{array}\right](z): \mathscr{H}_{\lambda_{i-1} \lambda_{1}}^{V_{\tau(i)}} \otimes \mathscr{H}_{\lambda_{i} \lambda_{i+1}}^{V_{\tau(i+1)}} \rightarrow \mathscr{H}_{\lambda_{2-1} \lambda_{i}^{\prime}}^{V_{\tau\left(i_{1}\right)}} \otimes \mathscr{H}_{\lambda_{i}^{\prime} \lambda_{i+1}}^{V_{\tau(i)}}
$$

in a specific basis admits a form as in (6.18):

$$
a_{i} \otimes a_{i+1} \mapsto \sum_{a_{\imath}^{\prime} a_{i+1}^{\prime}} \tilde{W}^{V_{\tau_{(i)}} V_{\tau}}\left[\begin{array}{ccc}
a_{i-1}^{\prime} & \lambda_{i}^{\prime} & a_{i}^{\prime} \\
\lambda_{i-1} & & \lambda_{i+1} \\
a_{i-1} & \lambda_{i} & a_{i}
\end{array}\right](z) \cdot a_{i+1}^{\prime} \otimes a_{i}^{\prime},
$$

where $a_{i}, a_{i+1}, a_{i+1}^{\prime}, a_{i}^{\prime}$ are bases in $\mathscr{H}_{\lambda_{\imath-1} \lambda_{i}}^{V_{\tau(i)}}, \mathscr{H}_{\lambda_{i} \lambda_{i+1}}^{V_{\tau(i+1)}}, \mathscr{H}_{\lambda_{\imath-1} \lambda_{i}^{\prime}}^{V_{\tau(i+1)}}$, and in $\mathscr{H}_{\lambda_{i}^{\prime} \lambda_{i+1}}^{V_{\tau(i)}}$, respectively.

Theorem 6.3. Elementary connection matrices satisfy the following relations:

$$
\begin{gathered}
\tilde{C}_{i}^{s_{i+1} s_{i} \tau}(w) \tilde{C}_{i+1}^{s_{i} \tau}(z w) \tilde{C}_{i}^{\tau}(z)=\tilde{C}_{i+1}^{s_{i} s_{i+1} \tau}(z) \tilde{C}_{i}^{s_{i+1} \tau}(z w) \tilde{C}_{i+1}^{\tau}(w) \\
\tilde{C}_{i}^{s_{i} \tau}\left(z^{-1}\right) \tilde{C}_{i}^{\tau}(z)=I_{\mathscr{H}} .
\end{gathered}
$$

Here $I_{\mathscr{H}}$ is the identical operator in $\mathscr{H}_{\lambda_{0} \lambda_{N}}^{V_{\tau(1) \ldots V_{\tau(N)}}}(\tau)$.

Proof. Both of these relations follow from Proposition 6.3.

A remarkable consequence of the locality (6.19) of connection matrices is that we can compute them from the analysis of a two point correlation function $F^{V_{1} V_{2}}\left(z_{1}, z_{2}\right)$. Therefore, let us consider the case of two point correlation functions in more detail.

Proposition 6.5. Let $F_{(\lambda, a)}$ be a basis of solutions for the system (6.3) described above then for $N=2$,

$$
F_{(\lambda, a)}\left(z_{1}, z_{2}\right)=\left(z_{1} z_{2}\right)^{\frac{h\left(\lambda_{2}\right)-h\left(\lambda_{0}\right)}{2}} \varphi_{(\lambda, a)}\left(\frac{z_{1}}{z_{2}}\right) .
$$

Proof. From (6.3) and (4.20) we have:

$$
\left(T_{1} T_{2}\right) F\left(z_{1}, z_{2}\right)=\bar{R}_{12}^{V_{1} V_{\lambda_{0}}}\left(q^{2 \varrho}\right)_{1} \bar{R}_{13}^{V_{1}\left(V_{\lambda_{2}}\right)^{*}} \bar{R}_{20}^{V_{2} V_{\lambda_{0}}}\left(q^{2 \varrho}\right)_{2} \bar{R}_{23}^{V_{2}\left(V_{\lambda_{2}}\right)^{*}} F\left(z_{1}, z_{2}\right) .
$$

Since $F\left(z_{1}, z_{2}\right) \in \operatorname{Inv}_{U_{q}(\mathfrak{g})}\left(V_{\lambda_{0}} \otimes V_{1} \otimes V_{2} \otimes\left(V_{\lambda_{2}}\right)^{*}\right)$, it follows from Theorem 3.2 that

$$
\left(T_{1} T_{2}\right) F_{(\lambda, a)}\left(z_{1}, z_{2}\right)=q^{C\left(\lambda_{2}\right)-C\left(\lambda_{0}\right)} F_{(\lambda, a)}\left(z_{1}, z_{2}\right) \text {. }
$$


This implies

$$
\left(T_{1} T_{2}\right) \varphi_{(\lambda, a)}\left(z_{1}, z_{2}\right)=\varphi_{(\lambda, a)}\left(z_{1}, z_{2}\right),
$$

where $\varphi$ is given by (6.24). From the definition of $F$, we conclude that

$$
\varphi_{(\lambda, a)}\left(z_{1}, z_{2}\right) \in\left(\frac{z_{1}}{z_{2}}\right)^{\frac{h\left(\lambda_{0}\right)+h\left(\lambda_{2}\right)-2 h\left(\lambda_{1}\right)-2 h_{1}(2,2)}{2}} \mathbf{C}\left(\left(\frac{z_{2}}{z_{1}}\right)\right),
$$

and therefore $\varphi_{(\lambda, a)}\left(z_{1}, z_{2}\right)=\varphi_{(\lambda, a)}\left(\frac{z_{1}}{z_{2}}\right)$. Remember that $h_{1}(2,2)=\frac{\left(\mu_{1}, \mu_{2}\right)}{2(k+g)}$ and $\mu_{i}$ is a $U_{q}(\mathfrak{g})$ highest weight of $V_{i}$.

So, the functions $\varphi_{(\lambda, a)}(z)$ form a basis for solutions to the system

$$
T \varphi(z)=q^{\frac{C\left(\lambda_{0}\right)-C\left(\lambda_{2}\right)}{2}} \bar{R}_{10}^{V_{1} V_{\lambda_{0}}}\left(q^{2 \varrho}\right)_{1} \bar{R}_{13}^{V_{1}\left(V_{\lambda_{2}}\right)^{*}} \tilde{R}_{12}(z) \varphi(z)
$$

and

$$
\varphi_{\left(\lambda_{0} \lambda_{1} \lambda_{2}\right)\left(a_{1} a_{2}\right)}(z) \in z^{\frac{h\left(\lambda_{0}\right)+h\left(\lambda_{2}\right)-2 h\left(\lambda_{1}\right)-2 h_{1}(2,2)}{2}} \mathbf{C}\left(\left(\frac{1}{z}\right)\right) .
$$

The general theory of linear $q$-difference equations has been studied in [Ad, Bi, C] (and references therein). In particular, in the case when the coefficients are rational functions bounded at $z=0$ and $z=\infty$, the solutions to the difference equations are well defined meromorphic functions in the complex plane with possible essential singularities at $z=0$ and $z=\infty$. We can apply this general theory to the equation (6.25).

First consider an example of scalar difference equation (we had such an example earlier in Sect. 4):

$$
\varphi(z p)=\frac{1-z p^{\alpha}}{1-z p^{\beta}} \varphi(z) .
$$

There exist a unique solution to this equation in $\mathbf{C}[[z]]$ :

$$
\varphi_{0}(z)=\frac{\left(z p^{\beta} ; p\right)_{\infty}}{\left(z p^{\alpha} ; p\right)_{\infty}}
$$

and a unique solution over $z^{\alpha-\beta} \mathbf{C}\left[\left[z^{-1}\right]\right]$ :

$$
\varphi_{\infty}(z)=z^{\alpha-\beta} \frac{\left(z^{-1} p^{-\alpha+1} ; p\right)_{\infty}}{\left(z^{-1} p^{-\beta+1} ; p\right)_{\infty}}
$$

where

$$
(z ; p)_{\infty}=\prod_{n \geq 0}\left(1-z p^{n}\right) .
$$

Clearly, $\varphi_{0}(z)$ can be continued for $|p|<1$ to a meromorphic function on $\mathbf{C}$ with essential singularity at $z=\infty$. In the same way $z^{\beta-\alpha} \varphi_{\infty}(z)$ can be continued to the meromorphic function on $\mathbf{C}^{\times}$, regular at $z=\infty$ with essential singularity at $z=0$. The ratio

$$
\tilde{C}(z)=\frac{\varphi_{0}(z)}{\varphi_{\infty}(z)}
$$

is called the connection matrix and (6.26), (6.27) imply that

$$
\tilde{C}(z)=z^{\alpha-\beta} \frac{\Theta\left(z p^{\alpha}\right)}{\Theta\left(z p^{\beta}\right)}
$$


where

$$
\Theta(z)=\prod_{n \geq 0}\left(1-p^{n+1}\right)\left(1-z p^{n}\right)\left(1-z^{-1} p^{n+1}\right)
$$

is the Jacobi elliptic theta function. Since

$$
\tilde{C}(z p)=\tilde{C}(z)
$$

such functions are also called pseudoconstants [Bi].

Now we return to a nonscalar case of (6.25). As it follows from the analysis given in the beginning of this section there exist two bases of fundamental solutions to this equation

$$
\begin{gathered}
\varphi_{(\lambda, a)}^{V_{1} V_{2}}(z)=z^{\frac{h\left(\lambda_{0}\right)+h\left(\lambda_{2}\right)-2 h\left(\lambda_{1}\right)}{2}-h_{1}(2,2)}\left(\varphi_{(\lambda)(a)}^{V_{1} V_{2}}(z)\right)_{\infty} \\
\tilde{R}_{21}\left(z^{-1}\right) \varphi_{\left(\lambda^{\prime}, a^{\prime}\right)}^{V_{2} V_{1}}\left(z^{-1}\right)=z^{\frac{h\left(\lambda_{0}\right)+h\left(\lambda_{2}\right)-2 h\left(\lambda_{1}^{\prime}\right)}{2}+h_{1}(2,2)}\left(\varphi_{\left(\lambda^{\prime}\right)\left(a^{\prime}\right)}^{V_{1} V_{2}}(z)\right)_{0} .
\end{gathered}
$$

Here $\left(\varphi_{(\lambda)(a)}^{V_{1} V_{2}}(z)\right)_{\infty}$ is a regular function at $z=\infty$, and $\left(\varphi_{\left(\lambda^{\prime}, a^{\prime}\right)}^{V_{1} V_{2}}(z)\right)_{0}$ regular at $z=0$.

Let us write these fundamental solutions simply as $z^{\beta_{i}}\left(\varphi_{i}^{V_{1} V_{2}}(z)\right)_{\infty}$ and $z^{\alpha_{i}}\left(\varphi_{i}^{V_{1} V_{2}}(z)\right)_{0}$, respectively.

Since coefficients $\tilde{R}^{V_{1} V_{2}}(z)$ of the system are rational functions regular at $z=0, \infty$ we can analytically continue both these solutions to meromorphic functions [Bi]. We can continue $\left(\varphi_{i}^{V_{1} V_{2}}(z)\right)_{0}$ to $\mathbf{C}$ and $\left(\varphi_{i}^{V_{1} V_{2}}(z)\right)_{\infty}$ to $\mathbf{C}^{\times}$. Therefore, for finite values of $z$ there must exist the connection matrix

$$
z^{\alpha_{\imath}}\left(\varphi_{i}^{V_{1} V_{2}}(z)\right)_{0}=\sum_{j} \tilde{C}_{i j}(z) z^{\beta_{i}}\left(\varphi_{j}^{V_{1} V_{2}}(z)\right)_{\infty},
$$

where the coefficients $\tilde{C}_{i j}(z)$ are pseudoconstants (6.28). The results of [Bi], part IV, implies

Proposition. The coefficients of the connection matrix $\tilde{C}_{i j}(z)$ have the form

$$
\tilde{C}_{i j}(z)=c_{i j} z^{\alpha_{i}-\beta_{j}} \frac{\Theta\left(z p^{a_{\imath \jmath}^{1}}\right) \ldots \Theta\left(z p^{a_{i j}^{m}}\right)}{\Theta\left(z p^{b_{i j}^{1}}\right) \ldots \Theta\left(z p^{b_{\imath \jmath}^{m}}\right)},
$$

where $a_{i j}^{r}, b_{i j}^{r}, c_{i j}$ are constants and

$$
\sum_{r=1}^{m}\left(a_{i j}^{r}-b_{i j}^{r}\right)=\alpha_{i}-\beta_{j} .
$$

Thus the connection matrices for the system (6.3) satisfy the Yang-Baxter equation and the unitarity (6.22), (6.23) and matrix elements of these matrices have the form (6.32). In fact, as it follows from the explicit form of chosen basis for solutions, we can "gauge out" power functions form matrix elements of connection matrix. Indeed, from (6.31), we conclude that

$$
\left.\left.\left(\varphi_{\imath}^{V_{1} V_{2}}(z)\right)_{0}=\sum_{j} C_{i j}(z)\right) \varphi_{j}^{V_{1} V_{2}}(z)\right)_{\infty}
$$

where $C_{i j}(z)$ is a ratio of theta functions. Since elementary connection matrices are determined by two-point function connection matrix, we proved the following theorem. 
Theorem 6.4. Let $F_{(\lambda, a)}^{V_{1} V_{2}}$ be a basis to solutions of (6.8) described above, then

$$
\begin{aligned}
R_{s_{\imath}} & F_{(\lambda, a)}^{V_{\tau(1)} \ldots V_{\tau(N)}}\left(z_{1}, \ldots, z_{N}\right) \prod_{i=1}^{N} z_{i}^{h\left(\lambda_{i-1}\right)-h\left(\lambda_{\imath}\right)-\frac{m_{i}(N, 1)}{2(k+g)}} \\
= & \sum_{\left(\lambda^{\prime}, a^{\prime}\right)} F_{\left(\lambda^{\prime}, a^{\prime}\right)}^{V_{\tau(1)} \ldots V_{\tau(i+1)} V_{\tau(\imath)} \ldots V_{\tau(N)}}\left(z_{1}, \ldots, z_{i+1}, z_{i}, \ldots, z_{N}\right) \\
& \times \prod_{i=1}^{N} z_{i}^{h\left(\lambda_{i+1}^{\prime}\right)-h\left(\lambda_{i}^{\prime}\right)-\frac{m_{i}(N, 1)}{2(k+g)}-\frac{\left(\mu_{i}, \mu_{j}\right)}{k+g}}\left(C_{i}^{\tau}\left(z_{i} / z_{i+1}\right)\right)_{(\lambda, a)}^{\left(\lambda^{\prime}, a^{\prime}\right)}
\end{aligned}
$$

where matrix elements of $C_{i}^{\tau}(z)$ are ratios of theta functions and $C_{i}^{\tau}(z)$ satisfies Eqs. (6.22) and (6.23).

Elliptic solutions to the Yang-Baxter equation were constructed for all classical types of $\mathfrak{g}$, when $V_{i}$ are vector representations, in a series of papers [Bel, JKMO, JMO] which are far reaching generalizations of the first solution of these equations by Baxter. For $\mathfrak{g}=\mathfrak{s l}_{n}$ series of solutions were also constructed corresponding to symmetrical and antisymmetrical powers of vector representations (DJKMO, DJMO]. All such known solutions are expressed in terms of ratios of theta functions. So, we propose the following conjecture.

Conjecture 1. All unitary solutions to the Yang-Baxter equation obtained in [DJMO, $\mathrm{JMO}, \mathrm{JKMO}]$ are equivalent to solutions given by the connection matrix $C(z)$ for the appropriate type of a simple Lie algebra $\mathfrak{g}$ and finite-dimensional representations of $U_{q}(\hat{\mathfrak{g}})$.

Now we consider functions $\mathscr{F} V_{1} \ldots V_{N}\left(z_{1}, \ldots, z_{N}\right)$, which are solutions of the original system (5.10). Let $\mathscr{F}_{(\lambda, a)}^{V_{1} \ldots V_{N}}\left(z_{1}, \ldots, z_{N}\right)$ be a basis for solutions to (5.10), such that

$$
\mathscr{F}_{(\lambda, a)}^{V_{1} \ldots V_{N}}\left(z_{1}, \ldots, z_{N}\right)=\prod_{i<j} G_{i j}\left(\frac{z_{i}}{z_{j}}\right) F_{(\lambda, a)}^{V_{1} \ldots V_{N}}\left(z_{1}, \ldots, z_{N}\right)
$$

Then the asymptotic behavior of $G_{i j}\left(\frac{z_{i}}{z_{j}}\right)$ and $F_{(\lambda, a)}^{V_{1} \ldots V_{N}}\left(z_{1}, \ldots, z_{N}\right)$ at $\left|z_{1}\right| \gg\left|z_{2}\right| \gg$ $\cdots \gg\left|z_{N}\right|$ given by (6.5), (6.8), and (6.9) implies

$$
\widetilde{F}_{(\lambda, a)}^{V_{1} \ldots V_{N}}\left(z_{1}, \ldots, z_{N}\right) \in \prod_{i=1}^{N} z_{i}^{h\left(\lambda_{\imath-1}\right)-h\left(\lambda_{i}\right)} \mathbf{C}\left(\left(z_{1}, \frac{z_{2}}{z_{1}}, \ldots, \frac{z_{N}}{z_{N-1}}\right)\right)
$$

and

$$
\mathscr{F}_{(\lambda, a)}^{V_{1} \ldots V_{N}}\left(z_{1}, \ldots, z_{N}\right) \rightarrow \prod_{i=1}^{N} z_{i}^{h\left(\lambda_{i-1}\right)-h\left(\lambda_{2}\right)} \eta_{\lambda_{0} \lambda_{1} \ldots \lambda_{N}}^{V_{1} \ldots V_{N}}\left(a_{1}, \ldots, a_{N}\right)
$$


Moreover, these functions can be analytically continued to any region $\mathscr{C}_{w}$ and

$$
\begin{aligned}
R_{s_{i}} \mathscr{F}_{(\lambda, a)}^{V_{\tau(1)} \ldots V_{\tau(N)}}\left(z_{1}, \ldots, z_{N}\right) \prod_{i=1}^{N} z_{i}^{-h\left(\lambda_{i-1}\right)+h\left(\lambda_{i}\right)} \\
=\sum_{\left(\lambda^{\prime}, a^{\prime}\right)} \mathscr{F}_{\left(\lambda^{\prime}, a^{\prime}\right)}^{V_{\tau(1)} \ldots V_{\tau(i+1)} V_{\tau(i)} \ldots V_{\tau(N)}}\left(z_{1}, \ldots, z_{i+1}, z_{i}, \ldots, z_{N}\right) \\
\quad \times \prod_{i=1}^{N} z_{i}^{-h\left(\lambda_{i+1}^{\prime}\right)+h\left(\lambda_{\imath}^{\prime}\right)}\left(S_{i}^{\tau}\left(\frac{z_{i}}{z_{i+1}}\right)\right)_{(\lambda, a)}^{\left(\lambda^{\prime}, a^{\prime}\right)} .
\end{aligned}
$$

Here $\left(S_{i}^{\tau}(z)\right)_{(\lambda, a)}^{\left(\lambda^{\prime}, a^{\prime}\right)}$ are matrix elements of a linear map

$$
S_{i}^{\tau}(z): \mathscr{H}_{\lambda_{0} \lambda_{N}}^{V_{\tau(1) \cdots} V_{\tau(N)}} \rightarrow \mathscr{H}_{\lambda_{0} \lambda_{N}}^{V_{\tau(1) \cdots V_{\tau(i+1)}} V_{\tau(i) \cdots V_{\tau(N)}}}
$$

and

$$
S_{i}^{\tau}(z)=K_{\tau(i) \tau(i+1)}(z) C_{i}^{\tau}(z)
$$

where $C_{i}^{\tau}(z)$ is given by (6.33) and $K_{i j}(z)$ is a "gauged out" connection matrix for the system (6.2). Since $R^{V_{\imath} V_{j}}(0)=\bar{R}^{V_{\imath} V_{j}}$, and, by definition, $\tilde{R}^{V_{i} V_{j}}(z) v_{0} \otimes w_{0}=w_{0} \otimes v_{0}$ ( $v_{0}$ and $w_{0}$ are $U_{q}(\mathfrak{g})$ highest weight vectors in $V_{i}$ and $V_{j}$, respectively) we have $\tilde{f}_{V_{i} V_{j}}(0)=q^{\left(\mu_{i} \mu_{\jmath}\right)}$, where $\mu_{i}$ is a $U_{q}(\mathfrak{g})$ highest weight of $V_{i}$ and $\mu_{j}$ is a highest weight of $V_{j}$. Then, using the unitarity (4.20) of $R^{V_{i} V_{j}}(z)$ we obtain two solutions for (6.2) given by power series at $z=0$ and $z=\infty$, respectively:

$$
\begin{gathered}
G_{i j}(z)_{0}=z^{-\frac{\left(\mu_{i} \mu_{j}\right)}{2(k+g)}} \prod_{n \geq 1} \tilde{f}_{V_{\imath} V_{\jmath}}^{0}\left(z p^{n}\right)^{-1}, \\
G_{i j}(z)_{\infty}=z^{-\frac{\left(\mu_{\imath} \mu_{\jmath}\right)}{2(k+g)}} \prod_{n \geq 0} \tilde{f}_{V_{\imath} V_{j}}^{0}\left(z^{-1} p^{n}\right)^{-1}
\end{gathered}
$$

where $\tilde{f}_{V W}^{0}(z)=q^{-\left(\mu_{i} \mu_{j}\right)} \tilde{f}_{V W}(z)$.

Therefore,

$$
K_{i j}(z)=\frac{G_{i j}(z)_{0}}{G_{i j}(z)_{\infty}} z^{\frac{\left(\mu_{\imath} \mu_{j}\right)}{k+g}}=\prod_{n \geq 0} \frac{\tilde{f}_{V_{i} V_{j}}^{0}\left(z^{-1} p^{n}\right)}{\tilde{f}_{V_{\imath} V_{j}}^{0}\left(z p^{n+1}\right)} .
$$

Let us identify now solutions to the $q$-analogue of the Knizhnik-Zamolodchikov equation with matrix elements of intertwining operators.

Theorem 6.5. Let $\mathscr{F}_{(\lambda, a)}^{V_{1} \ldots V_{N}}\left(z_{1}, \ldots, z_{N}\right)$ be the basis (6.34) for $U_{q}(\mathfrak{g})$-invariant solutions to the system (5.10), then

$$
\mathscr{F}_{(\lambda, a)}^{V_{1} \ldots V_{N}}\left(z_{1}, \ldots, z_{N}\right)=\sum_{i}\left\langle\cdot, \Phi_{1}^{V_{1}}\left(z_{1} \mid a_{1}\right)_{\lambda_{1}}^{\lambda_{0}} \ldots \Phi_{N}^{V_{N}}\left(z_{N} \mid a_{N}\right)_{\lambda N}^{\lambda_{N-1}} \cdot\right\rangle
$$

where $\Phi^{V}(z \mid a)_{\lambda}^{\mu}$ is a $U_{q}(\hat{\mathfrak{g}})$-intertwining operator corresponding to $a \in H_{\mu \lambda}^{V}$, and the equality holds over $\prod_{i=1}^{N} z_{i}^{h\left(\lambda_{i-1}\right)-h\left(\lambda_{i}\right)} \mathbf{C}\left(\left(z_{1}, \frac{z_{2}}{z_{1}}, \ldots, \frac{z_{N}}{z_{N_{1}}}\right)\right)$. 
Proof. By definition left and right sides of (6.39) satisfy (5.10). Both of them have the same asymptotics at $\left|z_{1} 0 \gg\right| z_{2}|\gg \cdots \gg| z_{N} \mid$. Therefore they. coincide on $\prod_{i=1}^{N} z_{i}^{h\left(\lambda_{i-1}\right)-h\left(\lambda_{2}\right)} \cdot \mathbf{C}\left(\left(z_{1}, \frac{z_{2}}{z_{1}}, \ldots, \frac{z_{N}}{z_{N-1}}\right)\right)$.

Since we have analytic continuation of the solution $\mathscr{F}_{(\lambda, a)}^{V_{1} \ldots V_{N}}\left(z_{1}, \ldots, z_{N}\right)$ to other regions, this theorem also describes the analytic continuation of products of vertex operators. In fact, all matrix coefficients of the composition

$$
\left\langle v^{\prime}, \Phi_{1}^{V_{1}}\left(z_{1}\right)_{\lambda_{1}}^{\lambda_{0}} \Phi_{2}^{V_{2}}\left(z_{2}\right)_{\lambda_{2}}^{\lambda_{1}} v\right\rangle, \quad v \in V_{\lambda_{2}, k}, v^{\prime} \in V_{\lambda_{0}, k}^{*}
$$

can be extended to meromorphic functions defined for all nonzero complex values of $z_{1}, z_{2}$. Thus we can define an operator $\mathscr{O}\left(\Phi_{1}^{V_{1}}\left(z_{1}\right) \Phi_{2}^{V_{2}}\left(z_{2}\right)\right)$ defined by its matrix coefficients.

Consider the following intertwining operators:

$$
\begin{aligned}
& \Phi_{1}^{V_{1}}\left(z_{1}\right)_{\lambda_{1}}^{\lambda_{0}} \Phi_{2}^{V_{2}}\left(z_{2}\right)_{\lambda_{2}}^{\lambda_{1}}: V_{\lambda_{2}, k} \\
& \rightarrow V_{\lambda_{0}, k} \otimes V_{1}\left(z_{1}\right) \otimes V_{2}\left(z_{2}\right) \otimes z_{1}^{h\left(\lambda_{0}\right)-h\left(\lambda_{1}\right)} z_{2}^{h\left(\lambda_{1}\right)-h\left(\lambda_{2}\right)} \cdot \mathbf{C}\left(\left(z_{1}, \frac{z_{2}}{z_{1}}\right)\right), \\
& P^{V_{1} V_{2}} R^{V_{1} V_{2}}\left(\frac{z_{1}}{z_{2}}\right) \Phi_{2}^{V_{2}}\left(z_{2}\right)_{\lambda_{1}^{\prime}}^{\lambda_{0}} \Phi_{1}^{V_{1}}\left(z_{1}\right)_{\lambda_{2}}^{\lambda_{1}^{\prime}}: V_{\lambda_{2}, k} \\
& \quad \rightarrow V_{\lambda_{0}, k} \otimes V_{1}\left(z_{1}\right) \otimes V_{2}\left(z_{2}\right) \otimes z_{1}^{h\left(\lambda_{0}\right)-h\left(\lambda_{1}^{\prime}\right)} z_{2}^{h\left(\lambda_{1}^{\prime}\right)-h\left(\lambda_{2}\right)} \cdot \mathbf{C}\left(\left(z_{2}, \frac{z_{1}}{z_{2}}\right)\right) .
\end{aligned}
$$

For analytical continuation of these operators, we have the following.

Theorem 6.6. Intertwining operators satisfy the following exchange algebra:

$$
\begin{aligned}
P R^{V_{1} V_{2}}\left(\frac{z_{1}}{z_{2}}\right) & \mathscr{Q}\left(\Phi_{1}^{V_{1}}\left(z_{1} \mid a_{1}\right)_{\lambda_{1}}^{\lambda_{0}} \Phi_{2}^{V_{2}}\left(z_{2} \mid a_{2}\right)_{\lambda_{2}}^{\lambda_{1}}\right) \\
= & \sum_{\substack{a_{1}^{\prime} a_{2}^{\prime} \\
\lambda_{1}^{\prime}}} \odot\left(\Phi_{2}^{V_{2}}\left(z_{2} \mid a_{2}\right)_{\lambda_{1}}^{\lambda_{0}} \Phi_{1}^{V_{1}}\left(z_{1} \mid a_{1}\right)_{\lambda_{2}}^{\lambda_{1}}\right) S^{V_{1} V_{2}}\left[\begin{array}{ccc}
a_{1}^{\prime} & \lambda_{0} & a_{1} \\
\lambda_{1}^{\prime} & & \lambda_{1} \\
a_{2}^{\prime} & \lambda_{2} & a_{2}
\end{array}\right]\left(\frac{z_{1}}{z_{2}}\right)
\end{aligned}
$$

where $S^{V_{1} V_{2}}$ is given by (6.37) and both $R^{V_{1} V_{2}}(z)$ and $S^{V_{1} V_{2}}(z)$ are unitary, crossingsymmetrical solutions of the Yang-Baxter relation (in vertex and IRF formulation, respectively).

Another quantization of $U(\hat{\mathfrak{g}})$ is the full Yangian $\hat{Y}(\mathfrak{g})$. The Yangian $Y(\mathfrak{g})$ is an infinite dimensional Hopf algebra described in [Dr1] which is a deformation of the universal enveloping algebra of the parabolic Lie subalgebra $\hat{\mathfrak{g}}_{+} \hookrightarrow \hat{\mathfrak{g}}$. We call the full Yangian corresponding deformation of $U(\hat{\mathfrak{g}})$. The algebra $\hat{Y}(\mathfrak{g})$ can be naturally considered as an appropriate quotient of the double $\mathscr{D}(Y(\mathfrak{g}))$. Such a description of $\hat{Y}(\mathfrak{g})$ was given by Smirnov [Sm1].

Similarly as we did it for $U_{q}(\hat{\mathfrak{g}})$ one can construct for $\hat{Y}(\mathfrak{g})$ elements $\mathscr{B}^{ \pm, V}(x)$ which will play the role of currents $J(x)$ in a parabolic polarization. All relations between $\mathscr{L}^{ \pm, V}(x)$ and intertwiners one can obtain in the limit $z=q^{x}, q \rightarrow 1$ from our formulas. In particular, this limit in Eq. (6.40) gives us a difference analogue of Knizhnik-Zamolodchikov equations for $\hat{Y}(\mathfrak{g})$. In the same way, one can define 
connection matrices for $\hat{Y}(\mathfrak{g})$. But now they will be trigonometric functions of $x$. Since the intertwiner of $\hat{Y}(\mathfrak{g})$ can be obtained from $U_{q}(\hat{\mathfrak{g}})$-intertwiners, we expect that the following conjecture is true.

Conjecture 2. Let $q^{\prime}=\exp \left(\frac{2 \pi i}{k+g}\right)$ and $R^{V_{1} V_{2}}(z)$ is a finite-dimensional $R$-matrix for $U_{q^{\prime}}(\hat{\mathfrak{g}})$ and $\eta_{\lambda_{0} \lambda_{2}}^{V_{1} V_{2}}$ is the isomorphism (6.0). Then

$$
\begin{aligned}
& \left(1 \otimes \check{R}^{V_{1} V_{2}}\left(\exp \left(\frac{2 \pi i x}{k+g}\right)\right) \otimes 1\right) \eta_{\lambda_{0} \lambda_{1} \lambda_{2}}^{V_{1} V_{2}} \\
& \quad=\lim _{q \rightarrow 1} \sum_{\lambda_{1}^{\prime}} \eta_{\lambda_{0} \lambda_{1} \lambda_{2}}^{V_{1} V_{2}} \cdot S^{V_{1} V_{2}}\left[\begin{array}{ccc}
\lambda_{1}^{\prime} & & \lambda_{1} \\
& \lambda_{2} &
\end{array}\right]\left(q^{x}\right),
\end{aligned}
$$

where $S^{V_{1} V_{2}}\left[\begin{array}{ccc} & \lambda_{0} & \\ \lambda_{1}^{\prime} & & \lambda_{1} \\ & \lambda_{2} & \end{array}\right]$ is a linear map $\mathscr{H}_{\lambda_{0} \lambda_{1}}^{V_{1}} \otimes \mathscr{H}_{\lambda_{1} \lambda_{2}}^{V_{2}} \rightarrow \mathscr{H}_{\lambda_{0} \lambda_{1}^{\prime}}^{V_{2}} \otimes \mathscr{H}_{\lambda_{1} \lambda_{2}}^{V_{1}}$ determined by (6.37).

This conjecture can be thought as a generalization of Theorem 3 on the equivalence of the tensor categories $\operatorname{Mon}_{k}(\hat{\mathfrak{g}})$ and $\operatorname{Rep}_{q}(\mathfrak{g})$. In fact, the Yang-Baxter relation (6.22) and the unitarity condition (6.23) generalize the alternative axioms (2.51), (2.52) of pre-tensor category defined in Sect. 2 . The new ingredient in our case is the analytic dependence of the connection matrices on the complex parameter. This suggests the notion of analytic pre-tensor category, which differs from the pre-tensor category by assigning now to any three objects $X, Y, Z$ a family of meromorphic maps

$$
\beta_{X, Y, Z}(x):(X \otimes Y) \otimes Z \rightarrow(X \otimes Z) \otimes Y,
$$

where $x \in \mathbf{C}, \mathbf{C} / \mathbf{Z}$ or $\mathbf{C} / \mathbf{Z} \otimes \tau \mathbf{Z}$, satisfying the star-triangle relation and the unitarity. The three cases correspond to the rational, trigonometric and elliptic solutions, respectively. In the trigonometric case setting

$$
\beta_{X, Y, Z}^{ \pm}=\lim _{x \rightarrow \pm \infty} \beta_{X, Y, Z}(x)
$$

we recover a pre-tensor category.

The above considerations point to the problem of defining a natural notion of analytic tensor category, which should include an appropriate generalzation of the pentagon identity. For our example, this translates into the problem of finding a quantum analogue of the operator product expansion in conformal field theory. Here the positions of poles and zeros of operators $R^{V W}(z)$ should play an important role. Additional structures of solutions of star-triangle relations (and/or Yang-Baxter operators) such as the crossing symmetry (4.17), (4.18) should provide the analytic tensor category with the additional structures generalizing the rigidity in the case of tensor category defined in Sect. 3.

\section{Examples of Solutions of the Difference Equations for $U_{q}\left(\widehat{\mathfrak{s l}_{2}}\right)$ and the Theory of Basic Hypergeometric Functions}

Solutions of the Knizhnik-Zamolodchikov equations are given by the vast generalization of the hypergeometric functions associated to any simple Lie algebra. These 
solutions have a very rich structure. In particular, the monodromies of the solutions are closely related to the trigonometric solutions of the Yang-Baxter equation and manifest hidden symmetries realized by quantum groups. It is natural to expect that the solutions of the holonomic difference equations associated to the quantum affine algebras are certain $q$-deformations of the generalized hypergeometric functions and that connection matrices for these $q$-hypergeometric functions are elliptic solutions of the Yang-Baxter equations, which is fully confirmed by the results of Sect 6. It is an important open problem to find an algebraic structure of hidden symmetries of our difference equations, which should be an elliptic version of quantum groups.

In this section we will consider the simplest nontrivial example of our equations associated to quantum affine algebra $U_{q}(\widehat{\mathfrak{s l}}(2))$. However, already this example allows us to grasp the essence of one of the classical chapters of mathematics, the theory of basic hypergeometric series.

A $q$-analogue of the hypergeometric series known as the basic hypergeometric series was introduced in the middle of the last century [H1, H2]:

$$
F_{q}(a, b, c ; z)=\sum_{n=0}^{\infty} \frac{\left(q^{a}\right)_{n}\left(q^{b}\right)_{n}}{[n] !\left(q^{c}\right)_{n}} z^{n}
$$

(the standard notation is ${ }_{2} \phi_{1}\left(q^{a}, q^{b} ; q^{c} ; q^{z}\right)$ ), where we denote

$$
(a)_{n}=(1-a)(1-a q) \ldots\left(1-a q^{n-1}\right) \text {. }
$$

The basic hypergeometric function $F_{q}(a, b, c ; z)$ satisfies the difference equation

$$
(z[\delta+a][\delta+b]-[\delta][\delta+c-1]) F_{q}(a, b, c, z)=0,
$$

where

$$
[\delta+a]=\frac{1-q^{\delta+a}}{1-q}=\frac{\left(q^{\delta+a}\right)_{0}}{(q)_{0}}
$$

and

$$
q^{\delta} f(z)=f(q z)
$$

Omission of the square brackets in (7.3) yields the ordinary differential equation for the classical hypergeometric function with $\delta=z \frac{d}{d z}$. There also exist $q$-analogues of various special functions. For example, the $q$-analogue of the gamma function $\Gamma_{q}$ :

$$
\Gamma_{q}(a)=\frac{(q)_{\infty}}{\left(q^{a}\right)_{\infty}}(1-q)^{1-a}
$$

and the power function

$$
(1-z)_{q}^{2 m}=\frac{\left(q^{-m} z\right)_{\infty}}{\left(q^{m} z\right)_{\infty}}
$$

When $q \rightarrow 1$, these functions tend to their classical counterparts. Most of the identities for special functions have their $q$-analogues. However, in some cases, new phenomena appear. 
One can also introduce $q$-analogues of differentiation and integration

$$
\begin{gathered}
\frac{d_{q} f}{d_{q} t}(t)=\frac{1}{t} \cdot \frac{f(t)-f(q t)}{1-q}, \\
\int_{0}^{c} f(t) d_{q} t=c(1-q) \sum_{n \geq 0} f\left(c q^{n}\right) q^{n}, \\
\int_{c}^{\infty} f(t) d_{q} t=c(1-q) \sum_{n<0} f\left(c q^{n}\right) q^{n} .
\end{gathered}
$$

Again, when $q \rightarrow 1$, these operations become the usual differentiation and integration.

As in the classical case, the basic hypergeometric function has an integral presentation, but now in terms of Jackson integral [Ja],

$$
F_{q}(a, b, c, z)=\frac{\Gamma_{q}(c)}{\Gamma_{q}(a) \Gamma_{q}(c-a)} \int_{0}^{1} t^{a-1} \frac{(q t)_{\infty}\left(q^{b} z t\right)_{\infty}}{\left(q^{c-a} z\right)_{\infty}(z t)_{\infty}} d_{q} t
$$

The main property of the basic hypergeometric series that we will need is the connection formulas between the solutions with prescribed asymptotic behavior at $z \rightarrow 0$ and $z \rightarrow \infty$. In accordance with the general theory of linear $q$-difference equations, the connection coefficients should involve the Jacobi elliptic theta functions. In fact, the following connection formula for the basic hypergeometric series is valid:

$$
\begin{aligned}
F_{q}(a, b, c ; z)= & \frac{\Gamma_{q}(c) \Gamma_{q}(b-a)}{\Gamma_{q}(b) \Gamma_{q}(c-a)} \frac{\Theta\left(q^{a} z, q\right)}{\Theta(z, q)} \\
& \times F_{q}\left(a, a-c+1, a-b+1, q^{c+1-a-b} z^{-1}\right) \\
& +\frac{\Gamma_{q}(c) \Gamma_{q}(a-b)}{\Gamma_{q}(a) \Gamma_{q}(c-b)} \frac{\Theta\left(q^{b} z, q\right)}{\Theta(z, q)} \\
& \times F_{q}\left(b, b-c+1, b-a+1, q^{c+1-a-b} z^{-1}\right) .
\end{aligned}
$$

When $q \rightarrow 1$, the ratios of the theta-functions in (7.14) tend to $(-z)^{-a}$ and $(-z)^{-b}$ respectively, $\Gamma_{q}$ and $F_{q}$ tend to their classical counterparts and we get the connection formula for the classical hypergeometric function.

Remark 7.1. Basic hypergeometric series appear as the matrix coefficients of the quantum group associated to $S U(2)$, [SoV, Koo, MMNNU]. However, their most important properties such as integral presentation and connection formulas can only be revealed in the representation theory of quantum affine algebras.

Let us consider a simplest nontrivial example of holonomic difference equations associated to quantum affine algebras, where the above formulas find the conceptual meaning. Let $\mathfrak{g}=\mathfrak{s l}(2)$, and let $V$ be an irreducible representation of $U_{q}(\mathfrak{g})$ of dimension $M+1$, i.e. has highest weight $M \alpha / 2$, where $\alpha$ is the positive root of $\mathfrak{s l}$. One, may choose the basis $v_{0}, v_{1}, \ldots, v_{M}$ so that

$$
\pi\left(e_{1}\right) v_{n}=[n] v_{n-1}, \quad \pi\left(f_{1}\right) v_{n}=[M-n] v_{n+1}, \quad \pi\left(k_{1}\right) v_{n}=q^{M-2 n} v_{n}
$$

This representation can be extended to a representation of $U_{q}(\hat{\mathfrak{g}})$ depending on a nonzero parameter $z$,

$$
\pi\left(e_{0}\right)=z \pi\left(f_{1}\right), \quad \pi\left(f_{0}\right)=z^{-1} \pi\left(e_{1}\right), \quad \pi\left(k_{0}\right)=\pi\left(k_{1}\right)^{-1} .
$$


Let us show that solutions to the system (6.25) can be naturally described in terms of $q$-hypergeometric functions. Consider the simplest case when $V_{1} \cong V_{2} \cong V_{\frac{\alpha}{2}}$ are two-dimensional $U_{q}\left(\widehat{\mathfrak{s l}}_{2}\right)$ modules. Also in this case, we can directly use system (6.25) and we will use the isomorphism

$$
\operatorname{Inv}_{U_{q}\left(\mathfrak{s i}_{2}\right)}\left(V_{\lambda_{0}} \otimes V_{1} \otimes V_{2} \otimes\left(V_{\lambda_{2}}\right)^{*}\right) \stackrel{\sim}{\rightarrow}\left(V_{1} \otimes V_{2}\right)\left\{\lambda_{0}-\lambda_{2}\right\}
$$

which holds in this particular case and we will reduce (6.25) to a simpler system.

Assume that $\lambda_{0}=M_{\frac{\alpha}{2}}, \lambda_{2}=\lambda_{0} \pm \alpha$. Then, choosing in (6.1), $\alpha_{i}=1$, we have the following basis in the left-hand side of (7.15):

$$
\begin{aligned}
\eta_{\lambda_{0}, \lambda_{0}+\frac{\alpha}{2}, \lambda_{0}+\alpha} & =v_{0} \otimes v_{0} \otimes v_{0} \otimes v_{0}^{\prime}+\ldots \\
\eta_{\lambda_{0}, \lambda_{0}+\frac{\alpha}{2}, \lambda_{0}} & =v_{0} \otimes\left(v_{0} \otimes v_{1}-\frac{q^{-M-1}}{[M+1]} v_{1} \otimes v_{0}\right) \otimes v_{0}^{\prime}+\ldots \\
\eta_{\lambda_{0}, \lambda_{0}-\frac{\alpha}{2}, \lambda_{0}} & =v_{0} \otimes v_{1} \otimes v_{0} \otimes v_{0}^{\prime}+\ldots \\
\eta_{\lambda_{0}, \lambda_{0}-\frac{\alpha}{2}, \lambda_{0}-\alpha} & =v_{0} \otimes v_{1} \otimes v_{1} \otimes v_{0}^{\prime}+\ldots
\end{aligned}
$$

Here $v_{0}^{\prime}$ is the lowest weight vector in $\left(V_{\lambda_{2}}\right)^{*}$. In (7.16), we only write terms containing the highest weight vector in $V_{\lambda_{0}}$ and the lowest weight vector in $V_{\lambda_{2}}$.

The isomorphism (7.15) is given explicitly by the map

$$
\eta_{\lambda_{0}, \lambda_{1}, \lambda_{2}} \mapsto \xi_{\lambda_{0}, \lambda_{1}, \lambda_{2}}=\left\langle v_{0}^{\prime} \otimes \mathrm{id} \otimes \mathrm{id} \otimes v_{0}, \eta_{\lambda_{0} \lambda_{1} \lambda_{2}}\right\rangle
$$

Let us return to the system (6.25). Let $\varphi_{(\lambda, a)}(z)$ be a basis to solutions of this system. The following proposition is a simple corollary of (4.42) and (4.43).

Proposition 7.1. Vectors $\psi_{(\lambda, a)}(z)=\left\langle v_{0}^{\prime} \otimes \mathrm{id} \otimes \mathrm{id} \otimes v_{0}, \varphi_{(\lambda, a)}(z)\right\rangle$ are solutions of the system

$$
T \psi_{(\lambda, a)}(z)=q^{\frac{C\left(\lambda_{0}\right)-C\left(\lambda_{2}\right)}{2}}\left(q^{\lambda}\right)_{2} \tilde{R}_{12}(z) \psi_{(\lambda, a)}(z)
$$

where $\lambda=\lambda_{0}+\lambda_{2}+2 \varrho$, and $\psi_{(\lambda, a)}(z) \in\left(V_{1} \otimes V_{2}\right)\left\{\lambda_{2}-\lambda_{0}\right\}$.

In our case, since we have the isomorphism (7.15), there is a one-to-one correspondence between solutions to (6.25) and (7.17), and we can study simpler system (7.17) in order to obtain solutions to (6.25).

By definition of $\tilde{R}(z)$, we have:

$$
\tilde{R}(z) v_{0} \otimes v_{0}=v_{0} \otimes v_{0}, \quad \tilde{R}(z) v_{1} \otimes v_{1}=v_{1} \otimes v_{1} .
$$

Therefore, if $\lambda_{2}=\lambda_{0} \pm \alpha$, we have the following solutions of (7.17):

$$
\begin{aligned}
& \psi_{\lambda_{0}, \lambda_{0}+\frac{\alpha}{2}, \lambda_{0}+\alpha}(z)=z^{\frac{h\left(\lambda_{2}\right)-h\left(\lambda_{0}\right)}{2}-\frac{M+2}{2(k+g)}} v_{0} \otimes v_{0}, \\
& \psi_{\lambda_{0}, \lambda_{0}-\frac{\alpha}{2}, \lambda_{0}-\alpha}(z)=z^{\frac{h\left(\lambda_{2}\right)-h\left(\lambda_{0}\right)}{2}-\frac{M+2}{2(k+g)}} v_{1} \otimes v_{1} .
\end{aligned}
$$

The case when $\lambda_{2}=\lambda_{0}$ is a particular case of the following system. Let in (7.17) $V_{1} \cong V_{\frac{M_{1} \alpha}{2}}, V_{2} \cong V_{\frac{M_{2} \alpha}{2}}$, and $\lambda_{0}=M_{0} \frac{\alpha}{2}, \lambda_{2}=\left(M_{0}+M_{1}+M_{2}-2\right) \frac{\alpha}{2}$. It is not difficult to find solutions explicitly in this case also. From the definition of $\tilde{R}_{12}(z)$ as 
an intertwining operator we compute its action in the basis $v_{1} \otimes v_{m}$ (see $[\mathrm{KR}]$ for general matrix elements):

$$
\begin{aligned}
& \tilde{R}(z) v_{0} \otimes v_{1}=\frac{z q^{M_{1}}-q^{M_{2}}}{z-q^{M_{1}+M_{2}}} v_{0} \otimes v_{1}+\frac{z\left(q^{2 M_{1}}-1\right)}{q^{M_{1}+M_{2}}-z} v_{1} \otimes v_{0}, \\
& \tilde{R}(z) v_{1} \otimes v_{0}=\frac{\left(q^{2 M_{2}}-1\right)}{q^{M_{1}+M_{2}}-z} v_{0} \otimes v_{1}+\frac{z q^{M_{2}}-q^{M_{1}}}{z-q^{M_{1}+M_{2}}} v_{1} \otimes v_{0} .
\end{aligned}
$$

Solutions of the system (7.17) in the subspace $\left(V_{\mu_{1}} \otimes V_{\mu_{2}}\right)\left\{\mu_{1}+\mu_{2}-\alpha\right\}$ can be given in terms of basic hypergeometric functions which we will write in the integral form:

$$
\psi\left(\frac{z_{1}}{z_{2}}\right)=\psi_{1}\left(z_{1}, z_{2}\right) v_{0} \otimes v_{1}+\psi_{2}\left(z_{1}, z_{2}\right) v_{1} \otimes v_{0}
$$

Then we have

$$
\psi_{j}^{i^{\prime}}\left(z_{1}, z_{2}\right)=\left(\frac{z_{1}}{z_{2}}\right)^{\frac{h\left(\lambda_{2}\right)-h\left(\lambda_{0}\right)}{2}-\frac{\left(\lambda, \mu_{1}-\alpha\right)}{2(k+g)}} f_{j}^{i^{\prime}}\left(z_{1}, z_{2}\right)
$$

and

$$
\left\{\begin{aligned}
f_{1}^{i^{\prime}\left(z_{1}, z_{2}\right)=} & p^{m_{1}-m} \int_{0}^{z_{i}^{\prime}}\left(\frac{t}{z_{1}}\right)^{m_{1}-m-1}\left(\frac{t}{z_{2}}\right)^{m_{2}-m} \\
& \times \partial_{p}^{+}\left(1-\frac{t}{z_{1}}\right)_{p}^{-2 m_{1}}\left(1-\frac{t}{z_{2}}\right)_{p}^{-2 m_{2}} d_{p} t \\
f_{2}^{i^{\prime}}\left(z_{1}, z_{2}\right)= & p^{m} \int_{0}^{z_{i}^{\prime}}\left(\frac{t}{z_{1}}\right)^{m_{1}-m}\left(\frac{t}{z_{2}}\right)^{m_{2}-m-1}\left(1-\frac{t}{z_{1}}\right)_{p}^{-2 m_{1}} \\
& \times \partial_{p}^{-}\left(1-\frac{t}{z_{2}}\right)_{p}^{-2 m_{2}} d p t \\
& \times \partial_{p}^{+}\left(1-\frac{z_{1}}{t}\right)_{p}^{-2 m_{1}}\left(1-\frac{z_{2}}{t}\right)_{p}^{-2 m_{2}} d_{p} t \\
f_{1}^{i^{\prime \prime}}\left(z_{1}, z_{2}\right)= & p^{-\frac{1}{2}-m} \int_{z_{i}^{\prime \prime}}^{\infty}\left(\frac{z_{1}}{t}\right)^{m_{1}+m}\left(\frac{z_{2}}{t}\right)^{m_{2}+m} \\
& \times \partial_{p}^{-}\left(1-\frac{z_{2}}{t}\right)_{p}^{-2 m_{2}} d_{p} t, \\
f_{2}^{i^{\prime \prime}\left(z_{1}, z_{2}\right)=} & p^{m_{2}+m}\left(\frac{z_{1}}{t}\right)^{m_{1}+m}\left(\frac{z_{2}}{t}\right)^{m_{2}+m}\left(1-\frac{z_{1}}{t}\right)_{p}^{-2 m_{1}}
\end{aligned}\right.
$$

where we denoted $\partial_{p}^{ \pm}=\frac{d_{p^{ \pm 1}}}{d_{p^{ \pm 1}} t}$ and

$$
z_{1}^{\prime}=z_{1} p^{-m_{1}}, \quad z_{2}^{\prime}=z_{2} p^{-m_{2}}, \quad z_{1}^{\prime \prime}=z_{1} p^{m_{1}}, \quad z_{2}^{\prime \prime}=z_{2} p^{m_{2}} \text {. }
$$


Here $m_{i}=M_{i} / 2(k+g), m=\left(M_{0}+M_{1}+M_{2}-2\right) / 4(k+g)$. The expression of our four solutions by means of the basic hypergeometric series immediately yields the correct asymptotic behavior, thus $f^{2^{\prime}}$ and $f^{1^{\prime \prime}}$ are power series in $\frac{z_{2}}{z_{1}}$ while $f^{1^{\prime}}$ and $f^{2^{\prime \prime}}$ are power series in $\frac{z_{1}}{z_{2}}$. Let us denote for simplicity the components of the connection matrix by $B(z)_{j}^{i}, i, j=1$ or 2 , so that

$$
\left(\begin{array}{l}
f^{1^{\prime \prime}} \\
f^{2^{\prime}}
\end{array}\right)=\left(\begin{array}{ll}
B(z)_{1}^{1} & B(z)_{2}^{1} \\
B(z)_{1}^{2} & B(z)_{2}^{2}
\end{array}\right)\left(\begin{array}{l}
f^{1^{\prime}} \\
f^{2^{\prime \prime}}
\end{array}\right)
$$

In order to find $B(z)_{j}^{i}$, we can either use the connection formula for the basic hypergeometric functions (7.12) or deduce it directly using the method of Mimachi [M] from the Cauchy residue formula for the following meromorphic functions, which relate in each case three out of four Jackson integrals in (7.22) and (7.23). The final answer for $B(z)_{j}^{i}$ has the following form:

\section{Proposition 7.2.}

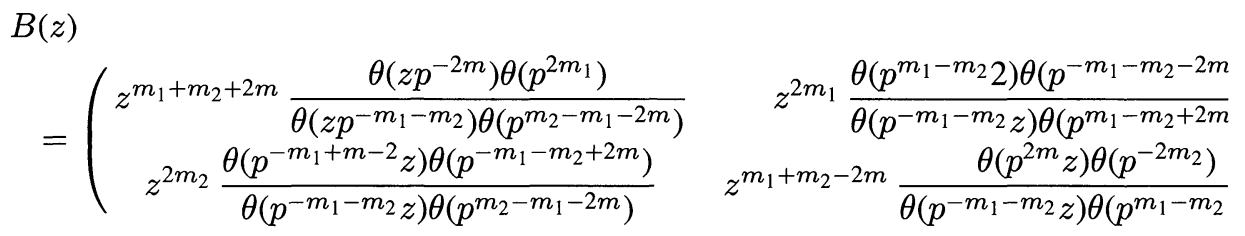

This concludes the description of the connection matrix for the quantum affine algebra $U_{1}(\widehat{\mathfrak{s} l}(2))$ for $\lambda_{0}=\mu_{1}+\mu_{2}+\lambda_{2}-\alpha$. The generalization to the case $\lambda_{0}=$ $\mu_{1}+\mu_{2}+\lambda_{2}-n \alpha$, where $n$ is an arbitrary positive integer is an interesting technical problem. It is clear that the solutions are given by multiple Jackson integrals, which are $q$-analogues of the explicit solutions of the Knizhnik-Zamolodchikov equations obtained in [SV1]. Their monodromies will be given by $(n+1) \times(n+1)$ matrices with entries expressed in terms of the products of elliptic theta functions as in (7.25). For a given $\mu_{1}$ and $\mu_{2},(7.25)$ give corresponding matrix elements elliptic solutions to the star-triangle relations, which in the $\mathfrak{s l}(2)$-case were computed explicitly in [DJKMO]. In fact, in the case when $n=1$ one can easily check the coincidence of our connection matrix (7.25) with the corresponding formulas in [B1, ABF, DJKMO].

Let us return to our case $M_{1}=M_{2}=1$. Solutions $\psi^{2^{\prime}}\left(z_{1}, z_{2}\right)$ and $\psi^{1^{\prime \prime}}\left(z_{1}, z_{2}\right)$ are given by power series in $\frac{z_{2}}{z_{1}}$ and it is easy to check that for $M_{1}=M_{2}=1$,

$$
\begin{aligned}
& \psi^{2^{\prime}}\left(z_{1}, z_{2}\right)=\psi_{\lambda_{0}, \lambda_{1}+\alpha, \lambda_{0}}\left(\frac{z_{1}}{z_{2}}\right), \\
& \psi^{1^{\prime \prime}}\left(z_{1}, z_{2}\right)=\psi_{\lambda_{0}, \lambda_{0}-\alpha, \lambda_{0}}\left(\frac{z_{1}}{z_{2}}\right) .
\end{aligned}
$$

Therefore, we can identify now matrix elements of (7.25) with matrix elements of elementary connection matrices $\tilde{C}_{i}^{\tau}(z)$ from Sect. 6 and therefore with solutions to the Yang-Baxter equation. Formulas (7.25) and (7.26) give the following ratios of theta 
functions. Let us set $q=\exp (-i \pi \tau), z=\exp (2 \pi i \tau u),[x]=\theta(\exp (2 \pi i \tau x))$. Then one has

\section{Corollary 7.1.}

$$
\begin{aligned}
& W^{11}\left[\begin{array}{ccc}
M-1 & M & M-1 \\
& M-2
\end{array}\right](z)=W^{11}\left[\begin{array}{ccc}
M+1 & M & \\
& M+2
\end{array}\right](z)=1, \\
& W^{11}\left[\begin{array}{ccc} 
& M & \\
M+1 & & M-1
\end{array}\right](z)=\frac{[u][M+2]}{[u+1][M+1]},
\end{aligned}
$$

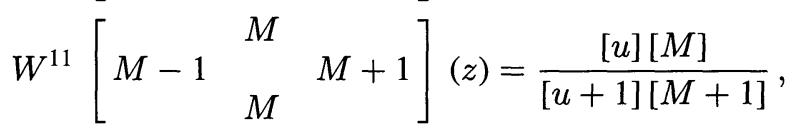

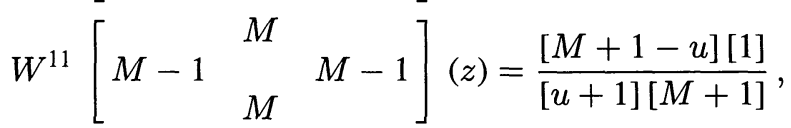

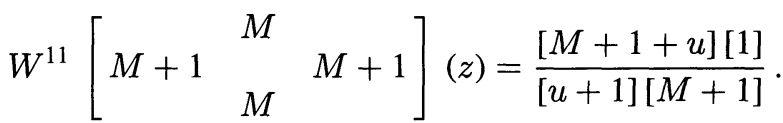

These formulas are exactly the solution for Eq. (6.22) which was found by Baxter, [B1] (see also [ABF, DJKMO]).

The exact coincidence of the formulas are manifest from the comparison with $(2.1 .4 \mathrm{a}, \mathrm{b}, \mathrm{c})$ in [DJKMO], provided that their solution is normalized by multiplication $\frac{[1]}{[u+1]}$ and the parameter $\xi=1$. Note that the star-triangle relations will be preserved under any shift of $M$, which explains the additional parameter $\xi$ in [DJKMO].

The description of the analogue of half-monodromy for the intertwining operators in terms of elliptic theta-functions indicates the presence of a new hidden symmetry. By comparison with the conformal field theory, where the exchange algebra reflects the presence of the trigonometric solution of the Yang-Baxter equation, our elementary example yields the elliptic solution of the Yang-Baxter equation. Moreover, the trigonometric solutions of the Yang-Baxter equations immediately bring us to the notion of the quantum group, which is the hidden symmetry of the conformal field theory. Thus we are forced to conjecture that the symmetry of the corresponding $q$ analogue is described by a new algebraic structure related to the elliptic solutions of the Yang-Baxter equation. In fact, a candidate for a new algebraic structure intimately related to the elliptic solutions of the Yang-Baxter equation, has been introduced in [Sk1, Sk2]. However, until recently it was not clear how this algebraic structure generalizes the Hopf algebra structure of quantum groups. We believe that the parallel between the trigonometric and elliptic solutions of the Yang-Baxter equations arising from the difference equations will shed new light to these previously formidable problems.

\section{Conclusion}

Results of this paper provide only the very first steps towards the understanding of a $q$-analogue of conformal field theory, of an elliptic generalization of the quantum group, the relations between them and the mathematical and physical implications of 
these new structures. However, already at this stage we can formulate a number of concrete problems and directions for future research.

The first problem discussed in Sect. 6 is an extension of the correspondence between the monodromies of the Knizhnik-Zamolodchikov equation and the representations of quantum algebras $U_{q}(\mathfrak{g})$ to the case of the difference and $q$-difference equations. We naturally expect that the corresponding connection matrices will be related to the trigonometric and elliptic solutions of the Yang-Baxter equation. Moreover, since we know that the trigonometric solutions can be interpreted as the intertwining operators for the finite dimensional representations of the quantum affine algebra $U_{q}(\hat{\mathfrak{g}})$ one can ask for a similar algebraic interpretation of the elliptic solutions; That is: what is the elliptic deformation of $U_{q}(\hat{\mathfrak{g}})$ and how it yields the connection matrices for the $q$-difference equations? Certainly an appropriate cohomological interpretation of the relation analogues to [SV2] could be of great interest.

The next problem is to understand the algebraic structure of a $q$-analogue of conformal field theory, in particular, a correct generalization of the operator product expansion or fusion. The solution of this problem should provide an explicit axiomatic definition of tensor categories with dependence on a complex parameter or, as we call them, analytic tensor categories.

As we explained in Sect. 6, the connection matrices for the $q$-difference equations provide elliptic solutions of the star-triangle relations for any type of the root system, thus bringing this large subfield of statistical mechanics into the realm of the representation theory. It can be fruitful for both fields to further extend the conceptual understanding of the integrable models of statistical mechanics. For example, an explanation of the coincidence of affine characters and local state probabilities [DJMO1] can now be more accessible than before. The similar remarks also apply to the massive integrable quantum field theory. As it is shown in [Sm1], the latter admits a striking reformulation in terms of axioms for form factors, which are directly related to our $q$-difference equations.

Besides the basic problems related to a correct understanding of a $q$-analogue of conformal field theory and its new symmetries, one can pose some further questions related to other known features of the undeformed case. We will briefly address the ones we consider most important.

One of the most subtle properties of quantum groups is its behavior when the parameter $q$ is equal to a root of unity. At these points the naive parallel with the classical theory breaks down and one encounters new arithmetric phenomena. In the case of quantum affine algebras and associated elliptic algebras, the role of two special parameters (namely the deformation parameter $q$ and the level $k$ in the case of the quantum affine algebras) can be even more significant. The arithmetic of the circle is now being replaced by the arithmetic of the elliptic curve. It is interesting to note that the values of $q$ which are roots of unity correspond to the cusps for the modular group $P S L(2, \mathbf{Z})$. The representation theory of quantum affine algebras may bring a new meaning to formulas of the arithmetic theory of elliptic curves.

The WZNW-model was first formulated [W1] as a certain Lagrangian theory and only later was translated in a pure algebraic language. In the case of its $q$-deformation, we can ask an opposite question, what is the geometric, i.e. Lagrangian, formulation of the algebraic theory that we described in Sects. 4 and 5. Certainly the answer should involve a certain example of noncommutative geometry. This could be a natural example for the general program of A. Connes.

Quantum algebras $U_{q}(\mathfrak{g})$ and WZNW conformal field theory have important topological applications to knot and 3-dimensional invariants [W2, RT1]. One can naturally 
ask about the topological implications of our $q$-deformed case. Since our connection matrices and the appropriate tensor categories depend on a parameter, one should try to associate with them a pure topological data. One can compare this situation to the passage from the conformal field theory to the topological field theory one dimension higher. Since in our case we have categories depending on a parameter, one should expect that a similar step will yield certain 2-categories, which in their turn are related to 2-knot invariants in $\mathbf{R}^{4}$ and eventually to the four-dimensional invariants.

Finally we would like to recall the analogy with the quantum mechanics, which is a quantization of classical mechanics, again admits a classical interpretation using functional integrals one (functional) dimension higher. One can expect that the representation theory of the quantum affine algebras can be obtained from the representation theory of double loop algebras like quantum groups appear as symmetries of conformal field theory.

Acknowledgements. We would like to thank H. Garland, D. Kazhdan, G. Moore and F. Smirnov for interesting discussions. We are grateful to MSRI for their hospitality during the January 1991 workshop "Strings in Mathematics \& Physics," where the main constructions and ideas of this work were invented. N. Reshetikhin would like to thank the Departments of Mathematics and Physics of Yale University for their hospitality during July 1991.

The main results of this work were presented in the conference "XXth International Congress on Differential Geometric Methods in Theoretical Physics" (New York, June 6-10, 1991), "Algebraic Groups and their Generalizations" (Penn State, AMS Summer Institute, July 8-26, 1991). We are pleased to thank all of those who participated in the organization of these meetings. The work of I. Frenkel was supported by Grant DMS-8906772 and by the Guggenheim Foundation Fellowship. The work of N. Reshetikhin was supported by NSF Grant DMS-9015821.

\section{References}

\section{Conformal Field Theory and Quantum Groups}

$[\mathrm{Ab}]$

[A]

$[\mathrm{BD}]$

[Ch]

$[\mathrm{DFa}]$

Abe, E.: Hopf algebras. Cambridge: Cambridge University Press 1980

Aomoto, K.: Gauss-Manin connection of integral of difference products. J. Math. Soc. Jpn 10, 191-208 (1987)

Belavin, A.A., Drinfeld, V.G.: Triangle equation and simple Lie algebras. Funct. Anal i ego Pril. 16, 1-29 (1982)

Belavin, A.A., Polyakov, A.N., Zamolodchikov, A.B.: Infinite conformal symmetries in two-dimensional quantum field theory. Nulc. Phys. B 241, 333-380 (1984)

Borcherds, R.E.: Vertex algebras, Kac-Moody algebras and the Monster. Proc. Natl. Acad. Sci. USA 83, 3068-3071 (1986)

Cherednik, I.: Integral solutions of trigonometric Knizhnik-Zamolodchikov equations and Kac-Moody algebras. Publ. RIMS, Kyoto Univ. 27, 727-744 (1991)

Dotsenko, Vl.S., Fateev, V.A.: Conformal algebra and multipoint correlation function in $2 D$ statistical models. Nulc. Phys. B 240 [FS12], 312 (1984); Nucl. Phys. B 251, 691 (1985)

Drinfeld, V.G.: On almost cocommutative Hopf algebras. Leningrad Math. J. 1, 321342 (1990)

Drinfeld, V.G.: Quasi-Hopf algebras. Leningrad Math. J. 1, 1419-1457 (1990)

Faddeev, L.D., Reshetikhin, N.Ya., Takhtajan, L.A.: Quantization of Lie groups and Lie algebras. Leningrad Math. J. 1, 193-226 (1990)

Fateev, V.A., Zamolodchikov, A.B.: Parafermionic $Z_{N}$-models. Sov. J. Nucl. Phys. 43, 637 (1986)

Friedan, D., Shenker, S., Qiu, Z.: Conformal invariance, unitarity and two-dimensional critical exponents. MSRI publications Vol. 3, pp.419-449. Berlin, Heidelberg, New York: Springer 1985

Frenkel, I.B., Huang, Y.-Z., Lepowsky, J.: On axiomatic approaches to vertex operator algebras and modules. Memoirs AMS (1992) 
[FLM] Frenkel, I.B., Lepowsky, J., Meurman, A.: Vertex operator algebras and the Monster. Boston: Academic Press 1988

[FZ] Frenkel, I.B., Zhu, Y.: Vertex operator algebras associated to representations of affine and Virasoro algebras. Duke Math. J. (1992)

[JS] Joeal, A., Street, R.: Braided monoidal categories. Macquarie math. Reports. Report No. 860081 (1986)

[K] Kac, V.G.: Infinite Dimensional Lie Algebras. Cambridge: Cambridge University Press 1990

[KaL] Kazhdan, D., Lusztig, G.: Affine Lie algebras and quantum groups. Duke Math. J. 62, 21-29, IMRN (1991)

[KaR] Kazhdan, D., Reshetikhin, N.Yu.: Balanced categories and invariants of 3-manifolds, preprint 1991

[KR1] Kirillov, A.N., Reshetikhin, N.Yu.: Representations of the algebra $U_{q}(\mathfrak{s l}(2)), q$-orthogonal polynomials and invariants of links in Infinite-dimensional Lie algebras and groups. Kac. V.G. (ed.). Singapore: World Scientific 1989

[KR2] Kirillov, A.N., Reshetikhin, N.Yu.: $q$-Weyl group and a multiplicative formula for universal $R$-matrices. Commun. Math. Phys. 134, 421-431 (1991)

[Koh] Kohno, T.: Monodromy representations of braid groups and Yang-Baxter equations. Ann. Inst. Fourier 37, 139-160 (1987)

[Koo] Koornwinder, T.H.: Representations of the twisted $S U(2)$ quantum group and some $q$-hypergeometric orthogonal polynomials. Nederl. Akad. Wetensch. Proc. Ser. A 92, 97-117 (1989)

[KZ] Knizhnik, V.G., Zamolodchikov, A.B.: Current algebra and Wess-Zumino model in two dimensions. Nucl. Phys. B 247, 83-103 (1984)

[LSo] Levendorskii, S.Z., Soibelman, Y.S.: Some applications of quantum Weyl group 1. The multiplicative formula for universal $R$-matrix for simple Lie algebras. J. Geom. Phys. 7, 1-14 (1991)

[L1] Lusztig, G.: Quantum deformations of certain simple modules over enveloping algebras. Adv. Math. 70, 237-249 (1988)

[ML] MacLane, S.: Categories for the working mathematician. Berlin, Heidelberg, New York: Springer 1988

[MMNNU] Masuda, T., Mimachi, K., Nakagami, Y., Noumi, N., Ueno, K.: Representations of the quantum group $S U_{q}(2)$ and the little $q$-Jacobi polynomials. J. Funct. Anal. 99, 357-386 (1991)

[MS] Moore, G., Seiberg, N.: Classical and quantum conformal field theory. Commun. Math. Phys. 123, 177-254 (1989)

[PS] Pressley, A., Segal, G.: Loop Groups, Oxford Mathematical Monographs, Oxford: Clarendon Press 1986

[Re1] Reshetikhin, N.Yu.: Quantized universal enveloping algebras, the Yang-Baxter equation and invariants of links, I. LOMI preprint E-4-87, 1988; II. LOMI preprint E-17-87, 1988

[Re2] Reshetikhin, N.Yu.: Quasitriangular Hopf algebras and invariants of links. Leningrad Math. J. 1, 169-188 (1989)

[RT] Reshetikhin, N.Yu., Turaev, V.G.: Ribbon graphs and their invariants derived from quantum groups. Commun. Math. Phys. 127, 1-26 (1990)

[RT1] Reshetikhin, N.Yu., Turaev, V.G.: Invariants of 3-manifolds via link polynomials and quantum groups. Invent. Math. 103, 547-597 (1991)

[Ro1] Rosso, M.: Finite dimensional representations of the quantum analogue of the enveloping algebra of a complex simple Lie algebra. Commun. Math. Phys. 117, 581-593 (1988)

[Ro2] Rosso, M.: An analogue of P.B.W. theorem and the universal $R$-matrix for $U_{h} \mathfrak{s l}(N+1)$. Commun. Math. Phys. 124, 307-318 (1989)

[S] Segal, G.: Lectures on modular functor, preprint titled "not for distribution"

[SoV] Soibelman, Ya., Vaksman, L.: Algebra of functions on the quantized group $S U(2)$. Funck. Anal. i ego Pril. 22:3, 1-14 (1988)

[SV1] Schechtman, V.V., Varchenko, A.N.: Arrangement of hyperplanes and Lie algebra homology. Invent. Math. 106. 139-194 (1991)

[SV2] Schechtman, V.V., Varchenko, A.N.: Quantum groups and homology of local systems. IAS preprint (1990) 
[TK] Tsuchiya, A., Kanie, Y.: Vertex operators in conformal field theory on $P^{1}$ and monodromy representations of braid group. Adv. Stud. Pure Math. 16, 297-372 (1988)

[W1] Witten, E.: Non-abelian bosonization in two dimensions. Commun. Math. Phys. 92, 455-472 (1984)

[W2] Witten, E.: Quantum field theory and Jones polynomial. Commun. Math. Phys. 121, 351-399 (1989)

\section{Quantum Affine Algebras and Yangians}

[BL] Bernard, D., LeClair, A.: $q$-Deformation of $S U(1,1)$ conformal Ward identities and $q$-strings. Phys. Lett. 227 B, 417-423 (1989)

[Ch1] Cherednik, I.V.: On irreducible representations of elliptic quantum $R$-algebras. DANSSSR 291, N1, 49-53 (1986)

[CP1] Chari, V., Pressley, A.: Yangians and $R$-matrices, L'Enseignement Mathématique 36, 267302 (1990)

[CP2] Chari, V., Pressley, A.: Quantum affine algebras. Commun. Math. Phys. 142, 261-283 (1991)

[CP3] Chari, V., Pressley, A.: Fundamental representations of Yangians and singularities of $R$ matrices. J. Reine Angew. Math. 417, 87-128 (1991)

[Dr1] Drinfeld, V.G.: Hopf algebras and the quantum Yang-Baxter equation. Soviet. Math. Dokl. 32, 254-258 (1985)

[Dr2] Drinfeld, V.G.: A new realization of Yangians and quantized affine algebras. Soviet. Math. Dokl. 36, 212-216 (1988)

[Dr3] Drinfeld, V.G.: Quantum groups, Proc. ICM-86 (Berkeley), Vol. 1, 798, AMS (1987)

[FJ] Frenkel, I.B., Jing, N.: Vertex representations of quantum affine algebras. Proc. Natl. Acad. Sci. USA 85, 9373-9377 (1988)

[J1] Jimbo, M.: Quantum $R$ matrix for the generalized Toda system. Commun. Math. Phys. 102, 537-547 (1986)

[J2] Jimbo, M.: A $q$-difference analogue of $U(\mathfrak{g})$ and the Yang-Baxter equation. Lett. Math. Phys. 10, 63-69 (1985)

[J3] Jimbo, M.: A $q$-analogue of $U(\mathfrak{g l}(N+1))$. Hecke algebra, and the Yang-Baxter equation. Lett. Math. Phys. 11, 247-252 (1986)

[KR] Kirillov, A.N., Reshetikhin, N.Yu.: The Yangians, Bethe ansatz and combinatorics. Lett. Math. Phys. 12, 199-208 (1986)

[RS] Reshetikhin, N.Yu., Semenov-Tian-Shansky, M.A.: Central extensions of quantum current groups. Lett. Math. Phys. 19, 133-142 (1990)

[RS1] Reshetikhin, N.Yu., Semenov-Tian-Shansky, M.A.: Factorization problem in quantum group, preprint 1991

\section{Linear Difference Equations and Basic Hypergeometric Functions}

[Ad] Adams, C.R.: On the linear ordinary $q$-difference equation. Ann. Math. 30, 195-205 (1929)

[A1] Aomoto, K.: A note on holonomic $q$-difference system. Algebraic analysis. I. Kashiwara, M., Kawai, T. (eds.). New York: Academic Press 1988, pp. 25-28

[A2] Aomoto, K.: Connection coefficients for Jackson integrals of extended Selberg type, preprint 1991

[A3] Aomoto, K.: Finiteness of a cohomology associated with certain Jackson integrals. Tôhoku Math. J. 43, 75-101 (1991)

[AW] Askey, R., Wilson, J.: Some basic orthogonal polynomials that generalize Jacobi polynomials. Mem. AMS 54, N319 (1985)

[Bi] Birkhoff, G.D.: The generalized Riemann problem for linear differential equations and the allied problems for linear difference and $q$-difference equations. Proc. Am. Acad. Arts Sci. 49, 521-568 (1913)

[C] Carmichael, R.D.: The general theory of linear $q$-difference equations. Am. J. Math. 34, 147-168 (1912)

[GR] Gasper, G., Rahman, M.: Basic hypergeometric series, Encyclopedia of Mathematics and its Applications. Cambridge: Cambridge University Press 1990 
[H1] Heine, E.: Über die Reihe .... J. Reine Angew. Math. 32, 210-212 (1846)

[H2] Heine, E.: Untersuchungen über die Reihe .... J. Reine Angew. Math. 34, 285-328 (1847)

[Ja] Jackson, F.H.: On $q$-definite integrals. Quant. J. Pure Appl. Math. 41, 193-203 (1910)

[M] Mimachi, K.: Connection problem in holonomic $q$-difference system associated with a Jackson integral of Jordan-Pochhammer type. Nagoya Math. J. 116, 149-161 (1989)

[S] Slater, L.J.: Generalized hypergeometric functions. Cambridge: Cambridge University Press 1966

[T] Trjitzinsky, W.J.: Analytic theory of linear $q$-difference equations. Acta. Math. 61, 1-38 (1933)

\section{Integrable Models in Statistical Mechanics and Quantum Field Theory}

[ABF] Andrews, G., Baxter, R., Forrester, P.: Eight-vertex SOS model and generalized RogersRamanujan-type identities. J. Stat. Phys. 35, 193-266 (1984)

[B1] Baxter, R.J.: Eight-vertex model in lattice statistics and one-dimensional anisotropic Heisenberg chain II. Equivalence to a generalized ice-type model. Ann. Phys. 76, 25-47 (1973)

[B2] Baxter, R.J.: Exactly solved models in statistical mechanics. London: Academic Press 1982

[Be] Bernard, D.: Hidden Yangians in $2 D$ massive current algebras. Commun. Math. Phys. (1992)

[Bel] Belavin, A.A.: Nucl. Phys. B 180, [FS2], 189 (19̋81)

[DJKMO] Date, E., Jimbo, M., Kuniba, A., Miwa, T., Okada, M.: Exactly solvable SOS models II: Proof of the star-triangle relation and combinatorial identities. Adv. Stud. Pure Math. vol. 16, pp. 17-22. Tokyo: Kinokuniya 1988

[DJMO] Date, E., Jimbo, M., Miwa, T., Okado, M.: Fusion of the eight-vertex SOS model. Lett. Math. Phys. 12, 209-215 (1986); Erratum and Addendum. Lett. Math. Phys. 14, 97 (1987)

[DJMO1] Date, E., Jimbo, M., Miwa, T., Okada, M.: Solvable lattice models. Proceedings Symposia Pure Mathematics 49, 295-331 (1989)

[F] Faddeev, L.D.: Integrable models in $(1+1)$-dimensional quantum field theory. Les Houche 1982, Elsevier Science Publ. 1984

[JKMO] Jimbo, M., Kuniba, A., Miwa, T., Okado, M.: The $A_{n}^{(1)}$ face models. Commun. Math. Phys. 119, 543-565 (1988)

[JMO] Jimbo, M., Miwa, T., Okado, M.: Solvable lattice models related to the vector representation of classical simple Lie algebras. Commun. Math. Phys. 116, 507-525 (1988)

[KuR] Kulish, P.P., Reshetikhin, N.Yu.: Quantum linear problem for the Since-Gordon equation and higher representations. Zap. Nauch. Semin. LOMI 101 (1981)

[KRS] Kulish, P.P., Reshetikhin, N.Yu., Sklyanin, E.K.: Yang-Baxter equation and representation theory I. Lett. Math. Phys. 5, 393-403 (1981)

[KS] Kulish, P.P., Sklyanin, E.K.: in Integrable quantum field theories. Lect. Notes in Phys. 151, 61-119. Berlin, Heidelberg, New York: Springer 1981

[LSm] LeClair, A., Smirnov, F.A.: Infinite quantum group symmetry of fields in massive $2 D$ quantum field theory, cornell preprint (1991)

[P] Pasquier, V.: Etiology of IRF models. Commun. Math. Phys. 118, 355-364 (1986)

[RS] Reshetikhin, N.Yu., Smirnov, F.: Hidden quantum group symmetry and integrable perturbations of conformal field theories. Commun. Math. Phys. 131, 157-177 (1990)

[Sk1] Sklyanin, E.K.: Some algebraic structures connected with the Yang-Baxter equation. Func. Anal. Appl. 16, 27-34 (1982)

[Sk1] Sklyanin, E.K.: Some algebraic structures connected with the Yang-Baxter equation. Representations of quantum algebras. Funct. Anal. Appl. 17, 273-284 (1983)

[Sm1] Smirnov, F.A.: Form factors in completely integrable models of quantum field theory. Singapore: World Scientific 1991

[Sm2] Smirnov, F.A.: Dynamical Symmetries of Massive Integrable Models. RIMS-772 preprint (1991)

[Z] Zamolodchikov, A.B.: Int. J. Mod. Phys. 3, 743 (1988) 